\title{
Evaluation of the effects of irradiation on bone and osteoradionecrosis of the mandible in a large animal model
}

Citation for published version (APA):

Poort, L. J. (2015). Evaluation of the effects of irradiation on bone and osteoradionecrosis of the mandible in a large animal model. [Doctoral Thesis, Maastricht University]. Datawyse / Universitaire Pers Maastricht. https://doi.org/10.26481/dis.20151210lp

Document status and date:

Published: 01/01/2015

DOI:

10.26481/dis.20151210lp

Document Version:

Publisher's PDF, also known as Version of record

\section{Please check the document version of this publication:}

- A submitted manuscript is the version of the article upon submission and before peer-review. There can be important differences between the submitted version and the official published version of record.

People interested in the research are advised to contact the author for the final version of the publication, or visit the DOI to the publisher's website.

- The final author version and the galley proof are versions of the publication after peer review.

- The final published version features the final layout of the paper including the volume, issue and page numbers.

Link to publication

\footnotetext{
General rights rights.

- You may freely distribute the URL identifying the publication in the public portal. please follow below link for the End User Agreement:

www.umlib.nl/taverne-license

Take down policy

If you believe that this document breaches copyright please contact us at:

repository@maastrichtuniversity.nl

providing details and we will investigate your claim.
}

Copyright and moral rights for the publications made accessible in the public portal are retained by the authors and/or other copyright owners and it is a condition of accessing publications that users recognise and abide by the legal requirements associated with these

- Users may download and print one copy of any publication from the public portal for the purpose of private study or research.

- You may not further distribute the material or use it for any profit-making activity or commercial gain

If the publication is distributed under the terms of Article $25 \mathrm{fa}$ of the Dutch Copyright Act, indicated by the "Taverne" license above, 
Evaluation of the effects of iriadiation on bone and osteoradjonecrosis of the mandible in a large animal model

Lucas Poort

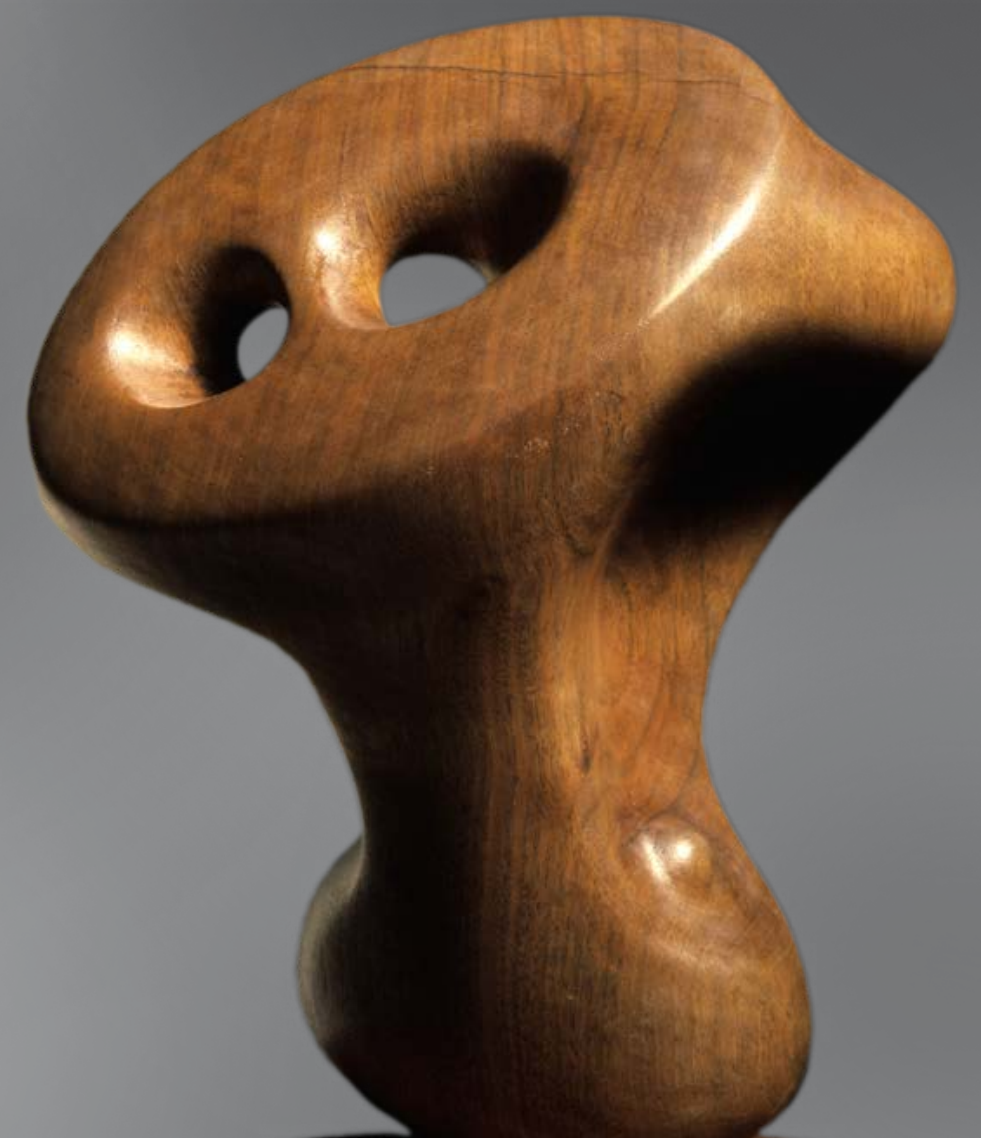


Cover illustration: Walnut wood composition 1933 Henry Moore (C) Copyright L.J. Poort

Production: Datawyse | Universitaire Pers Maastricht 


\title{
Evaluation of the effects of irradiation on bone and osteoradionecrosis of the mandible in a large animal model
}

\section{Evaluatie van bestralingseffecten op het bot en osteoradionecrose van de mandibula in een groot dier model}

\author{
PROEFSCHRIFT \\ ter verkrijging van de graad van doctor \\ aan de Universiteit Maastricht \\ op gezag van de Rector Magnificus, prof. dr. L.L.G. Soete \\ volgens het besluit van het College van Decanen, \\ in het openbaar te verdedigen \\ op donderdag 10 december 2015 om 10.00 uur
}

door

Lucas Juliën Poort

Geboren te Rotterdam, op 22 oktober 1975 
Promotor

Prof. Dr. Dr. P.A.W.H. Kessler

\section{Co-promotores}

Dr. F.J.P. Hoebers

Dr. A.A. Jacobi-Postma

\section{Beoordelingscommissie}

Prof. Dr. P. Lambin (voorzitter)

Prof. Dr. A.G. Becking, Universiteit van Amsterdam

Dr. P.J. Emans

Prof. Dr Dr. J Hoffmann, Universiteit Heidelberg

Prof. Dr J.E. Wildberger 


\section{Contents}

CHAPTER 2 Experimental studies on the irradiation of facial bones in animals: a review

CHAPTER 3 Assessment of local blood flow with laser doppler flowmetry in irradiated mandibular and frontal bone, an experiment in Göttingen minipigs.

CHAPTER 4 Does a change in bone mineral density occur in the mandible of Göttingen minipigs after irradiation in correlation with radiation dose and implant surgery?

CHAPTER 5 Radiological changes with MR-imaging and CT after irradiating minipig mandibles. The role of T2-SPIR mixed signal intensities in the detection of osteoradionecrosis.

CHAPTER 6 Dual-Energy CT versus MR imaging for the detection of bone marrow edema in pig mandibles treated with radiotherapy and surgery

CHAPTER 7 The histological and histomorphometric changes in the mandible after radiotherapy. An animal model.

CHAPTER 8 General Discussion and Future Perspectives

Valorisation addendum

SUMMARY

SAMENVATTING

Other publications

Curriculum Vitae

Dankwoord 
- aan mijn ouders - 
CHAPTER 1

General introduction 



\section{HEAD AND NECK CANCER- CLASSIFICATION, STAGING AND TREATMENT}

Head and neck cancer constitutes about $5 \%$ of all cancers in the western world. Belonging to the group of Head and Neck Cancers are malignancies from various sites within the head and neck region, including the oral cavity, the pharynx and larynx.

Cancers of the oropharynx and the oral cavity represent more than half of all head and neck cancers. ${ }^{1}$ Histopathologically, squamous cell carcinomas are by far the most common cancer type in the head and neck (head and neck squamous cell carcinomas, HNSCC). Important risk factors associated with HNSCC are tobacco and alcohol abuse as well as viral infections including human papilloma virus. ${ }^{1}$ Ageing and genetic predisposition are also important factors for the development of HNSCC. ${ }^{2}$

The mortality rates of HNSCC have shown little change over the last 30 years. Despite the improved application of multimodal therapy, patients still frequently suffer from locoregional and distant recurrences, and the survival rate of patients remain poor. This stresses the need for new tactics regarding early diagnosis and treatment alternatives. ${ }^{1,3}$

To describe the anatomic extent of a tumour in general, lymph node and distant metastasis, the TNM-classification is also used to stage HNSCC. Correspondingly for staging of HNSCC the TNM-classification is used. ${ }^{4}$ According to the sixth edition of the UICC (Union for International Cancer Control), HNSCC is then classified by - the tumour extent at the primary site ( $\mathrm{T} 1-3, \mathrm{~T} 4 \mathrm{a}, \mathrm{T} 4 \mathrm{~b})$, regional lymph node metastases (NO-3), and distant metastases (MO/M1) resulting in 40 possible combinations $(5 \times 4 \times 2)$. This offers a detailed description of the anatomic extent of the tumour which is essential for treatment planning. However, a further summarization is necessary to obtain a reasonable number of homogeneous and distinct categories with respect to survival. For that reason the TNM-classification can be transcribed into the IUC stages I, II, III, IVa, $\mathrm{IVb}$, and IVc. ${ }^{5}$ Staging the disease facilitates clinical management varying from surgery, radiotherapy $(\mathrm{RT})$, chemotherapy or a combination of the three. ${ }^{6}$

In general, early stages are managed with single modality treatment, while advanced stage disease is managed with multimodality treatment. ${ }^{7}$

Significant morbidity and poor control rates associated with primary RT to the oral cavity has led surgery to be the primary treatment modality for oral cavity carcinomas. In contrast, incremental improvement in the non-surgical management of oropharyngeal, laryngeal and hypopharyngeal cancer has led to primary RT, often combined with systemic therapy, being the preferred treatment for advanced-stage laryngeal, hypopharyngeal and oropharyngeal cancers. ${ }^{7}$

In particular patients who have advanced stage disease of the oral cavity require adjuvant treatment after surgery. Pathological findings following surgical resection determine the necessity and intensity of adjuvant treatment. In the presence of 
multiple positive nodes, perineural invasion, or angiolymphatic invasion, adjuvant RT is recommended. ${ }^{8}$

In the presence of positive surgical margins or extracapsular spread in metastatic lymph nodes adjuvant concurrent chemo-radiation is recommended. This addition has been found to diminish loco-regional recurrence and improve survival. ${ }^{9}$

\section{RADIOTHERAPY IN HEAD AND NECK CANCER}

Most head and neck cancers are treated with external beam RT, most commonly high energy photons are used. ${ }^{10}$

RT for head and neck cancer has beneficial effects on loco-regional recurrence and/or survival. $^{8,11-15}$ In addition to the beneficial effects of radiation, damage to surrounding healthy tissues may occur during and following RT. Acute side effects include, radiation dermatitis, hair loss, mucositis, taste loss, swallowing pain and salivary dysfunction. Acute side effects usually resolve over the course of several weeks following therapy, and can be managed in clinical practice by applying specific measures. However, the late side effects of radiation occur months to years after RT and are usually nonreversible, hence their prevention is very important. Late effects after RT for HNSCC include xerostomia, dental caries, fibrosis, swallowing difficulties, vascular damage and osteoradionecrosis. Radiotherapeutic techniques have improved over the years with the development of intensity-modulated RT (IMRT), which has shown a reduction in late side effects including xerostomia and dysphagia. ${ }^{16-20}$

Despite improvements, irradiation affects the surrounding healthy tissues, and can cause (late) complications. ${ }^{21-24}$ One of the most serious late complications of radiation for HNSCC is osteoradionecrosis (ORN), which can affect all bony structures in the irradiated field, and is in head and neck cancer most often seen in the mandible with a greatly varying frequency of $2-22 \% .^{25,26}$

\section{OSTEORADIONECROSIS OF THE JAWS}

The diagnosis of ORN is based on clinical presentation; "ORN is defined as a condition in which irradiated bone becomes exposed through a wound in the overlying skin or mucosa and persists without healing for three months without the recurrence of cancer". ${ }^{27}$

Several factors have been identified that increase the risk of developing ORN. Treatment related factors are the radiation dose and volume. ${ }^{25,} 27$ Patient related factors are age, gender, general health, nutritional state, continued tobacco and alcohol usage as well as the dental status. ${ }^{25}$ ORN can be triggered by surgical intervention, 
pressure sores caused by dentures or spontaneous occurrence. Recent studies reported decreased or even no ORN during follow-up after IMRT and a strict dental program. ${ }^{19,28}$ Marx differentiated ORN in early and late ORN (type I and II). Type I has a peak around one year after irradiation with both trauma induced ORN related to surgery, and spontaneous ORN related to irradiation induced tissue injury. Type II has a main peak 46 years after irradiation, again with trauma as possible trigger of ORN. ${ }^{29}$

The presentation of ORN is variable. It may remain unnoticed in the initial stage or may be diagnosed as a full blown case of ORN. The lesions progress, and patients can develop various symptoms, such as pain, dysesthesia, halitosis, dysgeusia, and food impaction in the area of exposed bone sequesters. In later stages, patients can present with fistulas from the oral mucosa or skin, extended bone necrosis, infections and pathological fractures. ${ }^{30}$

\section{CLASSIFICATIONS OF ORN}

The severity of the condition ranges from entirely asymptomatic to ORN causing severe pain, disfigurement, weight loss, and functional impairment of the jaws, seriously impairing a patient's quality of life. ${ }^{31}$

Over the years various classifications have been developed of which three gained more widespread implementation, defined by respectively Notani, Epstein and Marx. ${ }^{32,33,34}$ The Notani classification is mainly based on the anatomic boundaries of ORN and defines three categories: confined to dento-alveolar bone, limited to dento-alveolar bone or the mandible above the inferior alveolar canal, or both, and involving the mandible below the inferior alveolar canal, or pathological fracture, or skin fistula. ${ }^{32}$ Marx's classification is based on the response to hyperbaric oxygen (HBO) treatment: responsive to $\mathrm{HBO}$, unresponsive to initial $\mathrm{HBO}$ but responsive after conservative surgery and unresponsive to combination of $\mathrm{HBO}$ and conservative surgical treatment. ${ }^{34}$ Epstein's classification again includes three categories based on progression: healed, chronic but non-progressive, and active progressive (Table 1$){ }^{33}$

Table 1. Classifications of ORN

\begin{tabular}{llll}
\hline & Notani $^{32}$ & Marx $^{34}$ & Epstein $^{33}$ \\
\hline Grade I & ORN limited to dento-alveolar bone & ORN responsive to HBO treatment & Healed ORN \\
Grade II & $\begin{array}{l}\text { ORN limited to bone above inferior } \\
\text { alveolar canal }\end{array}$ & $\begin{array}{l}\text { ORN non-responsive to initial HBO } \\
\text { treatment, but responsive after } \\
\text { conservative surgery }\end{array}$ & $\begin{array}{l}\text { Chronic ORN, non- } \\
\text { progressive ORN }\end{array}$ \\
Grade III & $\begin{array}{l}\text { ORN involving bone below inferior } \\
\text { alveolar canal, fracture or fistula }\end{array}$ & $\begin{array}{l}\text { ORN non-responsive to initial HBO } \\
\text { treatment and conservative surgery }\end{array}$ & ORN \\
\hline
\end{tabular}




\section{PATHOPHYSIOLOGY OF ORN}

Regaud in 1922 was the first to describe the pathophysiology of ORN. He observed that ORN occurred in both dentulous and edentulous patients, either spontaneously or after trauma. $^{35}$

Regaud observed clinically that: "ORN differed from typical osteomyelitis in that there was no line of demarcation between the dead and viable bone. When the unrelenting process had reached the boundary of the irradiated field, the sequence of involucrum formation, sequestration, and healing proceeded in the ordinary fashion". He stated that the loss of reactivity of bone compromised by irradiation was due to the decreased vascular reserve from endothelial proliferation. ${ }^{35,36}$

In 1950, McCrorie postulated that the post-irradiation osteoporosis observed in radiological studies resulted from unopposed osteoclastic activity, since microscopically no surviving osteoblasts were seen. He stated that the lethal irradiation dose for osteoblasts was less than for osteoclasts, allowing for unopposed osteolysis in the unbalanced dynamics of bone metabolism. ${ }^{36,37}$

Marx et al. showed histologic findings of fibrosis of the mucosa, skin, and marrow spaces, hyalinization and thrombosis of vessels with loss of osteocytes and osteoblasts, and reduced vascularity of connective tissue. He described ORN as the result of a complex metabolic homeostatic deficiency of tissue that is created by radiation-induced cellular injury; "A sequence of irradiation, formation of hypoxic-, hypocellular-, and hypovascular tissue; and breakdown of tissue driven by persistent hypoxia that can cause a chronic non-healing wound". ${ }^{29}$ He observed six histological processes: hyperemia, inflammation (endarteritis), thrombosis, cellular loss, hypovascularity and fibrosis. In general hyperemia and endarteritis began early in the radiation sequence and persisted for up to 6 months after radiation treatment. Over time histology showed progressive vascular damage, endarteritis, thrombosis and fibrosis. ${ }^{29,34}$

Delanian et al. $^{38}$ stated that, radiation induced fibrosis (RIF) caused the damage in normal tissues after RT, due to the activation and dysregulation of fibroblastic activity that results in atrophic tissue. Direct and indirectly injured endothelial cells produce chemotactic cytokines that trigger acute inflammatory responses. The destruction of endothelial cells, coupled with vascular thrombosis, leads to necrosis of microvessels, local ischemia, and tissue loss. Loss of the natural endothelial cell barrier allows leakage of various cytokines that cause fibroblasts to become myofibroblast. The myofibroblasts are characterized by unusually high rates of proliferation, secretion of abnormal products of the extracellular matrix, and a reduced ability to degrade such components. $^{38}$ The mandible is predisposed to the development of ORN. This is principally the result of fibrosis that causes obliteration of the inferior alveolar artery together with the failure of the facial artery to jointly form a supply. Ultimately, the 
myofibroblasts undergo apoptosis and, even many years after RT, the bone remains, poorly vascularized, and fibrotic. ${ }^{27,30}$

Store et al. performed a micro-radiographic and histomorphometric study in osteoradionecrotic mandibular cortical bone and found, contrasting the histological findings in the bone marrow, an increase in vascular channels in the cortical bone. ${ }^{39}$ This matched clinical findings on scintigraphy scans of radionecrotic cortical bone, which showed increased uptake of radionuclides at the osteoradionecrotic bone site. ${ }^{40}$

The osteoclasts active in the cortical destruction originate from the local haematogenous bone marrow. Part of the cortical vascular increase originates from the facial artery supply. This occurs when the inferior alveolar artery is blocked, causing an increase in cortical blood flow to the bone marrow via the periosteal vascular plexus. ${ }^{41}$ This is in contrast with the opinion of Delanian et al. who stated that there is no sufficient collateral vascular supply. ${ }^{27,30}$

\section{DIAGNOSTICS}

In treatment planning and the follow-up of irradiated patients it would be of great value to be able to evaluate and monitor the bone quality after RT. This could aid treatment planning in irradiated patients related to dento-alveolar surgery, rehabilitation with dental implants or when suspicion of ORN arises. In recent studies two experimental methods for monitoring effects of irradiation on bone were provided.

\section{Laser Doppler flowmetry}

Verdonck et al. proved laser Doppler flowmetry (LDF) to be a reproducible method for assessing local blood flow in irradiated bone. ${ }^{42}$ Blood flow is progressively affected in the bone after irradiation as pointed out in the various pathophysiological theories.

LDF uses laser light which is emitted into human tissues. Laser photons are scattered, and when light hits moving blood cells it shifts in wavelength (Doppler shift) while the wavelength of light returning from static structures remains unchanged. A fiber in the probe collects the scattered light. The magnitude of the signal and the frequency changes are directly related to the relative number and velocity of blood cells in a given volume. $^{43}$

LDF directly monitors local blood flow and therefore the process of vascular impairment in irradiated bone, however since this method is invasive the surgical trauma in itself can cause disturbances and lead to development of ORN. 
Bone mineral density

Verdonck et al. used bone mineral density measurements to assess the bone quality. To this end a CT scan of a bone phantom with a known mineral density was used. With the known density of the phantom a value related to the absolute bone mineral density was calculated. They observed a non-significant increase in bone mineral density after irradiation. ${ }^{44}$

A specific experimental method to measure bone quality and ORN non-invasively is lacking.

\section{RADIOLOGICAL IMAGING}

In the radiological diagnosis of ORN, CT and MR-imaging are complementary. CT findings in ORN are cortical disruption, with disorganization and loss of trabeculation of the mandibular spongiosa. Cortical bone fragmentation is present in most cases. ${ }^{45}$ More severe cases often present with a pathologic fracture. The abnormalities are predominantly seen in the body of the mandible (premolar and molar region), in some cases they can extend into the angular region or retromolar triangle. ${ }^{45}$

Generally MR-imaging of mandibular ORN reveals tissue reaction, edema, mimicking "inflammation" that diffusely affects the bone and bone marrow. Edema of the marrow is visualized on MR-imaging by reduced signal intensity on T1-weighted images and increased signal intensity on T2-weighted images. However only few studies describe imaging of ORN in the mandible and little is known about imaging of short and longterm irradiation effects of the mandible so far. ${ }^{46}$

\section{TREATMENT OF ORN}

\section{Conservative treatment}

Post-irradiation bone lesions may be managed with conservative and surgical modalities. Conservative therapies include multiple daily saline irrigations, antibiotics during infectious episodes, topically applied antiseptics, gentle sequestrectomy with removal of visibly loosened bone fragments. ${ }^{26}$ In the conservative management, local irritants such as alcohol, tobacco, smoking, and ill-fitting dentures must be avoided. ${ }^{26}$

\section{Surgical treatment}

If the treatment of ORN with conservative management, with or without surgical debridement fails, resections of various extents are indicated. Due to the compromised 
local tissue condition, reconstruction with micro-surgical free-flap transfer is the standard treatment in extended necrotic destruction. Mandibular reconstruction with a micro-surgical free fibula flap is an elegant, but extensive and invasive solution to restore the anatomic arch, oral functions, and facial aesthetics. Dental prosthetic stabilization is complicated by the replaced thin cutaneous layer together with relative thick subcutaneous tissues below and the absence of a lingual and vestibular groove. However dental implants can improve restored prosthetic functionality. ${ }^{26,47}$

To point out the difficulties of treatment of ORN Notani et al. reported the outcome of conservative and surgical treatment of ORN in a small patient cohort. They found that patients suffering from grade I ORN treated conservatively fully recovered ( 2 of 2 cases). However, in grade II cases, only 69\% recuperated after conservative treatment (18 of 26 cases) and $88 \%$ needed sequestrectomy (7 of 8 cases). In patients suffering from grade III ORN, only $10 \%$ improved with conservative treatment ( 3 of 30 cases), no patient took advantage of sequestrectomy ( 0 of 6 cases) and $87 \%$ only healed after partial or complete mandibulectomy (13 of 15 cases). Of all 87 ORN patients, 15 (17\%) had to undergo partial or complete mandibulectomy. ${ }^{32}$

\section{Medical treatment of ORN}

The medical treatment of ORN consists of a combination therapy of pentoxifilline, tocopherol and clodronate (PENTOCLO) developed by Delanian et al. ${ }^{38}$ The combination therapy works on radiation-induced ORN and targets radiation-induced bone fibrosis and stimulates osteogenesis via the antioxidant pathway. ${ }^{38}$ Pentoxifilline increases the level of oxygenation in the tissues and vitamin $E$ has an antioxidant action. The Pentoxifilline-vitamin E combination was shown to significantly decreases radiationinduced fibrosis via a synergistic action in a randomised clinical trial in humans. ${ }^{38,48}$ For the treatment of ORN in the mandible the addition of Clodronate inhibits osteoclastic bone destruction. ${ }^{49,50}$ Unlike other bisfosfonates Clodronate also acts on osteoblasts by increasing formation of bone and reducing proliferation of fibroblasts. ${ }^{51}$

Complete recovery of ORN in a median of 9 months is reported in a phase II trial conducted by Delanian et al. ${ }^{52} \mathrm{~A}$ recent retrospective study on PENTOCLO treatment of ORN reported healing of 16 out of 21 ORN patients in 6 months treatment. ${ }^{50}$ Long-term PENTOCLO treatment is effective, safe, and curative for refractory ORN and induces mucosal and bone healing with significant symptom improvement. However the majority of these patients were included with Marx grade I ORN and some had grade II. No patients had clinical grade III ORN. PENTOCLO has not been shown to improve severe cases of ORN and for these cases early intervention can be the better choice, as advocated by Jacobson et al. ${ }^{47}$ 


\section{Treatment of ORN with $\mathrm{HBO}$}

HBO therapy is a well-known treatment therapy for ORN since 1976, after it was first introduced by Hart et al. ${ }^{53}$ The rationale behind the use of HBO therapy lies in the fact that the delivery of oxygen is an essential process when considering bone tissue repair. Inadequate vascularity of bone reduces osteogenesis. Oxygen stimulates collagen synthesis, matrix deposition, angiogenesis, epithelialization, and the eradication of bacteria. The use of HBO therapy in improving wound healing is based on this principle. The long-term effects are neovascularisation, osteogenesis, and a stimulation of collagen production by fibroblast, all of which promote wound healing. ${ }^{54}$

The current protocol for HBO therapy is 20-30 dives at 2-2.5 atmospheres for 90$120 \mathrm{~min}$ at each session.

There are various studies on $\mathrm{HBO}$ in the management of ORN, but recent studies have questioned its effectiveness.

A classical study was done by Marx et al. ${ }^{55}$, which advocates the use of HBO in the treatment of ORN. He has reported the successful treatment of 268 ORN cases, in a retrospective study, these cases were grade I and II from the Marx classification. ${ }^{56} \mathrm{~A}$ randomized placebo-controlled multicenter study found, however, no difference between conventional conservative treatment and HBO treatment for ORN. They also found no benefit from HBO in patients with overt mandibular ORN. This study excluded patients with pathological fractures and inferior mandibular border involvement at moment of inclusion, including only grade I and II cases from the Notani classification. ${ }^{56}$ A Cochrane review has been done and concluded that there is some evidence that HBO improves outcome in late irradiation tissue injury that affects bone and soft tissues in the head and neck region. ${ }^{57}$

\section{CONCLUSION}

ORN is a serious condition that can develop at any time after irradiation in the head and neck region. Despite some outspoken theories on pathophysiology of irradiation effects on all tissues, but especially bone and the development of ORN, it is unclear why some patients develop ORN after radiotherapy to the head and neck and some do not. Experimental evidence on irradiation effects and ORN is multi-fold, however interpretation of these studies and its clinical implications remains difficult. As there are only few studies on imaging of post-irradiation effects and ORN, this thesis also aims at the description of image based characteristics of typical early morphological changes in the mandible in a large-animal experiment. 


\section{OBJECTIVES OF THIS THESIS}

The aim of this thesis was to investigate the effects of irradiation on the mandible by

1. reviewing animal experiments on irradiation in the head and neck

2. assessment of mandibular vascularisation after irradiation compared to the frontal bone

3. assessment of mandibular bone mineral density changes after irradiation with various doses

4. identification of specific post-RT imaging patterns in MR- and CT-scans

5. validation of the Dual Energy $\mathrm{CT}$ as a diagnostic tool for irradiation induced mandibular bone marrow edema

6. identification of RT-induced histological effects to support the clinical and diagnostic findings

\section{OUTLINE OF THIS THESIS}

In chapter 2 a literature review is presented relevant for this study. The review focuses on animal-based study models on ORN. This information led to the development of a new animal model on irradiation side-effects in the head and neck region.

We evaluated the effects of irradiation on healthy mandibular bone in our large animal model. In chapter 3 we measured the effect of irradiation on bone vascularity with laser doppler flowmetry in correlation to radiation dose.

In chapter 4 we analysed bone mineral density changes in correlation to radiation dose. We studied imaging characteristics of radiation effects and of osteoradionecrosis with both MR-imaging and CT after irradiation with various doses in chapter 5.

In chapter 6 Dual Energy Computer Tomography was tested as alternative modality to MR-imaging for detection of bone marrow edema in the mandible.

In chapter 7 we looked at histological and histomorphometric changes after irradiation in our large animal model

In chapter 8 the results and impact of the studies presented in this thesis were discussed. 


\section{REFERENCES}

1. Curado MP and Hashibe M, Recent changes in the epidemiology of head and neck cancer. Curr Opin Oncol, 2009. 21(3): p. 194-200.

2. Riaz N, Morris LG, Lee $W$ and Chan TA, Unraveling the molecular genetics of head and neck cancer through genome-wide approaches. Genes Dis, 2014. 1(1): p. 75-86.

3. Jemal A, Bray F, Center MM, Ferlay J, Ward E and Forman D, Global cancer statistics. CA Cancer J Clin, 2011. 61(2): p. 69-90.

4. Gregoire V, Lefebvre JL, Licitra L and Felip E, Squamous cell carcinoma of the head and neck: EHNSESMO-ESTRO Clinical Practice Guidelines for diagnosis, treatment and follow-up. Ann Oncol, 2010. 21 Suppl 5: p. v184-6.

5. Kreppel M, Eich HT, Kubler A, Zoller JE and Scheer M, Prognostic value of the sixth edition of the UICC's TNM classification and stage grouping for oral cancer. J Surg Oncol, 2010. 102(5): p. 443-9.

6. Seiwert TY, Salama JK and Vokes EE, The chemoradiation paradigm in head and neck cancer. Nat Clin Pract Oncol, 2007. 4(3): p. 156-71.

7. Lango MN, Multimodal treatment for head and neck cancer. Surg Clin North Am, 2009. 89(1): p. 43-52, viii.

8. Peters LJ, Goepfert H, Ang KK, Byers RM, Maor MH, Guillamondegui O, Morrison WH, Weber RS, Garden AS, Frankenthaler RA, and et al., Evaluation of the dose for postoperative radiation therapy of head and neck cancer: first report of a prospective randomized trial. Int J Radiat Oncol Biol Phys, 1993. 26(1): p. 311.

9. Bernier J, Cooper JS, Pajak TF, van Glabbeke M, Bourhis J, Forastiere A, Ozsahin EM, Jacobs JR, Jassem J, Ang KK, and Lefebvre JL, Defining risk levels in locally advanced head and neck cancers: a comparative analysis of concurrent postoperative radiation plus chemotherapy trials of the EORTC (\#22931) and RTOG (\# 9501). Head Neck, 2005. 27(10): p. 843-50.

10. Haraa WK, SA, General principles of radiation therapy for head and neck cancer. www.uptodate.com, 2015.

11. Klug C, Wutzl A, Kermer C, Voracek M, Kornek G, Selzer E, Glaser C, Poeschl PW, Millesi W and Ewers R, Preoperative radiochemotherapy and radical resection for stages II-IV oral and oropharyngeal cancer: outcome of 222 patients. Int J Oral Maxillofac Surg, 2005. 34(2): p. 143-8.

12. Paximadis PA, Christensen ME, Dyson G, Kamdar DP, Sukari A, Lin HS, Yoo GH and Kim HE, Up-front neck dissection followed by concurrent chemoradiation in patients with regionally advanced head and neck cancer. Head Neck, 2012.

13. Ramaekers BL, Pijls-Johannesma M, Joore MA, van den Ende $P$, Langendijk JA, Lambin P, Kessels AG and Grutters JP, Systematic review and meta-analysis of radiotherapy in various head and neck cancers: comparing photons, carbon-ions and protons. Cancer Treat Rev, 2011. 37(3): p. 185-201.

14. Egelmeer AG, Velazquez ER, de Jong JM, Oberije C, Geussens Y, Nuyts S, Kremer B, Rietveld D, Leemans CR, de Jong MC, Rasch C, Hoebers F, Homer J, Slevin N, West C, and Lambin P, Development and validation of a nomogram for prediction of survival and local control in laryngeal carcinoma patients treated with radiotherapy alone: a cohort study based on 994 patients. Radiother Oncol, 2011. 100(1): p. 108-15.

15. Bourhis J, Overgaard J, Audry H, Ang KK, Saunders M, Bernier J, Horiot JC, Le Maitre A, Pajak TF, Poulsen MG, O'Sullivan B, Dobrowsky W, Hliniak A, Skladowski K, Hay JH, Pinto LH, Fallai C, Fu KK, Sylvester R, and Pignon JP, Hyperfractionated or accelerated radiotherapy in head and neck cancer: a meta-analysis. Lancet, 2006. 368(9538): p. 843-54.

16. Studer G, Studer SP, Zwahlen RA, Huguenin P, Gratz KW, Lutolf UM and Glanzmann C, Osteoradionecrosis of the mandible: minimized risk profile following intensity-modulated radiation therapy (IMRT). Strahlenther Onkol, 2006. 182(5): p. 283-8. 
17. Ramaekers BL, Joore MA, Grutters JP, van den Ende $\mathrm{P}$, Jong J, Houben R, Lambin $\mathrm{P}$, Christianen $\mathrm{M}$, Beetz I, Pijls-Johannesma M, and Langendijk JA, The impact of late treatment-toxicity on generic health-related quality of life in head and neck cancer patients after radiotherapy. Oral Oncol, 2011. 47(8): p. 768-74.

18. Christianen ME, Langendijk JA, Westerlaan HE, van de Water TA and Bijl HP, Delineation of organs at risk involved in swallowing for radiotherapy treatment planning. Radiother Oncol, 2011. 101(3): p. 394-402.

19. Ben-David MA, Diamante M, Radawski JD, Vineberg KA, Stroup C, Murdoch-Kinch CA, Zwetchkenbaum SR and Eisbruch A, Lack of osteoradionecrosis of the mandible after intensity-modulated radiotherapy for head and neck cancer: likely contributions of both dental care and improved dose distributions. Int J Radiat Oncol Biol Phys, 2007. 68(2): p. 396-402.

20. Overgaard J, Hypoxic modification of radiotherapy in squamous cell carcinoma of the head and neck--a systematic review and meta-analysis. Radiother Oncol, 2011. 100(1): p. 22-32.

21. Lee $\mathrm{J}$ and Moon C, Current status of experimental therapeutics for head and neck cancer. Exp Biol Med (Maywood), 2011. 236(4): p. 375-89.

22. Thariat J, Bolle S, Demizu Y, Marcy PY, Hu Y, Santini J, Bourhis J and Pommier P, New techniques in radiation therapy for head and neck cancer: IMRT, CyberKnife, protons, and carbon ions, Improved effectiveness and safety? Impact on survival? Anticancer Drugs, 2011. 22: p. 596-606.

23. Mul VE, de Jong JM, Murrer LH, van den Ende PL, Houben RM, Lacko M, Lambin P and Baumert BG, Lhermitte sign and myelopathy after irradiation of the cervical spinal cord in radiotherapy treatment of head and neck cancer. Strahlenther Onkol, 2012. 188(1): p. 71-6.

24. Boomsma MJ, Bijl HP and Langendijk JA, Radiation-induced hypothyroidism in head and neck cancer patients: a systematic review. Radiother Oncol, 2011. 99(1): p. 1-5.

25. Teng MS and Futran ND, Osteoradionecrosis of the mandible. Curr Opin Otolaryngol Head Neck Surg, 2005. 13(4): p. 217-21.

26. Jereczek-Fossa BA and Orecchia R, Radiotherapy-induced mandibular bone complications. Cancer Treat Rev, 2002. 28(1): p. 65-74.

27. Lyons A and Ghazali N, Osteoradionecrosis of the jaws: current understanding of its pathophysiology and treatment. Br J Oral Maxillofac Surg, 2008. 46(8): p. 653-60.

28. Gomez DR, Estilo CL, Wolden SL, Zelefsky MJ, Kraus DH, Wong RJ, Shaha AR, Shah JP, Mechalakos JG and Lee NY, Correlation of osteoradionecrosis and dental events with dosimetric parameters in intensitymodulated radiation therapy for head-and-neck cancer. Int J Radiat Oncol Biol Phys. 81(4): p. e207-13.

29. Marx RE and Johnson RP, Studies in the radiobiology of osteoradionecrosis and their clinical significance. Oral Surg Oral Med Oral Pathol, 1987. 64(4): p. 379-90.

30. Zhuang $\mathrm{Q}$, Zhang $\mathrm{Z}, \mathrm{Fu} \mathrm{H}$, He J and $\mathrm{He} \mathrm{Y}$, Does radiation-induced fibrosis have an important role in pathophysiology of the osteoradionecrosis of jaw? Med Hypotheses, 2011. 77(1): p. 63-5.

31. Lyons A, Osher J, Warner E, Kumar R and Brennan PA, Osteoradionecrosis--a review of current concepts in defining the extent of the disease and a new classification proposal. Br J Oral Maxillofac Surg, 2014. 52(5): p. 392-5.

32. Notani K, Yamazaki Y, Kitada H, Sakakibara N, Fukuda H, Omori K and Nakamura M, Management of mandibular osteoradionecrosis corresponding to the severity of osteoradionecrosis and the method of radiotherapy. Head Neck, 2003. 25(3): p. 181-6.

33. Epstein JB, Wong FL and Stevenson-Moore P, Osteoradionecrosis: clinical experience and a proposal for classification. J Oral Maxillofac Surg, 1987. 45(2): p. 104-10.

34. Marx RE, Osteoradionecrosis: a new concept of its pathophysiology. J Oral Maxillofac Surg, 1983. 41(5): p. 283-8.

35. Regaud C, Sur la sensibilite du tissue osseux normale vis-a-vis les radiations $X$ et sur le mecanisme de I'osteoradionecrose. Comp Rendu Sot Biol Paris, 1922. 87(14): p. 629.

36. Wildermuth $\mathrm{O}$ and Cantril ST, Radiation necrosis of the mandible. Radiology, 1953. 61(5): p. 771-85.

37. McCrorie WD, Fractures of the femoral neck following pelvic irradiation; a review of ten cases. $\mathrm{Br} \mathrm{J}$ Radiol, 1950. 23(274): p. 587-92.

38. Delanian $\mathrm{S}$ and Lefaix $\mathrm{JL}$, The radiation-induced fibroatrophic process: therapeutic perspective via the antioxidant pathway. Radiother Oncol, 2004. 73(2): p. 119-31. 
39. Store $\mathrm{G}$ and Granstrom G, Osteoradionecrosis of the mandible: a microradiographic study of cortical bone. Scand J Plast Reconstr Surg Hand Surg, 1999. 33(3): p. 307-14.

40. Hutchison IL, Cullum ID, Langford JA, Jarritt PH, Ell PJ and Harris M, The investigation of osteoradionecrosis of the mandible by $99 \mathrm{mTc}$-methylene diphosphonate radionuclide bone scans. $\mathrm{Br} \mathrm{J}$ Oral Maxillofac Surg, 1990. 28(3): p. 143-9.

41. Hellem $\mathrm{S}$ and Ostrup LT, Normal and retrograde blood supply to the body of the mandible in the dog. II. The role played by periosteo-medullary and symphyseal anastomoses. Int J Oral Surg, 1981. 10(1): p. 3142.

42. Verdonck HW, Meijer GJ, Laurin T, Nieman FH, Stoll C, Riediger D, Stoelinga PJ and de Baat C, Assessment of vascularity in irradiated and nonirradiated maxillary and mandibular minipig alveolar bone using laser doppler flowmetry. Int J Oral Maxillofac Implants, 2007. 22(5): p. 774-8.

43. Michelson G, Schmauss B, Langhans MJ, Harazny J and Groh MJ, Principle, validity, and reliability of scanning laser Doppler flowmetry. J Glaucoma, 1996. 5(2): p. 99-105.

44. Verdonck HW, Meijer GJ, Nieman FH, Stoll C, Riediger D and de Baat C, Quantitative computed tomography bone mineral density measurements in irradiated and non-irradiated minipig alveolar bone: an experimental study. Clin Oral Implants Res, 2008. 19(5): p. 465-8.

45. Hermans R, Fossion E, loannides C, Van den Bogaert W, Ghekiere J and Baert AL, CT findings in osteoradionecrosis of the mandible. Skeletal Radiol, 1996. 25(1): p. 31-6.

46. Bachmann G, Rossler R, Klett R, Rau WS and Bauer R, The role of magnetic resonance imaging and scintigraphy in the diagnosis of pathologic changes of the mandible after radiation therapy. Int J Oral Maxillofac Surg, 1996. 25(3): p. 189-95.

47. Jacobson AS, Buchbinder D, Hu K and Urken ML, Paradigm shifts in the management of osteoradionecrosis of the mandible. Oral Oncol, 2010. 46(11): p. 795-801.

48. Delanian S, Porcher R, Balla-Mekias S and Lefaix JL, Randomized, placebo-controlled trial of combined pentoxifylline and tocopherol for regression of superficial radiation-induced fibrosis. J Clin Oncol, 2003. 21(13): p. 2545-50.

49. Delanian $\mathrm{S}$ and Lefaix JL, Complete healing of severe osteoradionecrosis with treatment combining pentoxifylline, tocopherol and clodronate. Br J Radiol, 2002. 75(893): p. 467-9.

50. Robard L, Louis MY, Blanchard D, Babin E and Delanian S, Medical treatment of osteoradionecrosis of the mandible by PENTOCLO: Preliminary results. Eur Ann Otorhinolaryngol Head Neck Dis, 2014.

51. Fast DK, Felix R, Dowse C, Neuman WF and Fleisch $H$, The effects of diphosphonates on the growth and glycolysis of connective-tissue cells in culture. Biochem J, 1978. 172(1): p. 97-107.

52. Delanian S, Chatel C, Porcher R, Depondt J and Lefaix JL, Complete Restoration of Refractory Mandibular Osteoradionecrosis by Prolonged Treatment with a Pentoxifylline-Tocopherol-Clodronate Combination (PENTOCLO): A Phase II Trial. Int J Radiat Oncol Biol Phys.

53. Hart GB and Mainous EG, The treatment of radiation necrosis with hyperbaric oxygen (OHP). Cancer, 1976. 37(6): p. 2580-5

54. Spiegelberg L, Djasim UM, van Neck HW, Wolvius EB and van der Wal KG, Hyperbaric oxygen therapy in the management of radiation-induced injury in the head and neck region: a review of the literature. J Oral Maxillofac Surg, 2010. 68(8): p. 1732-9.

55. Shaw RJ and Butterworth C, Hyperbaric oxygen in the management of late radiation injury to the head and neck. Part II: prevention. Br J Oral Maxillofac Surg, 2011. 49(1): p. 9-13.

56. Annane D, Depondt J, Aubert P, Villart M, Gehanno P, Gajdos P and Chevret S, Hyperbaric oxygen therapy for radionecrosis of the jaw: a randomized, placebo-controlled, double-blind trial from the ORN96 study group. J Clin Oncol, 2004. 22(24): p. 4893-900.

57. Bennett MH, Feldmeier J, Hampson N, Smee R and Milross C, Hyperbaric oxygen therapy for late radiation tissue injury. Cochrane Database Syst Rev, 2012. 5: p. CD005005. 


\section{CHAPTER 2}

\section{Experimental studies on the irradiation of}

facial bones in animals: a review

L.J. Poort, B. Lethaus, R.A. Böckmann, D. Buurman, J. de Jong, F.J.P. Hoebers, P.A.W.H. Kessler

Published in the International Journal of Otolaryngology and Head \& Neck Surgery, 2014, 3, 9-13 


\section{ABSTRACT}

Introduction Radiotherapy is often used to treat head and neck malignancies, with inevitable effects on the surrounding healthy tissues. We have reviewed the literature concerning the experimental irradiation of facial bones in animals.

Materials and methods A PubMed search was performed to retrieve animal experiments on the irradiation of facial bones that were published between January 1992 and January 2012. The search terms were "irradiation facial bone" and "irradiation osteoradionecrosis".

Results Thirty-six publications were included. The irradiation sources were Cobalt ${ }^{60}$, orthovoltage, 4-6 megavolt photons, and brachytherapy. The total dose varied between 8-60 Gy in single or multiple fractions. The literature presents a broad range of animal studies that differ in terms of the in vivo model, irradiation, observation period, and evaluation of results.

Discussion The different animal models used leave many questions unanswered. A detailed and standardized description of the methodology and results would facilitate the comparability of future studies. 


\section{INTRODUCTION}

For head and neck malignancies, current treatment regimes frequently include (chemo-) radiotherapy. Several clinical studies have shown the beneficial effects of radiotherapy as a primary, definitive treatment or when used in a postoperative setting after surgical resection, with respect to the time to recurrence or survival. ${ }^{1-4}$ However, despite improved radiotherapeutic techniques such as intensity-modulated radiation therapy $\left(\right.$ IMRT), ${ }^{5-9}$ irradiation effects on the surrounding healthy tissue continue to cause complications of different severities. ${ }^{10-13}$

One complication is osteoradionecrosis (ORN), which can affect all bony structures in the head and neck region but is most commonly seen in the mandible, at a frequency of $2-22 \%{ }^{14,15}$ The diagnosis is generally based on clinical presentation. ORN is defined as "irradiated bone, which becomes devitalized and is exposed through the overlying skin or mucosa and does not heal within a period of three months, without tumor recurrence". ${ }^{16}$ Several factors have been identified that increase the risk of developing ORN, including treatment-related factors such as the radiation dose and volume of irradiation. ${ }^{14,16}$ ORN can be triggered by surgical intervention or pressure sores from dentures or can occur spontaneously. It can arise at any point after irradiation therapy, even several years later. ${ }^{17}$

The treatment of ORN ranges from conservative management, with or without surgical debridement, to broad resection in severe cases. Due to the compromised local tissue condition, this treatment is often followed by reconstruction with free microsurgical flap surgery, which is a major surgical intervention. ${ }^{18-20}$

The pathophysiology of ORN and the effect of irradiation on bones were extensively described by Marx as a complex metabolic homeostatic deficiency of tissue that is created by radiation-induced cellular injury. Marx hypothesizes that a sequence of irradiation, formation of hypoxic-hypocellular-hypovascular tissue, and breakdown of tissue driven by persistent hypoxia can cause a chronic non-healing wound. ${ }^{16,17}$ Histological evaluations show progressive vascular damage that causes endarteritis, thrombosis, and fibrosis. ${ }^{17,21,22}$

In addition, Delanian et al. state that radiation-induced fibrosis accounts for the damage observed in normal tissues, including bone, after radiotherapy. The key event in this type of damage consists of the activation and deregulation of fibroblastic activity, which leads to the formation of atrophic tissue. The destruction of endothelial cells coupled with vascular thrombosis leads to the necrosis of microvessels, local ischemia, and tissue loss. ${ }^{16}$ 
The irradiated bone remain fragile and may be subject to surges of late reactivated inflammation after a physicochemical trauma, resulting in a tendency to develop ORN. The mandible is thought to be predisposed to the development of ORN principally as the result of the fibrosis, which causes the obliteration of the inferior alveolar artery and the inability of the facial artery to act as an alternative blood supply. ${ }^{16,23}$

The above-mentioned hypotheses on the pathogenesis of structural changes in bone after irradiation are mainly based on human studies. Definite answers to many questions, however, remain elusive such as the following: How much irradiation is tolerated before necrosis develops? Is there a maximum radiation dose and an optimum time frame to ensure a successful surgical intervention? What is the fractionation sensitivity (in other words, the alpha/beta ratio) of the bone? Are there some preventive measures that can be taken to prevent ORN? What is the clinical relevance of a radiologic diagnosis of ORN? Does teeth extraction before treatment increase or decrease the risk of ORN? In an effort to better understand the biological changes that occur in facial bones after irradiation, we have reviewed studies investigating the irradiation of facial bones in animal models.

We found that the literature presents a broad variety of animal studies that differ in terms of the animal model, applied dose, radiation source, observation period, and evaluation of results. We made an effort to evaluate all these models and determine whether they answer any of the questions given above. Furthermore, we aim to make a recommendation on how to optimize future studies to increase translational value and comparability.

\section{MATERIALS AND METHODS}

A PubMed search was performed for animal studies that involved the irradiation of facial bones and were published between January 1992 and January 2012. The search terms used were "irradiation facial bone" and "irradiation osteoradionecrosis," and the limit activation term was "animal".

The year of publication, experimental setup, irradiation source, irradiation dose, irradiation fractionation, kind and number of animals used, type of intervention, and radiological and histological examinations were recorded. Only studies for which the full-text publications were available were included. References of the articles found were checked regarding importance and obtained when useful.

Research questions were: what animal model was used? what irradiation scheme was used? what intervention was studied? what was the method of evaluation? what were the results? and what is the clinical implication? 


\section{RESULTS}

The search resulted in 152 publications, which included reviews and case reports. Case reports were discarded. Based on the abstracts, we reviewed the full text of 48 potentially relevant publications and included 36 publications for this review (Appendix 1). The flow chart of the selection is given in figure 1.

\section{Literature search \\ Database: PubMed \\ Limit activation: Animal \\ Period: January 1992- \\ January 2012 \\ 152 records identified}

no additional records identified through other sources

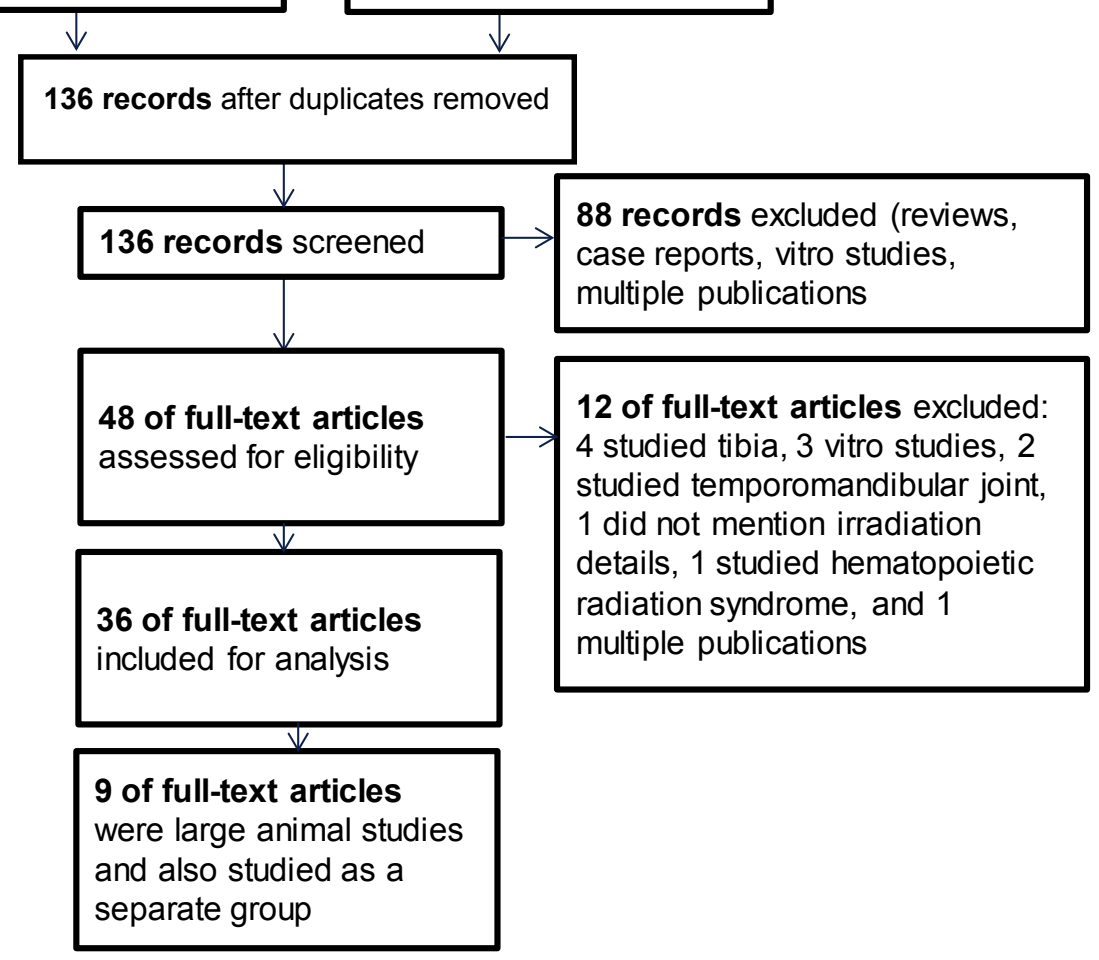

Figure 1. The flow diagram of all studies considered, excluded and included.

The publications that were excluded lacked radiological and histological evaluations of the irradiation or involved only in vitro cell cultures. The irradiation details of the included studies are shown in table 1. 
Table 1. Irradiation details of the included studies

\begin{tabular}{|c|c|c|c|c|c|c|c|}
\hline \multirow{2}{*}{$\begin{array}{l}\text { Study } \\
1\end{array}$} & \multirow{2}{*}{$\begin{array}{l}\text { Fraction } \\
\text { Dose (Gray) } \\
8\end{array}$} & \multicolumn{2}{|c|}{ Frequencylnterval } & \multirow{2}{*}{$\begin{array}{l}\text { Total Dose } \\
\text { (Gray) } \\
24\end{array}$} & \multirow{2}{*}{$\begin{array}{l}\text { Equivalent } \\
\text { Dose (Gray) } \\
64^{* *}\end{array}$} & \multirow{2}{*}{$\begin{array}{l}\text { Source } \\
\text { Co60 }\end{array}$} & \multirow{2}{*}{$\begin{array}{l}\text { Animal } \\
\text { Minipig }\end{array}$} \\
\hline & & 3 & $1 w$ & & & & \\
\hline 2 & $5,9 / 7 / 8,89$ & 5 & $1 d$ & $29,5 / 35 / 44$ & $550 / 64 / 96 * *$ & Ortho & Rat \\
\hline 3 & $5,9 / 7 / 8,89$ & 5 & $1 d$ & 29,5/35/44. & $550 / 64 / 96 * *$ & Ortho & Rat \\
\hline 4 & 30 & 1 & & 30 & & Brachy & Rat \\
\hline 5 & 30 & 1 & & 30 & $18 \mathrm{MV}$ & Rabbit & \\
\hline 6 & $6,5-9$ & 5 & $1 w$ & $32,5-45.4$ & $60-108$ & $18 \mathrm{MV}$ & Rabbit \\
\hline 7 & 3,6 & 10 & $1 d$ & 36 & & Ortho & Rat \\
\hline 8 & 8 & 1 & & 8 & & Co60 & Rat \\
\hline 9 & 3,6 & 10 & & $1 d$ & 36 & Ortho & Rat \\
\hline 10 & 5,7 & 4 & $\mathrm{~nm}$ & 22,8 & 50 & Co60 & Dog \\
\hline 11 & 15 & 1 & & 15 & 46 & Co60 & Rabbit \\
\hline 12 & $15 / 25 / 35$ & 1 & & $15 / 25 / 35$ & & Ortho & Rabbit \\
\hline 13 & 30 & 1 & & 30 & & Ortho & Rabbit \\
\hline 14 & $4 \& 5$ & $10 \& 15$ & $1 d^{*}$ & $40 / 50 / 60$ & & $6 \mathrm{MV}$ & Dog \\
\hline 15 & 4,3 & 10 & $1 d$ & 43 & & Co60 & Dog \\
\hline 16 & 5,6 & 4 & $2 x / w$ & 22,4 & 50 & $4 \mathrm{MV}$ & Rabbit \\
\hline 17 & 5,6 & 4 & $2 x / w$ & 22,4 & 50 & $4 \mathrm{MV}$ & Rabbit \\
\hline 18 & 5,6 & 4 & $2 x / w$ & 22,4 & 50 & $4 \mathrm{MV}$ & Rabbit \\
\hline 19 & 11.96 & 6 & $3 x / w$ & 71,4 & & Ortho & Rabbit \\
\hline 20 & 3 & 15 & $2-3 d$ & 45 & 50 & $6 \mathrm{MV}$ & Sheep \\
\hline 21 & 5,4 & 5 & $2 d$ & 27 & 50 & $6 \mathrm{MV}$ & Rabbit \\
\hline 22 & 5,5 & 5 & $1 w$ & 27,5 & & $6 \mathrm{MV}$ & Rabbit \\
\hline 23 & 20 & 1 & & 20 & 45 & Brachy & Rat \\
\hline 24 & 20 & 1 & & 20 & 45 & Brachy & Rat \\
\hline 25 & 15 & 4 & $2 w$ & 60 & $6 \mathrm{MV}$ & & Rat \\
\hline 26 & 6 & 7 & $2-3 d$ & 42 & & Ortho & Rat \\
\hline 27 & 4,3 & 10 & $1 d$ & 43 & & Co60 & Dog \\
\hline 28 & 2,5 & 20 & $1 d$ & 50 & & Co60 & Dog \\
\hline 29 & 4,3 & 10 & $1 d$ & 43 & 60 & Co60 & Dog \\
\hline 30 & 15 & 1 & & 15 & & Co60 & Rabbit \\
\hline 31 & 2 & 16 & $4 x / w$ & 32 & & Ortho & Rabbit \\
\hline 32 & 4 & 10 & $3 x / w$ & 40 & & Co60 & Dog \\
\hline 33 & $2,5 / 3$ & $18 / 15$ & $\mathrm{~nm}$ & 45 & & Co60 & Rat \\
\hline 34 & 2 & 27 & $1 d$ & 54 & 54 & $\mathrm{~nm}$ & Dog \\
\hline 35 & 20 & 1 & 20 & & & Brachy & Rat \\
\hline 36 & 20 & 1 & 20 & & & Ortho & Rat \\
\hline
\end{tabular}

( $d$ = days, $\mathrm{w}=$ weeks, $\mathrm{x} / \mathrm{w}=$ times per week, ortho = orthovolt, $\mathrm{MV}=$ Megavolt, Brachy = Brachytherapy, $\mathrm{nm}=$ not mentioned, ${ }^{*}=$ one week daily, one month interval for two or three weeks, and ** = calculated from $75 \%$, $100 \%, 150 \%$ assuming $100 \%$ to be 64 Gray) 


\section{Animal species, age, and gender}

The animals that were used in the studies are summarized in table 2 . The animals were exclusively adult in 21 studies (58\%) and immature animals in nine studies. In the remaining six studies, age was not specified. Male animals were used in 11 studies, and female animals were used in four studies. The majority of the studies did not specify the gender of the animals.

Table 2. Animal species used in the included studies

\begin{tabular}{lll}
\hline Animal & Number of studies & Percentage \\
\hline Rat & 13 & $36 \%$ \\
Rabbit & 13 & $36 \%$ \\
Dog & 8 (5 beagle) & $22 \%$ \\
Pig & 1 & $3 \%$ \\
Sheep & 1 & $3 \%$ \\
\hline
\end{tabular}

\section{Irradiation source}

The irradiation sources were Cobalt $\left(\mathrm{Co}^{60}\right)$ in 11 studies, orthovoltage in 10 studies, 4-6megavolt (MV) photons in eight studies (18 MV in two studies), and high-dose-rate (HDR) brachytherapy in four studies. One study did not mention the source of irradiation. No study used concomitant chemo-radiotherapy.

\section{Dose and fractionation}

Of the schemes mentioned, 11 studies (31\%) had a single fraction scheme with a dose ranging from 8 to 35 Gray (Gy), of which four had a brachytherapeutic source. The frequency varied from 1 to 27 fractions, and the dose given varied from 2 to $35 \mathrm{~Gy}$. In 10 studies (28\%), the fractions were given daily. Four studies had a one-week interval or more, and one study had 10 and 15 fractions given daily for a week with a one-month interval between irradiation periods.

The total dose varied from 8 to $60 \mathrm{~Gy}$. The equivalent dose, when mentioned, was between 45 and $108 \mathrm{~Gy}$.

\section{Irradiation target}

The mandible was irradiated in 34 studies, and the maxilla was included in one study. In three studies, the zygomatico-orbital complex was irradiated (table 3). 
Table 3. Irradiation target, side, and intervention.

\begin{tabular}{|c|c|c|c|c|c|c|c|}
\hline Study & Control & Total Dose & Source & Animal & Irradiation Target & Irradiation Side(s) & Intervention \\
\hline 1 & + & 24 & Co60 & Minipig & Mandible and Maxilla & Bilateral & Implantology \\
\hline 2 & - & $29,5 / 35 / 44.5$ & Ortho & Rat & Mandible & Left & Mechanical test \\
\hline 3 & - & $29,5 / 35 / 44.5$ & Ortho & Rat & Mandible & Left & Histology only \\
\hline 4 & + & 30 & Brachy & Rat & Mandible & Left & Extraction \\
\hline 5 & - & 30 & $18 \mathrm{MV}$ & Rabbit & Mandible & Bilateral & Bone transplant \\
\hline 6 & - & $32,5-45.4$ & $18 \mathrm{MV}$ & Rabbit & Mandible & Left & DO unilateral \\
\hline 7 & + & 36 & Ortho & Rat & Mandible & Left & DO unilateral \\
\hline 8 & + & 8 & Co60 & Rat & Mandible & Left & Extraction \\
\hline 9 & + & 36 & Ortho & Rat & Mandible & Left & DO unilateral \\
\hline 10 & + & 22,8 & Co60 & Dog & Mandible & Unilateral & DO unilateral \\
\hline 11 & + & 15 & Co60 & Rabbit & Mandible & Right & Implantology \\
\hline 12 & + & $15 / 25 / 35$ & Ortho & Rabbit & $\begin{array}{l}\text { ZygomaticoOrbital } \\
\text { complex }\end{array}$ & Right & Growth only \\
\hline 13 & + & 30 & Ortho & Rabbit & $\begin{array}{l}\text { ZygomaticoOrbital } \\
\text { complex }\end{array}$ & Right & Growth only \\
\hline 14 & + & $40 / 50 / 60$ & $6 \mathrm{MV}$ & Dog & Mandible & Bilateral & Implantology \\
\hline 15 & + & 43 & Co60 & Dog & Mandible & Bilateral & Implantology \\
\hline 16 & + & 22,4 & $4 \mathrm{MV}$ & Rabbit & Mandible & Bilateral & DO unilateraal \\
\hline 17 & + & 22,4 & $4 \mathrm{MV}$ & Rabbit & Mandible & Bilateral & DO unilateraal \\
\hline 18 & + & 22,4 & $4 \mathrm{MV}$ & Rabbit & Mandible & Bilateral & DO unilateraal \\
\hline 19 & + & 71,4 & Ortho & Rabbit & $\begin{array}{l}\text { ZygomaticoOrbital } \\
\text { complex }\end{array}$ & Right & Growth only \\
\hline 20 & + & 45 & $6 \mathrm{MV}$ & Sheep & Mandible & Bilateraal & DO unilateraal \\
\hline 21 & - & 27 & $6 \mathrm{MV}$ & Rabbit & Mandible & Unilateral & DO bilateral \\
\hline 22 & + & 27,5 & $6 \mathrm{MV}$ & Rabbit & Mandible & Left & DO unilateraal \\
\hline 23 & + & 20 & Brachy & Rat & Mandible & Right & Injection $\mathrm{GF}^{*}$ \\
\hline 24 & + & 20 & Brachy & Rat & Mandible & Right & Histology only \\
\hline 25 & + & 60 & $6 \mathrm{MV}$ & Rat & Mandible & Left & Histology only \\
\hline 26 & + & 42 & Ortho & Rat & Mandible & $\mathrm{nm}$ & Histology only \\
\hline 27 & + & 43 & Co60 & Dog & Mandible & Bilateral & Implantology \\
\hline 28 & - & 50 & Co60 & Dog & Mandible & $\mathrm{nm}$ & DO unilateraal \\
\hline 29 & + & 43 & Co60 & Dog & Mandible & Bilateral & Implantology \\
\hline 30 & - & 15 & Co60 & Rabbit & Mandible & Left & Implantology \\
\hline 31 & + & 32 & Ortho & Rabbit & Mandible & Left & 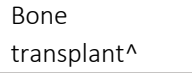 \\
\hline 32 & + & 40 & Co60 & Dog & Mandible & Uni- and Bilateral & $\begin{array}{l}\text { Bone } \\
\text { transplant\# }\end{array}$ \\
\hline 33 & + & 45 & Co60 & Rat & Mandible & Bilateral & Bone defect \\
\hline 34 & + & 54 & $\mathrm{~nm}$ & Dog & Mandible & $\mathrm{nm}$ & Bone transplant \\
\hline 35 & - & 20 & Brachy & Rat & Mandible & Left & Extraction \\
\hline 36 & + & 20 & Ortho & Rat & Mandible & Left & Growth only \\
\hline
\end{tabular}

$\mathrm{DO}=$ distraction osteogenesis, $\mathrm{GF}=$ Growth Factors being $(\mathrm{BMP}-2$ and or $\mathrm{bFGF}), \#=\mathrm{HA}$ granules ${ }^{\wedge}=$ Biphasic Calcium Phosphate Granules, $\mathrm{nm}=$ not mentioned 


\section{Intervention}

The interventions studied were distraction osteogenesis in eleven studies. Distraction osteogenesis is creating new bone from callus. The callus is formed in an artificial bone gap that is gradually being widened using an implanted 'distraction' device. In eleven studies, no intervention was performed. In other studies, the extraction of teeth, placement of dental implants, or bone transplantation into a defect combined with irradiation was studied. The interventions studied are listed in tables 3 and 4.

Table 4. Interventions performed in the included studies

\begin{tabular}{lll}
\hline Intervention & Number of studies & Percentage \\
\hline Distraction osteogenesis & 11 & $31 \%$ \\
Extractions of teeth & 3 & $8 \%$ \\
Extractions and dental implant & 7 & $19 \%$ \\
placement & $4^{*}$ & \\
Bone transplantation & 11 & $31 \%$ \\
None & & \\
\hline
\end{tabular}

* $=$ of these four studies two studies used artificial bone replacement materials

\section{Radiological evaluation}

In 11 studies, plain X-ray films and dental films were used for the radiological examinations of the irradiated bone. Four studies performed a growth analysis in nonadult growing animals on a lateral scull radiograph, five studies used micro-computed tomography (micro-CT), three studies used conventional CT images, and five studies conducted X-ray examinations of the histological sections only. Three studies evaluated the bone density using CT data, and one study performed a dual-energy X-ray absorptiometry scan. No study used magnetic resonance (MR) imaging. The timing of the radiological evaluation is listed in table 5.

\section{Radiological findings}

Studies that evaluated growth in general found growth retardation after irradiation. Studies that evaluated new bone formation following a distraction procedure reported a delay in bone formation after irradiation. Several studies produced radiographic images of the histological slides of implants and bone, thereby providing a useful evaluation of the ingrowth of dental implants. No study mentioned radiographic signs of ORN, such as erosion of the cortex, bone sequesters, or pathological fractures. 
Table 5. Irradiation target, intervention, evaluation and timing of evaluation.

\begin{tabular}{|c|c|c|c|c|c|c|}
\hline Study & Total Dose & $\begin{array}{l}\text { (Gray) } \\
\text { Source }\end{array}$ & Animal & Intervention & Evaluation & $\begin{array}{l}\text { Time after } \\
\text { irradiation }\end{array}$ \\
\hline$\overline{1}$ & 24 & Co60 & Minipig & Implantology & Radiology & 3 months \\
\hline 2 & $29,5 / 35 / 44.5$ & Ortho & Rat & Mechanical test & - & 56 days \\
\hline 3 & $29,5 / 35 / 44.5$ & Ortho & Rat & Histology only & Histology & 56 days \\
\hline 4 & 30 & Brachy & Rat & Extraction & Radiology and Histology & 28 days \\
\hline 5 & 30 & $18 \mathrm{MV}$ & Rabbit & Bone transplant & Histology & $10,20,30,90$ days \\
\hline 6 & $32,5-45.4$ & $18 \mathrm{MV}$ & Rabbit & DO unilateral & Radiology and Histology & 2,5 months \\
\hline 7 & 36 & Ortho & Rat & DO unilateral & Histology & 8 weeks \\
\hline 8 & 8 & Co60 & Rat & Extraction & Histology & 10,12 days \\
\hline 9 & 36 & Ortho & Rat & DO unilateral & Radiology & 8 weeks \\
\hline 10 & 22,8 & Co60 & Dog & DO unilateral & Radiology and Histology & 5,5 month \\
\hline 11 & 15 & Co60 & Rabbit & Implantology & Histology & $*$ \\
\hline 12 & $15 / 25 / 35$ & Ortho & Rabbit & Growth only & Radiology & 14 weeks \\
\hline 13 & 30 & Ortho & Rabbit & Growth only & Radiology & 14 weeks \\
\hline 14 & $40 / 50 / 60$ & $6 \mathrm{MV}$ & Dog & Implantology & Radiology and Histology & 12 months \\
\hline 15 & 43 & Co60 & Dog & Implantology & Radiology and Histology & 5,8 months \\
\hline 16 & 22,4 & $4 \mathrm{MV}$ & Rabbit & DO unilateral & Radiology and Histology & 13 weeks \\
\hline 17 & 22,4 & $4 \mathrm{MV}$ & Rabbit & DO unilateral & Radiology & 13 weeks \\
\hline 18 & 22,4 & $4 \mathrm{MV}$ & Rabbit & DO unilateral & Histology & 13 weeks \\
\hline 19 & 71,4 & Ortho & Rabbit & Growth only & Radiology & 21 weeks \\
\hline 20 & 45 & $6 \mathrm{MV}$ & Sheep & DO unilateral & Radiology and Histology & $\begin{array}{l}60 \text { days } \\
\text { postoperative@ }\end{array}$ \\
\hline 21 & 27 & $6 \mathrm{MV}$ & Rabbit & DO bilateral & Radiology and Histology & 5 months \\
\hline 22 & 27,5 & $6 \mathrm{MV}$ & Rabbit & DO unilateral & Radiology and Histology & 7,5 month \\
\hline 23 & 20 & Brachy & Rat & Injection GF* & Radiology and Histology & 7 weeks \\
\hline 24 & 20 & Brachy & Rat & Histology only & Histology & 100 days \\
\hline 25 & 60 & $6 \mathrm{MV}$ & Rat & Histology only & Histology & 6, 12 weeks \\
\hline 26 & 42 & Ortho & Rat & Histology only & Histology & $85,141,253$ days \\
\hline 27 & 43 & Co60 & Dog & Implantology & Radiology and Histology & 5,8 months \\
\hline 28 & 50 & Co60 & Dog & DO unilateral & Radiology and Histology & 9,5 months \\
\hline 29 & 43 & Co60 & Dog & Implantology & Radiology and Histology & 5 and 8 months \\
\hline 30 & 15 & Co60 & Rabbit & Implantology & Radiology and Histology & $\begin{array}{l}62,69,83,111 \\
\text { days }\end{array}$ \\
\hline 31 & 32 & Ortho & Rabbit & Bone transplant^^ & Radiology and Histology & 19 weeks \\
\hline 32 & 40 & Co60 & Dog & Bone transplant\# & Radiology and Histology & $0,1,2,3$ months \\
\hline 33 & 45 & Co60 & Rat & Bone defect & Histology & 6,8 weeks \\
\hline 34 & 54 & $\mathrm{~nm}$ & Dog & Bone transplant & Radiology and Histology & 4 weeks \\
\hline 35 & 20 & Brachy & Rat & Extraction & Radiology and Histology & 28 days \\
\hline 36 & 20 & Ortho & Rat & Growth only & Radiology and Histology & 30,60 days \\
\hline
\end{tabular}

$\mathrm{DO}=$ distraction osteogenesis, $\mathrm{GF}=$ Growth Factors being (BMP-2 and or bFGF), \# = HA granules ${ }^{\wedge}=$ Biphasic Calcium Phosphate Granules, $\mathrm{nm}=$ not mentioned, ${ }^{*}=7,14,30,60,90$ days after implant placement which was after 3, 6 and 12 months after irradiation, @ = 21 days after surgery irradiation started for 35 days. 


\section{Histological evaluation}

The histological staining method of the bone was most frequently hematoxylin/eosin (17 studies) and toluidine blue (six studies) staining. Some studies used multiple staining procedures. Three studies used scanning electron microscopy, and three studies used immunohistochemical analyses. Ten studies used a fluorochrome analysis of bone turnover. Often, the bone was embedded in poly(methyl methacrylate) and processed because this treatment allows for a good analysis of bone architecture and integration of dental implants. The timing of the histological evaluation is listed in table 5.

\section{Histological findings}

Several studies used semi-quantitative methods of histological evaluation, including evaluation of lacunar nucleation (empty lacunae), osteoblast/osteoclast ratio, osteocyte count, fibrosis, the presence of lamellar or woven bone, and osteoid volume. After irradiation, most studies using smaller animals reported increased fibrosis, decreased numbers of osteocytes, less vascularization, and less mature bone in distraction studies. No study reported bone necrosis.

Of the nine studies that used larger animals, including dogs and sheep, the histological findings were evaluated in detail (studies marked in gray in table 1, 3, and 5).

Effects of $50 \mathrm{~Gy}$ (equivalent dose comparable to the irradiation protocol used in humans using 2 Gy fractions)

Most studies used a 50 Gy equivalent dose. One study using Co60 irradiation and distraction osteogenesis found more immature bone in the distraction area after irradiation but found distraction osteogenesis to be feasible in the irradiated mandible 3 and 6 months after irradiation. ${ }^{24,25}$

A study using $6 \mathrm{MV}$ photon irradiation and distraction osteogenesis found significantly fewer osteoid surfaces in the regenerated bone, and there was less exuberant callus formation. However, the time of sacrifice was early, only 39 days after the beginning of irradiation treatment. ${ }^{26}$

Effects of $60 \mathrm{~Gy}$ (equivalent dose comparable to irradiation protocol used in humans using 2 Gy fractions)

Two studies that used Co60 irradiation with a reported equivalent dose of $60 \mathrm{~Gy}$ using 2 Gy fractions and implant placement reported that only three (3\%) of the 88 implants placed were mobile. There was no significant difference in the ingrowth of the implants 
in the bone between the experimental group and the control group. There were no empty lacunae, but the marrow showed strong connective tissue replacement, and arteriolar thrombosis was observed 5-8 months after irradiation. ${ }^{27,28}$

In the study using $6 \mathrm{MV}$ photon irradiation with a reported equivalent dose of $60 \mathrm{~Gy}$, all implants were mobile and lost over a period of 12 months after irradiation. ${ }^{29}$

\section{DISCUSSION}

\section{Animal choice}

The use of rat and rabbit models in most studies seems to be sufficient for an initial experimental evaluation, but their comparability to the biology of human bone remains a matter of discussion. Small animals are known to have more rapid skeletal regeneration and higher bone turnover rates. In rodents, the skeleton continuously grows and reshapes. Their growth plates remain open, and Haversian remodeling, as occurs in humans, does not occur. Haversian remodeling is a type of bone turnover with tunneling osteoclasts followed by osteoblasts forming new bone, continuously crossing the bone. Rabbits exhibit Haversian remodeling, but they have a very fatty marrow that is distinctly different from human bone marrow. ${ }^{30}$ In addition, the biology of sheep and human bone is different. Sheep bones consist mostly of a primary bone structure, unlike the largely secondary (remodeled) bones found in humans. ${ }^{31}$ Larger animals (for example, dogs and Göttingen minipigs) are more suitable than smaller animals for studies on bone biology because they are more comparable to human patients. ${ }^{31,32}$ According to Reinwald, minipig bone composition and remodeling resemble human bone biology better than those of the dog bone model. ${ }^{33}$ Additionally, the structural blood supply of the mandibles of Göttingen minipigs is comparable to that of humans. In both species, the inferior alveolar artery is the main feeding vessel of the body of the mandible and is located centrally in the bone. There are only a few anastomoses with the periosteal vessels and facial artery. This renders the bone of the mandible vulnerable to vascular damage following irradiation. ${ }^{32}$

The age of the animals should be adjusted for the study purpose. In general, head and neck oncological patients are adults, and therefore, the age of the experimental animals should be comparable.

\section{Irradiation protocol}

Of the radiation sources that were used in the different studies, orthovoltage irradiation and, to a lesser extent, $\mathrm{CO}^{60}$ irradiation, have an inferior dose distribution quality compared with 4-6 MV irradiation; however, these sources are adequate for the 
irradiation of smaller animals. In humans, the most common irradiation source is currently 4 to $6 \mathrm{MV}$ photons. ${ }^{11}$

For example, an irradiation schedule using conventional radiation therapy or IMRT involving 35 daily exposures of 2 Gy each gives a total dose of $70 \mathrm{~Gy}$, which is a common treatment schedule for patients with head and neck cancer. ${ }^{2}, 34,35$ However, in an animal experiment, it is nearly impossible to irradiate the animals according to this schedule because the animals would require daily general anesthesia for immobilization. Therefore, a compromise is usually made in reducing the number of fractions and increasing the fraction dose. The formula that is used to calculate this conversion is based on the linear quadratic model. ${ }^{35}$ For bone, the alpha/beta ratio is approximately three. ${ }^{27,36,37}$ Most studies mention the equivalent dose of the irradiation schedule compared with conventional human radiotherapy using 2 Gy fractions, but only two studies mention the calculation used. ${ }^{27,37}$

In addition, some studies used a brachytherapeutic source with a far higher maximum dose and a significant dose fall-off.

An equivalent dose of approximately 50 Gy was typically used and was applied near the mandible. This is a relatively low dose, which explains the lack of radiological evidence of irradiation damage and the relatively mild histological changes observed in these studies.

Despite the difficulties with irradiation schedules in animals, we believe that the most accurate way to simulate standard human irradiation is to apply not one but at least two fractions and to avoid acute cellular damage by using a very high irradiation dose.

Of the facial bones studied, the mandible was emphasized for two reasons. First, this bone is most often present in the irradiation field in treatments of head and neck malignancies. Second, because of its dense bone tissue and specific vascular supply, the mandible is very susceptible to ORN. However, other facial bones receive irradiation too and can develop ORN, ${ }^{38,39}$ and therefore, there is a need to study the effects of irradiation on these bones as well. In our search, we identified studies on the zygomatico-orbital complex that evaluated growth but did not evaluate histological changes. No study compared the changes in other facial bones before and after irradiation. A direct comparison would most clarify our understanding of the mechanism causing a higher incidence of ORN in the mandible compared with other facial bones.

All studies used some type of control. Seven studies performed a unilateral irradiation with a non-irradiated control side but without a separate control group. We believe that there might be an effect on the non-irradiated side, which could be partially irradiated. It is more useful to have a separate control group, as was the case in the other 28 studies. 


\section{Radiological evaluation}

In the distraction studies, the radiological evaluations generally revealed that the calcification of the distracted bone was delayed after irradiation. Studies that evaluated implant placement found more pronounced peri-implant bone loss after irradiation.

All studies show only minimal tissue changes. This is possibly because the radiological evaluation is generally performed relatively shortly after irradiation, usually after four to eight weeks. In eight studies, the specimens were evaluated six months after irradiation. Another explanation for the lack of radiological changes is that, as in humans, only 2$22 \%$ of the test animals develop ORN after irradiation.

According to Verdonck, ${ }^{40}$ bone mineral density increases on radiological evaluation following irradiation. Conversely, O'Donovan et al. mentioned a decrease in the mineral density of bone. ${ }^{41}$ One would of course expect a decrease in mineral density considering the seized bone formation. However, these contradicting findings in the absence of a clear understanding of the biological changes after irradiation warrants further investigation.

No study used MR imaging to identify the changes in bone marrow due to irradiation. MR imaging, however, is the investigation method of choice, as shown in human studies. $^{42,43}$

\section{Histological evaluation}

Histological evaluations were recorded in most studies. However, different variables and schemes were used for the semi-quantitative evaluations, making comparisons among studies impossible. The heterogeneous group of studies using different animal species, different irradiation sources, and different irradiation schedules makes direct comparisons highly problematic.

Interestingly, the large-animal experiments clearly showed differences between irradiation sources: after applying a 60 Gy equivalent dose in MV-irradiated animals, all implants were lost, ${ }^{26}$ whereas in the experiment using cobalt, ${ }^{24,}{ }^{25}$ only three of 88 implants were lost in the $\mathrm{Co}^{60}$-irradiated bone. Despite the apparently clear difference, it must be taken into account that different dose calculation and setups can explain the relevant differences. There were several histological changes that were attributed to the irradiation treatment and were more pronounced in the MV experiments. However, no empty lacunae were observed in the large animal studies, most likely because the histological evaluations were performed too early or the irradiation dose was too low. Empty lacunae arise from hypoxia because of fibrosis of the bone marrow and thrombosis with endarteritis, which is a late effect of irradiation.

Describing histological results is difficult, and the use of semi-quantitative methods helps to standardize the results. Despite the helpful scoring system of Heiple (modified by Zhang), inter- and intra-observer variability remains a problem when comparing the 
results of different studies. ${ }^{44}$ The parameters used were empty lacunae or osteocyte counts and, to a lesser extent, marrow fibrosis. These parameters should be used in a standardized manner in future studies to facilitate comparability, as shown by Fenner et al.. ${ }^{37}$ Despite the large number of studies evaluated, it is not possible to directly compare these studies in detail because they were descriptive in nature.

The questions posed earlier remain mostly unanswered due to the different animal models, applied radiation dose, irradiation source, observation period, and evaluation of results.

However, some clinical implications of the results from these studies can be found: implants placed in irradiated bone with an equivalent dose of 60 Gray have a poor success rate compared to 50 Gray, implants placed before irradiation have a higher success rate compared to implants placed after irradiation, distraction osteogenesis seems feasible after irradiation, Amifostine clearly preserves cell function after irradiation, and Hyperbaric Oxygen therapy is reported to have some beneficial effects.

Based on this review, we designed an experiment that started in 2012 to develop an animal model for osteoradionecrosis. The results of this experiment will be published in the near future. Since January 2012, several new studies have been published on the irradiation of facial bones. For now, these studies do not fundamentally change our view on this subject, but they will be discussed further in our future publications.

\section{Recommendations}

Based on these studies we can recommend the following: when designing a new animal experiment on the irradiation of facial bones, one should take several factors into account. Large animals, such as dogs and minipigs, should be used for translational research when comparing the results to those of human patients. The ideal irradiation source is $6 \mathrm{MV}$ photons. There should be at least two fractions. Because irradiation effects on bone are late effects, at least three months should pass, preferably longer, before performing radiological and histological evaluations.

In histological evaluations, quantitative and semi-quantitative measurements should include osteocyte counts and assessments of marrow fibrosis.

In radiological evaluations, the plain X-ray and $\mathrm{CT}$ examinations should be performed after enough time has passed, at least three months, to be able to observe radiological changes of the bone caused by irradiation. It would furthermore be best to evaluate radiological changes of the bone marrow following irradiation with MR imaging, as this is considered to be the gold standard.

Future research should aim to find radioprotectants minimizing radiation damage to the bone and prevent ORN. Experimental investigation of ORN treatment with pentoxiphilin and tocopherol protocol by Delenian can further help us to understand the pathophysiology and refine the treatment of ORN. 
CHAPTER 2

\section{Acknowledgements}

We kindly thank professor Lambin for his valuable comments on our manuscript. 


\section{REFERENCES}

1. Klug C, Wutzl A, Kermer C, Voracek M, Kornek G, Selzer E, Glaser C, Poeschl PW, Millesi W and Ewers R, Preoperative radiochemotherapy and radical resection for stages II-IV oral and oropharyngeal cancer: outcome of 222 patients. Int J Oral Maxillofac Surg, 2005. 34(2): p. 143-8.

2. Paximadis PA, Christensen ME, Dyson G, Kamdar DP, Sukari A, Lin HS, Yoo GH and Kim HE, Up-front neck dissection followed by concurrent chemoradiation in patients with regionally advanced head and neck cancer. Head Neck, 2012.

3. Ramaekers BL, Pijls-Johannesma M, Joore MA, van den Ende P, Langendijk JA, Lambin P, Kessels AG and Grutters JP, Systematic review and meta-analysis of radiotherapy in various head and neck cancers: comparing photons, carbon-ions and protons. Cancer Treat Rev, 2011. 37(3): p. 185-201.

4. Egelmeer AG, Velazquez ER, de Jong JM, Oberije C, Geussens Y, Nuyts S, Kremer B, Rietveld D, Leemans CR, de Jong MC, Rasch C, Hoebers F, Homer J, Slevin N, West C, and Lambin P, Development and validation of a nomogram for prediction of survival and local control in laryngeal carcinoma patients treated with radiotherapy alone: a cohort study based on 994 patients. Radiother Oncol, 2011. 100(1): p. 108-15.

5. Studer G, Studer SP, Zwahlen RA, Huguenin P, Gratz KW, Lutolf UM and Glanzmann C, Osteoradionecrosis of the mandible: minimized risk profile following intensity-modulated radiation therapy (IMRT). Strahlenther Onkol, 2006. 182(5): p. 283-8.

6. Ramaekers BL, Joore MA, Grutters JP, van den Ende P, Jong J, Houben R, Lambin P, Christianen M, Beetz I, Pijls-Johannesma $M$, and Langendijk JA, The impact of late treatment-toxicity on generic health-related quality of life in head and neck cancer patients after radiotherapy. Oral Oncol, 2011. 47(8): p. 768-74.

7. Christianen ME, Langendijk JA, Westerlaan HE, van de Water TA and Bijl HP, Delineation of organs at risk involved in swallowing for radiotherapy treatment planning. Radiother Oncol, 2011. 101(3): p. 394-402.

8. Overgaard J, Hypoxic modification of radiotherapy in squamous cell carcinoma of the head and neck--a systematic review and meta-analysis. Radiother Oncol, 2011. 100(1): p. 22-32.

9. Ben-David MA, Diamante M, Radawski JD, Vineberg KA, Stroup C, Murdoch-Kinch CA, Zwetchkenbaum SR and Eisbruch A, Lack of osteoradionecrosis of the mandible after intensity-modulated radiotherapy for head and neck cancer: likely contributions of both dental care and improved dose distributions. Int J Radiat Oncol Biol Phys, 2007. 68(2): p. 396-402.

10. Lee J and Moon C, Current status of experimental therapeutics for head and neck cancer. Exp Biol Med (Maywood), 2011. 236(4): p. 375-89.

11. Thariat J, Bolle S, Demizu Y, Marcy PY, Hu Y, Santini J, Bourhis J and Pommier P, New techniques in radiation therapy for head and neck cancer: IMRT, CyberKnife, protons, and carbon ions, Improved effectiveness and safety? Impact on survival? Anticancer Drugs, 2011. 22: p. 596-606.

12. Mul VE, de Jong JM, Murrer LH, van den Ende PL, Houben RM, Lacko M, Lambin P and Baumert BG, Lhermitte sign and myelopathy after irradiation of the cervical spinal cord in radiotherapy treatment of head and neck cancer. Strahlenther Onkol, 2012. 188(1): p. 71-6.

13. Boomsma MJ, Bijl HP and Langendijk JA, Radiation-induced hypothyroidism in head and neck cancer patients: a systematic review. Radiother Oncol, 2011. 99(1): p. 1-5.

14. Teng MS and Futran ND, Osteoradionecrosis of the mandible. Curr Opin Otolaryngol Head Neck Surg, 2005. 13(4): p. 217-21.

15. Grabenbauer GG, Rodel C, Brunner T, Schulze-Mosgau S, Strnad V, Muller RG, Iro H and Sauer R, Interstitial brachytherapy with Ir-192 low-dose-rate in the treatment of primary and recurrent cancer of the oral cavity and oropharynx. Review of 318 patients treated between 1985 and 1997. Strahlenther Onkol, 2001. 177(7): p. 338-44.

16. Lyons A and Ghazali N, Osteoradionecrosis of the jaws: current understanding of its pathophysiology and treatment. Br J Oral Maxillofac Surg, 2008. 46(8): p. 653-60.

17. Marx RE and Johnson RP, Studies in the radiobiology of osteoradionecrosis and their clinical significance. Oral Surg Oral Med Oral Pathol, 1987. 64(4): p. 379-90. 
18. Jereczek-Fossa BA and Orecchia R, Radiotherapy-induced mandibular bone complications. Cancer Treat Rev, 2002. 28(1): p. 65-74.

19. Jacobson AS, Buchbinder D, Hu K and Urken ML, Paradigm shifts in the management of osteoradionecrosis of the mandible. Oral Oncol, 2010. 46(11): p. 795-801.

20. Notani K, Yamazaki Y, Kitada H, Sakakibara N, Fukuda H, Omori K and Nakamura M, Management of mandibular osteoradionecrosis corresponding to the severity of osteoradionecrosis and the method of radiotherapy. Head Neck, 2003. 25(3): p. 181-6.

21. Bras J, de Jonge HK and van Merkesteyn JP, Osteoradionecrosis of the mandible: pathogenesis. Am J Otolaryngol, 1990. 11(4): p. 244-50.

22. Marx RE and Tursun R, Suppurative osteomyelitis, bisphosphonate induced osteonecrosis, osteoradionecrosis: a blinded histopathologic comparison and its implications for the mechanism of each disease. Int J Oral Maxillofac Surg, 2012. 41(3): p. 283-9.

23. Zhuang $\mathrm{Q}$, Zhang $\mathrm{Z}, \mathrm{Fu} \mathrm{H}, \mathrm{He} \mathrm{J}$ and $\mathrm{He} \mathrm{Y}$, Does radiation-induced fibrosis have an important role in pathophysiology of the osteoradionecrosis of jaw? Med Hypotheses, 2011. 77(1): p. 63-5.

24. Liu Y, Liu G, Liu $Y$ and Lin $X$, Character of distracted bone in irradiated canine mandibles and electrophysiological changes in the inferior alveolar nerve. Br J Oral Maxillofac Surg, 2010. 48(2): p. 11520.

25. Gantous A, Phillips JH, Catton P and Holmberg D, Distraction osteogenesis in the irradiated canine mandible. Plast Reconstr Surg, 1994. 93(1): p. 164-8.

26. Girod A, Roger $T$, Breton $P$ and Bouletreau $P$, Experimental study of mineralization in mandibular bone distraction with irradiation during the consolidation phase. J Craniomaxillofac Surg, 2005. 33(6): p. 386-94.

27. Brogniez V, D'Hoore W, Gregoire V, Munting E and Reychler H, Implants placed in an irradiated dog mandible: a morphometric analysis. Int J Oral Maxillofac Implants, 2000. 15(4): p. 511-8.

28. Brasseur M, Brogniez V, Gregoire V, Reychler H, Lengele B, D'Hoore W and Nyssen-Behets C, Effects of irradiation on bone remodelling around mandibular implants: an experimental study in dogs. Int J Oral Maxillofac Surg, 2006. 35(9): p. 850-5.

29. Asikainen P, Klemetti E, Kotilainen R, Vuillemin T, Sutter F, Voipio HM and Kullaa A, Osseointegration of dental implants in bone irradiated with 40, 50 or 60 gy doses. An experimental study with beagle dogs. Clin Oral Implants Res, 1998. 9(1): p. 20-5.

30. Muschler GF, Raut VP, Patterson TE, Wenke JC and Hollinger JO, The design and use of animal models for translational research in bone tissue engineering and regenerative medicine. Tissue Eng Part B Rev, 2010. 16(1): p. 123-45.

31. Pearce Al, Richards RG, Milz S, Schneider E and Pearce SG, Animal models for implant biomaterial research in bone: a review. Eur Cell Mater, 2007. 13: p. 1-10.

32. Saka B, Wree A, Anders L and Gundlach KK, Experimental and comparative study of the blood supply to the mandibular cortex in Gottingen minipigs and in man. J Craniomaxillofac Surg, 2002. 30(4): p. 219-25.

33. Reinwald S and Burr D, Review of nonprimate, large animal models for osteoporosis research. J Bone Miner Res, 2008. 23(9): p. 1353-68.

34. Rusthoven KE, Raben D, Ballonoff A, Kane M, Song Jl and Chen C, Effect of radiation techniques in treatment of oropharynx cancer. Laryngoscope, 2008. 118(4): p. 635-9.

35. Fowler JF, 21 years of biologically effective dose. Br J Radiol, 2010. 83(991): p. 554-68.

36. Fowler JF, Tome WA, Fenwick JD and Mehta MP, A challenge to traditional radiation oncology. Int J Radiat Oncol Biol Phys, 2004. 60(4): p. 1241-56.

37. Fenner M, Park J, Schulz N, Amann K, Grabenbauer GG, Fahrig A, Karg J, Wiltfang J, Neukam FW and Nkenke $E$, Validation of histologic changes induced by external irradiation in mandibular bone. An experimental animal model. J Craniomaxillofac Surg, 2010. 38(1): p. 47-53.

38. Lim AA, Karakla DW and Watkins DV, Osteoradionecrosis of the cervical vertebrae and occipital bone: a case report and brief review of the literature. Am J Otolaryngol, 1999. 20(6): p. 408-11.

39. Yoo JS, Rosenthal DI, Mitchell K and Ginsberg LE, Osteoradionecrosis of the hyoid bone: imaging findings. AJNR Am J Neuroradiol, 2010. 31(4): p. 761-6. 
40. Verdonck HW, Meijer GJ, Nieman FH, Stoll C, Riediger D and de Baat C, Quantitative computed tomography bone mineral density measurements in irradiated and non-irradiated minipig alveolar bone: an experimental study. Clin Oral Implants Res, 2008. 19(5): p. 465-8.

41. O'Donovan DA, Yeung I, Zeman V, Neligan PC, Pang CY and Forrest CR, Radiation-induced craniofacial bone growth inhibition: development of an animal model. J Craniofac Surg, 2001. 12(6): p. 533-43.

42. Store G, Smith HJ and Larheim TA, Dynamic MR imaging of mandibular osteoradionecrosis. Acta Radiol, 2000. 41(1): p. 31-7.

43. Rabin BM, Meyer JR, Berlin JW, Marymount MH, Palka PS and Russell EJ, Radiation-induced changes in the central nervous system and head and neck. Radiographics, 1996. 16(5): p. 1055-72.

44. Zhang WB, Zheng LW, Chua D and Cheung LK, Bone regeneration after radiotherapy in an animal model. J Oral Maxillofac Surg, 2010. 68(11): p. 2802-9. 


\section{APPENDIX 1.}

\section{Studies included}

1. Verdonck HW, Meijer GJ, Laurin T, Nieman FH, Stoll C, Riediger D, Stoelinga PJ and de Baat C, Assessment of vascularity in irradiated and nonirradiated maxillary and mandibular minipig alveolar bone using laser doppler flowmetry. Int J Oral Maxillofac Implants, 2007. 22(5): p. 774-8.

2. Tchanque-Fossuo CN, Monson LA, Farberg AS, Donneys A, Deshpande SS, Razdolsky ER, Halonen NR, Goldstein SA and Buchman SR, Dose-response effect of human equivalent radiation in the murine mandible: Part II. A biomechanical assessment. Plast Reconstr Surg. 128(5): p. 480e-487e.

3. Tchanque-Fossuo CN, Monson LA, Farberg AS, Donneys A, Zehtabzadeh AJ, Razdolsky ER and Buchman $\mathrm{SR}$, Dose-response effect of human equivalent radiation in the murine mandible: part I. A histomorphometric assessment. Plast Reconstr Surg. 128(1): p. 114-21.

4. Cohen M, Nishimura I, Tamplen M, Hokugo A, Beumer J, Steinberg ML, Suh JD, Abemayor E and Nabili V, Animal model of radiogenic bone damage to study mandibular osteoradionecrosis. Am J Otolaryngol. 32(4): p. 291-300.

5. Yachouh J, Breton P, Roux JP and Goudot P, Osteogenic capacity of vascularised periosteum: an experimental study on mandibular irradiated bone in rabbits. J Plast Reconstr Aesthet Surg. 63(12): p. 2160-7.

6. Zhang WB, Zheng LW, Chua D and Cheung LK, Bone regeneration after radiotherapy in an animal model. J Oral Maxillofac Surg, 2010. 68(11): p. 2802-9.

7. Inyang AF, Schwarz DA, Jamali AM and Buchman SR, Quantitative histomorphometric assessment of regenerate cellularity and bone quality in mandibular distraction osteogenesis after radiation therapy. J Craniofac Surg. 21(5): p. 1438-42.

8. Hosokawa Y, Sakakura Y, Tanaka L, Okumura K, Yajima T and Kaneko M, Effects of local and whole body irradiation on appearance of osteoclasts during wound healing of tooth extraction sockets in rats. J Radiat Res (Tokyo), 2007. 48(4): p. 273-80.

9. Fregene A, Jing XL, Monson LA and Buchman SR, Alteration in volumetric bone mineralization density gradation patterns in mandibular distraction osteogenesis following radiation therapy. Plast Reconstr Surg, 2009. 124(4): p. 1237-44.

10. Liu Y, Liu G, Liu $Y$ and Lin $X$, Character of distracted bone in irradiated canine mandibles and electrophysiological changes in the inferior alveolar nerve. Br J Oral Maxillofac Surg, 2010. 48(2): p. 11520.

11. Matsui $Y$, Ohno K, Michi K and Tachikawa T, Histomorphometric examination of healing around hydroxylapatite implants in 60Co-irradiated bone. J Oral Maxillofac Surg, 1994. 52(2): p. 167-72; discussion 172-3.

12. O'Donovan DA, Yeung I, Zeman V, Neligan PC, Pang CY and Forrest CR, Radiation-induced craniofacial bone growth inhibition: development of an animal model. J Craniofac Surg, 2001. 12(6): p. 533-43.

13. Forrest CR, O'Donovan DA, Yeung I, Zeman V, La Scala G, Neligan PC and Pang CY, Efficacy of radioprotection in the prevention of radiation-induced craniofacial bone growth inhibition. Plast Reconstr Surg, 2002. 109(4): p. 1311-23; discussion 1324.

14. Asikainen $P$, Klemetti $E$, Kotilainen $R$, Vuillemin $T$, Sutter F, Voipio HM and Kullaa A, Osseointegration of dental implants in bone irradiated with 40, 50 or 60 gy doses. An experimental study with beagle dogs. Clin Oral Implants Res, 1998. 9(1): p. 20-5.

15. Brogniez V, Nyssen-Behets C, Gregoire V, Reychler H and Lengele B, Implant osseointegration in the irradiated mandible. A comparative study in dogs with a microradiographic and histologic assessment. Clin Oral Implants Res, 2002. 13(3): p. 234-42.

16. Muhonen A, Muhonen J, Lindholm TC, Minn H, Klossner J, Kulmala J and Happonen RP, Osteodistraction of a previously irradiated mandible with or without adjunctive hyperbaric oxygenation: an experimental study in rabbits. Int J Oral Maxillofac Surg, 2002. 31(5): p. 519-24. 
17. Muhonen A, Muhonen J, Minn H, Kulmala J, Klossner J, Teras M, Knuuti J and Happonen RP, The effects of irradiation and hyperbaric oxygen on bone formation during rabbit mandibular distraction. Arch Oral Biol, 2002. 47(10): p. 701-7.

18. Muhonen A, Peltomaki T, Knuuti J, Raitakari O and Happonen RP, Osteoblastic activity of the rabbit temporomandibular joint during distraction osteogenesis assessed by [18F]fluoride positron emission tomography. Eur J Oral Sci, 2002. 110(2): p. 144-8.

19. La Scala GC, O'Donovan DA, Yeung I, Darko J, Addison PD, Neligan PC, Pang CY and Forrest CR, Radiationinduced craniofacial bone growth inhibition: efficacy of cytoprotection following a fractionated dose regimen. Plast Reconstr Surg, 2005. 115(7): p. 1973-85.

20. Girod A, Roger T, Breton P and Bouletreau P, Experimental study of mineralization in mandibular bone distraction with irradiation during the consolidation phase. J Craniomaxillofac Surg, 2005. 33(6): p. 38694.

21. Shao Z, Liu B, Liu Y, Liu W, Liu R, Peng Q and Liu L, Distraction osteogenesis in the irradiated rabbit mandible. J Plast Reconstr Aesthet Surg, 2006. 59(2): p. 181-7.

22. Clark CL, Strider J, Hall C, Ferguson HW, Armstrong KL, Runner RR and Baur DA, Distraction osteogenesis in irradiated rabbit mandibles with adjunctive hyperbaric oxygen therapy. J Oral Maxillofac Surg, 2006. 64(4): p. 589-93.

23. Springer IN, Niehoff $P$, Acil $Y$, Marget $M$, Lange A, Warnke $P H$, Pielenz $H$, Roldan JC and Wiltfang J, BMP-2 and bFGF in an irradiated bone model. J Craniomaxillofac Surg, 2008. 36(4): p. 210-7.

24. Niehoff P, Springer IN, Acil Y, Lange A, Marget M, Roldan JC, Koppe K, Warnke PH, Kimmig B and Wiltfang J, HDR brachytherapy irradiation of the jaw - as a new experimental model of radiogenic bone damage. J Craniomaxillofac Surg, 2008. 36(4): p. 203-9.

25. Fenner M, Park J, Schulz N, Amann K, Grabenbauer GG, Fahrig A, Karg J, Wiltfang J, Neukam FW and Nkenke $E$, Validation of histologic changes induced by external irradiation in mandibular bone. An experimental animal model. J Craniomaxillofac Surg, 2010. 38(1): p. 47-53.

26. Williamson RA, An experimental study of the use of hyperbaric oxygen to reduce the side effects of radiation treatment for malignant disease. Int J Oral Maxillofac Surg, 2007. 36(6): p. 533-40.

27. Brasseur M, Brogniez V, Gregoire V, Reychler H, Lengele B, D'Hoore W and Nyssen-Behets C, Effects of irradiation on bone remodelling around mandibular implants: an experimental study in dogs. Int J Oral Maxillofac Surg, 2006. 35(9): p. 850-5.

28. Gantous A, Phillips JH, Catton P and Holmberg D, Distraction osteogenesis in the irradiated canine mandible. Plast Reconstr Surg, 1994. 93(1): p. 164-8.

29. Brogniez V, D'Hoore W, Gregoire V, Munting E and Reychler H, Implants placed in an irradiated dog mandible: a morphometric analysis. Int J Oral Maxillofac Implants, 2000. 15(4): p. 511-8.

30. Schon R, Ohno K, Kudo M and Michi K, Peri-implant tissue reaction in bone irradiated the fifth day after implantation in rabbits: histologic and histomorphometric measurements. Int J Oral Maxillofac Implants, 1996. 11(2): p. 228-38.

31. Jegoux F, Aguado E, Cognet R, Malard O, Moreau F, Daculsi G and Goyenvalle E, Alveolar ridge augmentation in irradiated rabbit mandibles. J Biomed Mater Res A. 93(4): p. 1519-26.

32. Pinholt EM and Kwon $\mathrm{PH}$, The effect of therapeutic radiation on canine alveolar ridges augmented with hydroxylapatite. J Oral Maxillofac Surg, 1992. 50(3): p. 250-4.

33. Lorente CA, Song BZ and Donoff RB, Healing of bony defects in the irradiated and unirradiated rat mandible. J Oral Maxillofac Surg, 1992. 50(12): p. 1305-9.

34. Klotch DW, Ganey T, Greenburg H and Slater-Haase A, Effects of radiation therapy on reconstruction of mandibular defects with a titanium reconstruction plate. Otolaryngol Head Neck Surg, 1996. 114(4): p. 620-7.

35. Tamplen M, Trapp K, Nishimura I, Armin B, Steinberg M, Beumer J, Abemayor E and Nabili V, Standardized analysis of mandibular osteoradionecrosis in a rat model. Otolaryngol Head Neck Surg. 145(3): p. 404-10.

36. Ubios AM, Piloni MJ and Cabrini RL, Mandibular growth and tooth eruption after localized x-radiation. J Oral Maxillofac Surg, 1992. 50(2): p. 153-6. 



\section{CHAPTER 3}

\section{Assessment of local blood flow with laser doppler flowmetry in irradiated mandibular and frontal bone, an experiment in Göttingen minipigs.}

L.J. Poort, M.M.F. Bloebaum, R.A. Böckmann, R. Houben, M.E.P.G. Granzier, F.J.P. Hoebers, P.A.W.H. Kessler

Accepted in the Journal of Cranio-Maxillo-Facial Surgery 


\section{ABSTRACT}

Purpose The aim of this study was to investigate local blood flow changes in the mandibular bone compared to the os frontale after irradiation in various doses.

Materials and Methods This study used an animal experiment with 16 female Göttingen minipigs. Three groups of four animals were irradiated with equivalent doses of 25, 50 or 70 Gray on the mandible and os frontale and four animals served as control. Three months after irradiation laser doppler flowmetry (LDF) was used to record local blood flow on the left mandible and in the irradiated area on the os frontale. At 6 months measurements were repeated. Descriptive and univariate analyses were conducted and p-values lower than 0,05 were considered statistically significant.

Results Local blood flow measurements in the mandible, were significantly higher compared to the os frontale. In the os frontale and mandible there was no significant change in the measurements with increasing irradiation dose.

Conclusion We found a non-significant decrease in LDF values with an increase in radiation dose in the mandible and non-significant changes in the os frontale at 3 and 6 months. We consider this to represent the process of ongoing fibrosis affecting the local blood flow in the mandible. 


\section{INTRODUCTION}

In general, for head and neck malignancies, treatment regimens often include radiotherapy because of its beneficial effects on recurrence and survival. ${ }^{1-3}$ However, irradiation effects on the surrounding healthy tissue continue to cause complications of varying severity. ${ }^{4-7}$ One complication is osteoradionecrosis (ORN). The diagnosis is generally based on clinical presentation. ORN is defined as "irradiated bone, which becomes devitalized, is exposed through the overlying skin or mucosa, and does not heal within a period of 3 months without tumor recurrence" ${ }^{8}$ It can arise at any point after irradiation therapy. ${ }^{9}$

ORN can affect all bony structures in the head and neck region but is most commonly seen in the mandible, at a frequency of $2 \%-22 \% .{ }^{10}$

Pathophysiology of ORN is, according to Delanian et al, a state in which radiationinduced fibrosis accounts for the damage observed in normal tissues, including bone, after radiotherapy. The destruction of endothelial cells coupled with vascular thrombosis leads to the necrosis of microvessels, local ischemia, and tissue loss. This leads to the key event in this type of damage - the activation and deregulation of fibroblastic activity. ${ }^{8}$

The treatment of ORN ranges from conservative management, with or without surgical debridement, to broad resection in severe cases, a major surgical intervention. ${ }^{11-13}$

In earlier experiments, laser doppler flowmetry (LDF) was used for recording microvascular blood flow in cancellous mandibular bone of young pigs. ${ }^{14}$ LDF has also been shown to be useful in the assessment of bone vitality in osteomyelitis and in other applications. $^{15-17}$

LDF technique uses a laser diode with an operating wavelength of 780 to $820 \mathrm{~nm}$, which is emitted into human tissues. Photons are scattered, and light hitting moving blood cells undergoes a shift in wavelength (Doppler shift) while the wavelength of light hitting static structures remains unchanged. A returning fiber in the probe collects the scattered light. The magnitude of the signal and the frequency changes is directly related to the relative number and velocity of blood cells in a given volume. ${ }^{18}$

To understand the effects of irradiation on blood flow in bone, we used an animal experiment already partially described in a previous publication. ${ }^{19}$ The irradiation and measurements were conducted on the os frontale and mandible. The os frontale and mandible have distinct anatomies and embryological origins. ${ }^{20}$ The os frontale is composed almost entirely of dense cortical bone. Its vasculature is like a web with many anastomoses. The mandible has a dense outer cortex with loose trabecular bone in the marrow space. The vascularization of the mandible relies mainly on the inferior alveolar artery and lacks sufficient collaterals. This makes the mandible more vulnerable to vascular damage after irradiation. ${ }^{8,21}$ 
The aim of this study was to investigate changes in local blood flow of mandibular bone compared to os frontale after irradiation in various doses.

\section{MATERIALS AND METHODS}

The experiment was performed in accordance with the European Community Guidelines for the Protection of (laboratory) Animals as described in our earlier publication. ${ }^{19}$ Permission was obtained from the local Animal Ethical Committee (DEC 2011-127).

\section{Study design}

The study design was an animal experiment using 16 healthy, adult 18-month-old female Göttingen minipigs.

\section{Study variables}

4 animals were randomly assigned to each of the 4 research groups receiving an equivalent radiation dose of 25, 50 or 70 Gray (Gy) and one control goup. The primary outcome variable was median LDF value. The primary predictor variables were radiation dose in different groups and measurement site. Other variables such as age and sex were constant for all animals.

\section{Data collection methods}

In preparing for the irradiation, for the three irradiated groups and the control group a planning CT-scan was made under general anesthesia. A thermoplastic immobilization mask was employed for optimal fixation of the animal's head. A circular light metal wire was placed to mark the irradiation field on the os frontale. A Siemens Sensation Open ${ }^{\text {TM }}$ CT-scan was used for radiological imaging (Siemens, Erlangen, Germany).

The animals were irradiated under general anesthesia. The animals were positioned in individualized thermoplastics molds for reproducible and steady positioning.

The irradiation was delivered using a 6 megavolt linear accelerator with two opposing lateral beams (Siemens, Erlangen, Germany). The entire body of the mandible was irradiated in two fractions with equivalent doses of 25, 50 or $70 \mathrm{~Gy}$ (two fractions of 6.5, 9.7, and $11.8 \mathrm{~Gy}$, respectively). The same treatment was administered to the central portion of the os frontale with an external electron beam. An alpha/beta ratio for late responding tissues was used to calculate the equivalent dose. We assumed the alpha/beta ratio to be 3 . The second fraction was given 48 hours after the first fraction. In each group every animal received the same equivalent irradiation dose for the 
mandible and for the frontal bone. None of the above was done in the control (0 Gy) group.

13 weeks after irradiation, surgery was performed under general anesthesia. The surgical procedure on the mandible involved extraction of all premolars and molars on the left side. Using a pilot drill of the implant system (BioComp Industries BV, Vught, the Netherlands), 4 initial holes were drilled in the residual alveolar ridge. To assess bone vascularity, laser doppler flowmetry (Periflux System Perimed, Järfälla, Sweden) recordings were carried out at a depth of approximately $6 \mathrm{~mm}$. After signal stabilization, a 20 second recording was carried out in every initial hole. Four recordings were made with the probe directed perpendicularly to the mesial, buccal, lingual and distal wall successively (figure 1 and 2). 4 submerged dental implants (diameter 4,0 mm and length $12 \mathrm{~mm}$ ) were placed in each pig. The measurements on the mandible took place on the side of the surgery in order to study both the effects of irradiation alone (in os frontale) and in combination with surgical intervention (in the mandible).

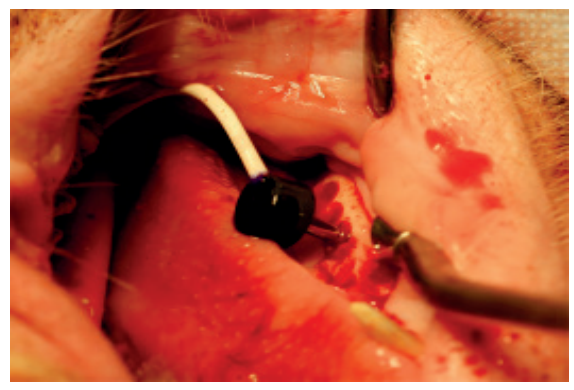

Figure 1. Laser Doppler flowmetry measurements mandible

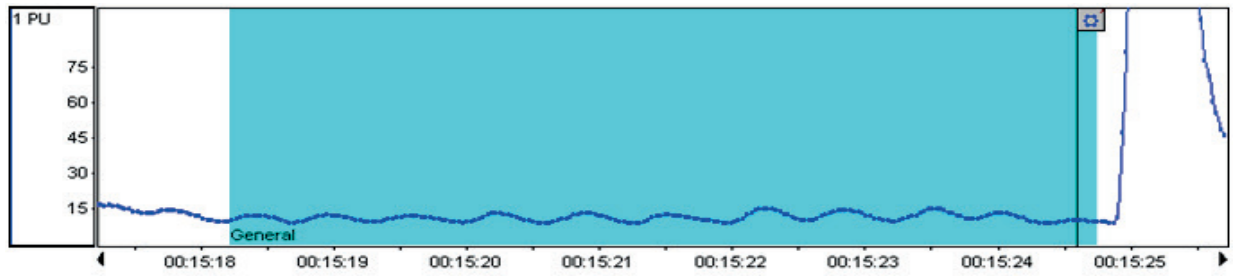

Figure 2. Laser doppler flowmetry graph of the mandible. (y-axis: $P U=$ perfusion unit, $x$-axis time in hour:minute:seconds)

A small dose of methylene blue stain was injected into the periosteum of the frontal bone, marking the irradiated area. Then, an incision was made over the os frontale exposing the underlying bone. 4 holes were drilled in the irradiated bone using the pilot drill and drill guide. To assess bone vascularity, LDF recordings were made at a depth of approximately $3 \mathrm{~mm}$ in the irradiated area (figure 3 and 4). The recordings were also 
conducted with the probe perpendicular to the wall of the initial hole in the above mentioned 4 directions. A median of all four values for each hole was later used for the statistical analysis. In that same area, 4 submerged dental implants (diameter 4,0 $\mathrm{mm}$ and length $4,0 \mathrm{~mm}$ ) were placed in each pig. After rinsing the wound, it was closed in layers using resorbable sutures. The animals received intramuscular antibiotics (amoxicillin, AST beheer BV, Oudewater, the Netherlands) and intramuscular pain medication (buprenorphine, Reckitt Benckiser Healthcare, Hull, United Kingdom) for three days postoperatively.

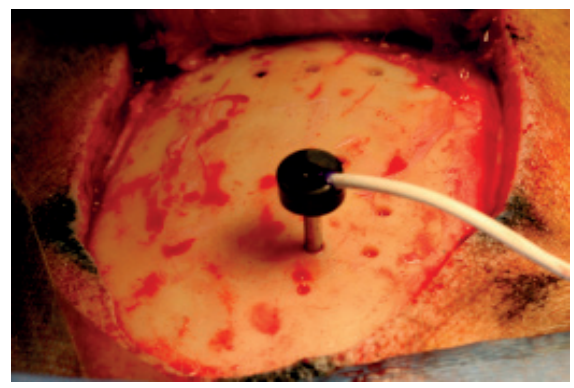

Figure 3. Doppler flowmetry measurements on the os frontale.

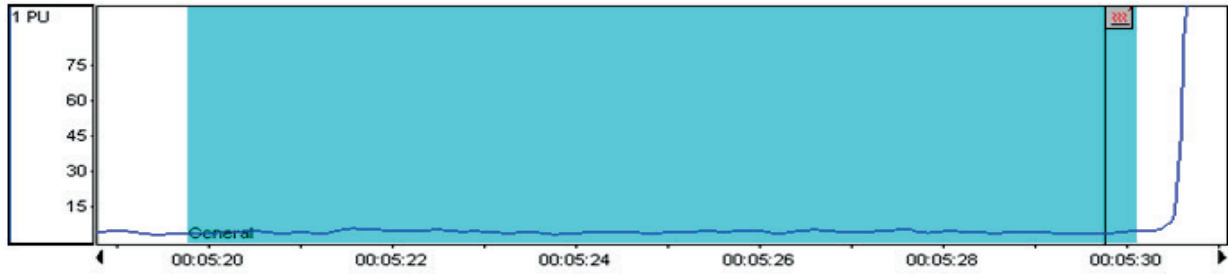

Figure 4. Laser doppler flowmetry graph of the os frontale. (y-axis: PU = perfusion unit, $x$-axis time in hour:minute:seconds)

At 26 weeks after irradiation a small incision was made in the irradiated area on the os frontale and on the mandible, distal to the canine tooth on each side. After one bur hole was made with the pilot drill in each location, LDF recordings were made.

\section{Data analyses}

The data were incrementally entered during the study period into an electronic sheet (Excel $^{\mathrm{TM}}$, Microsoft Office 2010, Redmond, WA, USA), and then processed using SPSS $20^{\mathrm{Tm}}$ (Statistical Package for the Social Sciences, SPSS Inc., Chicago, IL, USA).

Statistics for description of continuous variables and median values are given with the standard deviation and minimum and maximum. For statistical analysis, median LDF 
values for each group and each location were used. The Independent-Samples KruskalWallis test, and Independent-Samples Mann-Whitney $U$ test were used for comparison of the median LDF values. P-values $\leq 0.05$ were considered significant.

\section{RESULTS}

The median LDF values of the irradiated minipig os frontales and mandibles are listed in table 1 and corresponding box plots are shown in figures 5a, 5b, 6a and 6b. These LDF values represent the magnitude and frequency changes of the signal; these attributes are directly related to the number and velocity of blood cells in a recorded volume in the irradiated bone.

Table 1. Median Laser doppler flowmetry (LDF) values at 3 and 6 months after irradiation of both os frontale and mandible for the groups 0, 25, 50 and 70 Gray equivalent dose (Mann-Whitney $U$ test).

\begin{tabular}{|c|c|c|c|c|c|c|c|c|}
\hline Os frontale & 0 Gray & & 25 Gray & & 50 Gray & & 70 Gray & \\
\hline months & 3 & 6 & 3 & 6 & 3 & 6 & 3 & 6 \\
\hline Median & 6,7 & 18,5 & 5,2 & 6,0 & 6,6 & 13,2 & 7,7 & 8,2 \\
\hline$p$-value & $0,029 *$ & & 0,886 & & 0,057 & & 0,686 & \\
\hline Min & 2,8 & 13,4 & 5,0 & 1,7 & 4,2 & 7,6 & 3,3 & 7,2 \\
\hline Max & 10,4 & 25,8 & 8,5 & 28,8 & 8,8 & 59,7 & 9,1 & 11,2 \\
\hline Mandible & O Gray & & 25 Gray & & 50 Gray & & 70 Gray & \\
\hline months & 3 & 6 & 3 & 6 & 3 & 6 & 3 & 6 \\
\hline Median & 33,3 & 15,2 & 24,3 & 10,7 & 19,4 & 14,6 & 19,9 & 9,5 \\
\hline$p$-value & $0,029 *$ & & 0,057 & & 0,343 & & $0,029 *$ & \\
\hline Min & 30,1 & 12,7 & 15,0 & 9,1 & 16,4 & 6,4 & 16,3 & 8,5 \\
\hline Max & 36,7 & 20,0 & 39,5 & 18,9 & 45,2 & 21,2 & 27,6 & 10,6 \\
\hline
\end{tabular}

By comparing all median LDF values at 3 months between the mandible and the os frontale, significantly higher median LDF values for the mandible were noted.

By comparing all median LDF values at 6 months between the mandible and the os frontale, no significant differences were noted.

By comparing the median LDF values in the os frontale of all irradiated groups, only a non-significant decrease by increasing the radiation dose at 3 months $(p=0,96)$ and at 6 months $(p=0,19)$ were noted.

By comparing the median LDF values in the mandible of all irradiated groups, again only an non-significant decrease by increasing the radiation dose at 3 months $(p=0,23)$ and at 6 months $(p=0,22)$ were noted.

By comparing the median LDF values at 3 and 6 months for the os frontale and separately for the mandible, only non-significant changes were noted. The only 


\section{CHAPTER 3}

exception was the significant decrease in the median LDF values in the os frontale for the 70 Gy group.

By comparing the median LDF values at 3 months and at 6 months in the control (0 Gy) group, a statistically significant increase for the os frontale and a significant decrease for the mandible were noted.
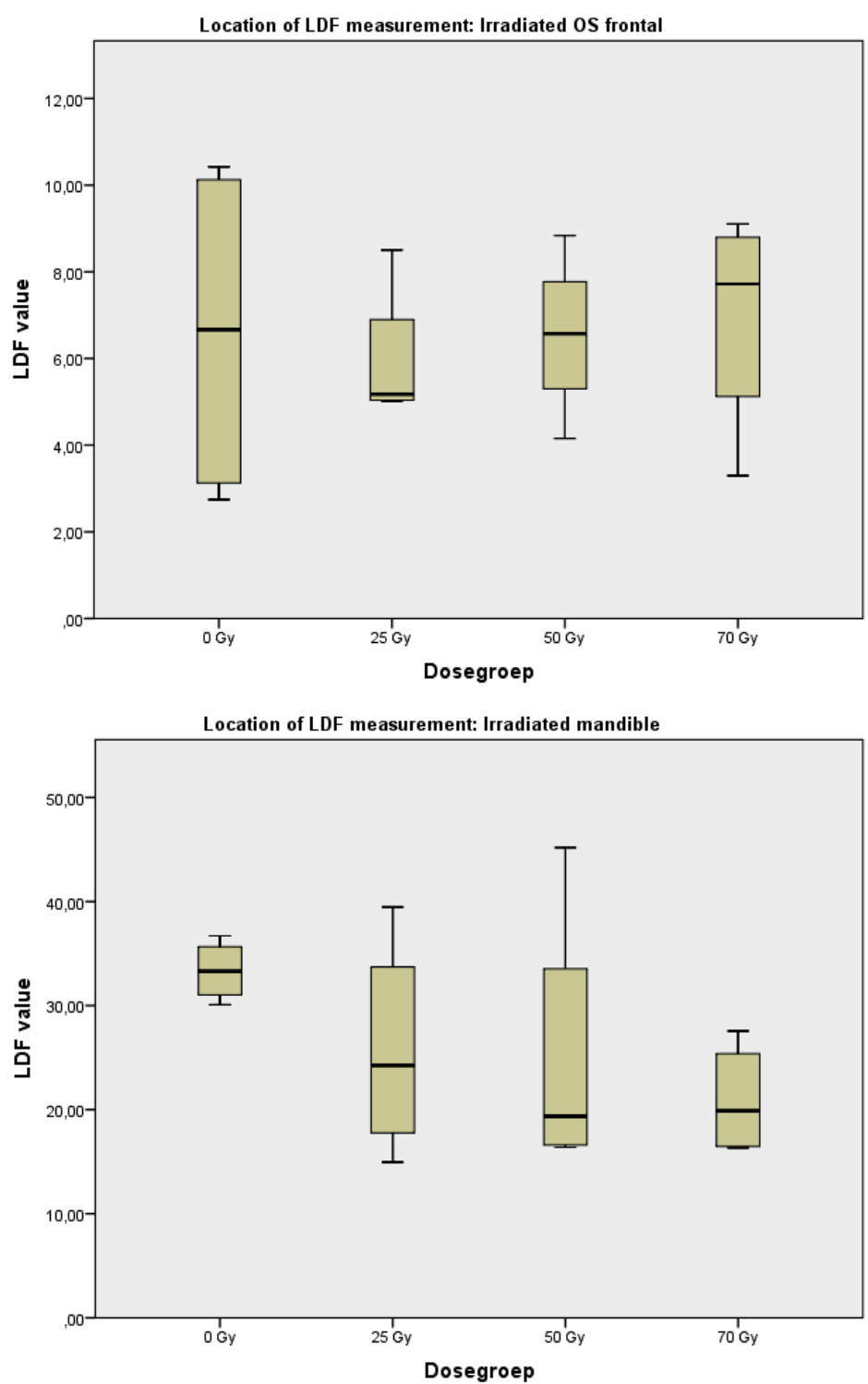

Figure $5 \mathrm{a}$ and $\mathrm{b}$. Box plots of the median laser doppler flowmetry (LDF) values at 3 months after irradiation of the os frontale and mandible. (Kruskal-Wallis test os frontale $p=0,962$, mandible $p=0,231$ ) 

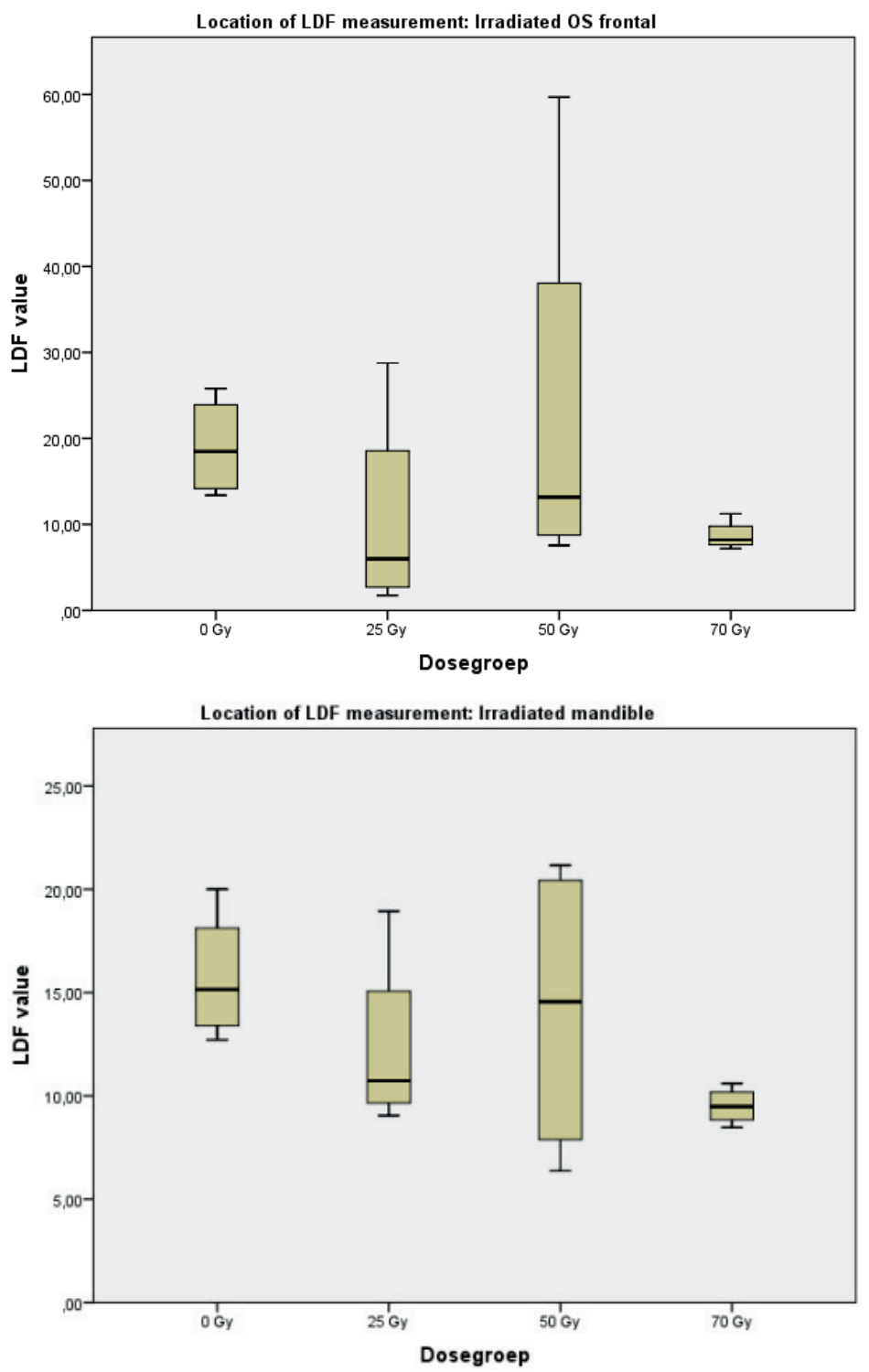

Figure $6 a$ and $b$. Box plots of the median laser doppler flowmetry (LDF) values at 6 months after irradiation of the os frontale and mandible. (Kruskal-Wallis test os frontale $p=0,188$, mandible $p=0,220$ )

\section{DISCUSSION}

The purpose of the present study was to investigate changes in local blood flow of mandibular bone compared to os frontale after irradiation in various doses. The 
hypothesis of this study was that local blood flow of both mandible and os frontale diminishes increasingly after irradiation with to increasing radiation dose. Due to the different characteristics of os frontale and mandible we expected a greater decrease in blood flow in the mandible compared to the os frontale.

In our study we found that for median LDF values at 3 and at 6 months post irradiation there was no significant difference between dose groups in either location. Except for the significant decrease in the median LDF values in the os frontale in the 70 Gy group, there was a non-significant decrease in median LDF values. That is clearly visible in the boxplots of the mandible after both 3 and 6 months. We interpret this to be the pathophysiological process of gradual fibrosis, although it is not statistically proven in our study, making this a clinically relevant model for studying the development of osteoradionecrosis. In the boxplots of median LDF values of the os frontale there was no visible decrease at 3 and 6 months post irradiation.

When comparing the values between 3 and 6 months a problem arises. In the 0 Gy group of the os frontale, there was a significant increase of the median LDF value between 3 and 6 months, whereas in the 0 Gray group for the mandible, there was a significant decrease of the median LDF values during the same period. Several factors can explain the significant changes in the control group. One factor was that minor changes in the measurement location, between 3 months and 6 months cause change in local blood flow measurements of the mandible. Because LDF measurements cause local tissue destruction, a limited number of measurements were taken in different locations to allow for future histological examination. The measurements on the mandible at 3 months were made on the left side only. At 6 months only one location of the os frontale and the mandible were measured. Clinically, the quality of mandibular bone surrounding the canine was more dense, corresponding with lower vascularity measurements. The measurements on the os frontale at 6 months were made centrally in the irradiated area. The midline of the frontal bone is slightly thicker due to the presence of the sagittal suture which is composed of lower density bone, corresponding with higher vascularity measurements.

As noted by Verdonck et al, despite the reproducibility of the LDF measurements, there is variation with measurement direction, site and the individual animal. ${ }^{22}$ Because of this variation, it is important to measure LDF as a mean of measurements in 4 directions and compare similar localizations. Additionally, this variation means that minor changes of the measurement locations at 6 months cause significant changes in the control group, making comparisons between the LDF values at 3 and 6 months questionable. Another reason may be the small amount of animals in each group. If we had tested a larger number of animals, and if the measurements had been taken without further surgical interventions, we believe that the differences between groups would have been statistically significant. Finally there might have be minimal differences in the equivalent 
dose for the different locations and the different dose groups, possibly causing differences between the measurements.

Interesting observations were the relatively high LDF values in the 50 Gy group in the mandible and, to a lesser extent, in the os frontale. This might represent the relatively strong compensatory increase in vascularity for repair of damaged bone after irradiation in this group. The narrowing of the range of LDF measurements at $70 \mathrm{~Gy}$ of both os frontale and mandible were interesting as well. We interpret this to be a pathophysiological process of gradual fibrosis, making this a clinically relevant animal model for studying the development of osteoradionecrosis.

A recent study by $X u$ et al. showed an initial increase of LDF value in blood flow in the pig mandible only a few days after irradiation. This was followed by a decrease of LDF value at 2 weeks post-operatively and a gradual recovery at 4 weeks after irradiation. These values remained significantly lower than normal. For this study, they used irradiation with one fraction of $25 \mathrm{~Gy}$ from a $6 \mathrm{MV}$ external beam source (equivalent dose of $130 \mathrm{~Gy}$, calculated with linear quadratic model using an alpha/beta ratio of 3). ${ }^{23}$ The study performed by Verdonck et al. using minipigs, measured LDF in mandibular bone 3 months after irradiation and found a significant decrease in LDF with cobalt ${ }^{60}$ irradiation (3 weekly fractions of $8 \mathrm{~Gy}$, equivalent dose of $53 \mathrm{~Gy}$, calculated with linear quadratic model using an alpha/beta ratio of 3). ${ }^{22}$ The small changes in LDF recorded in our study might be as a result of the fact that the recordings were made directly after extraction of the left molars and premolars, causing vascular disturbances. Consequently, a wider range of LDF values needs a larger number of animals to detect significant changes between the irradiation dose groups. Another explanation for the smaller changes is the fractionation and equivalent doses. Xu et al. used a single fraction irradiation protocol while we used a 2 fraction protocol. In our study only 3 animals had clinical osteoradionecrosis, whereas in the study of $\mathrm{Xu}$ et al, all animals had clinical osteoradionecrosis. ${ }^{23}$

In this study, the change in local blood flow in the bone after irradiation was too small to detect in these relatively small groups. Due to variations between localizations and individual methodologies, careful data interpretation is necessary for using this technique in humans. However, we believe, that measuring local blood flow using invasive LDF not a viable option, because of the risk on inducing ORN as a result of the surgical intervention.

Future research on this animal model should be conducted to measure the effect on local blood flow after irradiation with medication that prevents fibrosis such as pentoxifillin, tocoferol and clodronate. Compared to the few large animal studies in LDF measurement that we found in the literature, we must state, that the irradiation protocol used in our experiment seems to be a realistic model for the understanding of the development of ORN in human beings. 


\section{CONCLUSION}

This study presents an animal model to simulate tissue reactions induced by various doses of radiation in the mandible and os frontale. We found a non-significant decrease in LDF values with increase in radiation dose most clearly in the mandible. We consider this to represent the process of ongoing fibrosis affecting local blood flow after irradiation, although our initial hypothesis could not be statistically approved. We conclude also, that the change in the measurement location at 3 and 6 months, the tooth extraction on the mandible at 3 months and the two-fraction irradiation protocol might also have affected the measurements and led to unexpected statistical findings. The small test groups might also have contributed to the compromised statistical analysis. New experiments with larger test groups and more precise experiment protocols need to be conducted to confirm our conclusions.

\section{ACKNOWLEDGEMENTS}

We kindly thank BioComp Industries BV, Vught, the Netherlands, for partially funding this animal experiment. 


\section{REFERENCES}

1. Klug C, Wutzl A, Kermer C, Voracek M, Kornek G, Selzer E, Glaser C, Poeschl PW, Millesi W and Ewers R, Preoperative radiochemotherapy and radical resection for stages II-IV oral and oropharyngeal cancer: outcome of 222 patients. Int J Oral Maxillofac Surg, 2005. 34(2): p. 143-8.

2. Paximadis PA, Christensen ME, Dyson G, Kamdar DP, Sukari A, Lin HS, Yoo GH and Kim HE, Up-front neck dissection followed by concurrent chemoradiation in patients with regionally advanced head and neck cancer. Head Neck, 2012.

3. Ramaekers BL, Pijls-Johannesma M, Joore MA, van den Ende P, Langendijk JA, Lambin P, Kessels AG and Grutters JP, Systematic review and meta-analysis of radiotherapy in various head and neck cancers: comparing photons, carbon-ions and protons. Cancer Treat Rev, 2011. 37(3): p. 185-201.

4. Lee J and Moon C, Current status of experimental therapeutics for head and neck cancer. Exp Biol Med (Maywood), 2011. 236(4): p. 375-89.

5. Thariat J, Bolle S, Demizu Y, Marcy PY, Hu Y, Santini J, Bourhis J and Pommier P, New techniques in radiation therapy for head and neck cancer: IMRT, Cyberknife, protons, and carbon ions, Improved effectiveness and safety? Impact on survival? Anticancer Drugs, 2011. 22: p. 596-606.

6. Mul VE, de Jong JM, Murrer LH, van den Ende PL, Houben RM, Lacko M, Lambin P and Baumert BG, Lhermitte sign and myelopathy after irradiation of the cervical spinal cord in radiotherapy treatment of head and neck cancer. Strahlenther Onkol, 2012. 188(1): p. 71-6.

7. Boomsma MJ, Bijl HP, Christianen ME, Beetz I, Chouvalova O, Steenbakkers RJ, van der Laan BF, Wolffenbuttel BH, Oosting SF, Schilstra C, and Langendijk JA, A prospective cohort study on radiationinduced hypothyroidism: development of an NTCP model. Int J Radiat Oncol Biol Phys, 2012. 84(3): p. e351-6.

8. Lyons A and Ghazali N, Osteoradionecrosis of the jaws: current understanding of its pathophysiology and treatment. Br J Oral Maxillofac Surg, 2008. 46(8): p. 653-60.

9. Marx RE and Johnson RP, Studies in the radiobiology of osteoradionecrosis and their clinical significance. Oral Surg Oral Med Oral Pathol, 1987. 64(4): p. 379-90.

10. Teng MS and Futran ND, Osteoradionecrosis of the mandible. Curr Opin Otolaryngol Head Neck Surg, 2005. 13(4): p. 217-21.

11. Jereczek-Fossa BA and Orecchia R, Radiotherapy-induced mandibular bone complications. Cancer Treat Rev, 2002. 28(1): p. 65-74.

12. Notani K, Yamazaki Y, Kitada H, Sakakibara N, Fukuda H, Omori K and Nakamura M, Management of mandibular osteoradionecrosis corresponding to the severity of osteoradionecrosis and the method of radiotherapy. Head Neck, 2003. 25(3): p. 181-6.

13. Jacobson AS, Buchbinder $\mathrm{D}, \mathrm{Hu} \mathrm{K}$ and Urken $\mathrm{ML}$, Paradigm shifts in the management of osteoradionecrosis of the mandible. Oral Oncol, 2010. 46(11): p. 795-801.

14. Hellem S, Jacobsson LS, Nilsson GE and Lewis DH, Measurement of microvascular blood flow in cancellous bone using laser Doppler flowmetry and 133Xe-clearance. Int J Oral Surg, 1983. 12(3): p. 16577.

15. Swiontkowski MF, Hagan K and Shack RB, Adjunctive use of laser Doppler flowmetry for debridement of osteomyelitis. J Orthop Trauma, 1989. 3(1): p. 1-5.

16. Duwelius PJ and Schmidt AH, Assessment of bone viability in patients with osteomyelitis: preliminary clinical experience with laser Doppler flowmetry. J Orthop Trauma, 1992. 6(3): p. 327-32.

17. Teh J, Applications of Doppler imaging in the musculoskeletal system. Curr Probl Diagn Radiol, 2006. 35(1): p. 22-34.

18. Michelson G, Schmauss B, Langhans MJ, Harazny J and Groh MJ, Principle, validity, and reliability of scanning laser Doppler flowmetry. J Glaucoma, 1996. 5(2): p. 99-105.

19. Poort $\amalg$ BG, Böckmann RA, Hoebers FJ, Houben R, Postma AA, Kessler PAWH Is there a change in bone mineral density changes of the mandible in Göttingen minipigs after irradiation in correlation with 


\section{CHAPTER 3}

radiation dose and implant surgery? Journal of Oral and Maxillofacial Surgery, 2014. 72(11): p. 21492156.

20. Carlson B, Embryology \& Developmental Biology. Mosby., 1999: p. 166-170.

21. Zhuang $\mathrm{Q}$, Zhang $\mathrm{Z}, \mathrm{Fu} \mathrm{H}$, He J and He $\mathrm{Y}$, Does radiation-induced fibrosis have an important role in pathophysiology of the osteoradionecrosis of jaw? Med Hypotheses, 2011. 77(1): p. 63-5.

22. Verdonck HW, Meijer GJ, Laurin T, Nieman FH, Stoll C, Riediger D, Stoelinga PJ and de Baat C, Assessment of vascularity in irradiated and nonirradiated maxillary and mandibular minipig alveolar bone using laser doppler flowmetry. Int J Oral Maxillofac Implants, 2007. 22(5): p. 774-8.

23. Xu J, Zheng Z, Fang D, Gao R, Liu Y, Fan ZP, Zhang CM and Wang SL, Early-stage pathogenic sequence of jaw osteoradionecrosis in vivo. J Dent Res, 2012. 91(7): p. 702-8. 


\section{CHAPTER 4}

Does a Change in Bone Mineral Density occur in the Mandible of Göttingen Minipigs after Irradiation in Correlation With Radiation Dose and Implant Surgery?

L.J. Poort, G.K.P. Bittermann, R.A. Böckmann, F.J.P. Hoebers, R. Houben, A.A. Postma, P.A.W.H. Kessler

Published in the Journal of Oral and Maxillofacial Surgery 2014;72(11):2149-56. 


\section{ABSTRACT}

Purpose: To date, studies have not agreed on the effects of irradiation on bone mineral density. The aim of our study was to investigate the changes in mandibular bone mineral density after irradiation at various doses with and without surgery.

Materials and Methods: We implemented a descriptive animal experiment. The sample included 16 female Göttingen Minipigs, randomly assigned to 4 groups and irradiated with equivalent doses of $0,25,50$, and 70 Gy to the mandibular region. At 3 months after irradiation, the mandibular left premolars and molars were removed, and dental implants were placed. Computed tomography scans were taken before and 6 months after irradiation. The measured bone density was related to a bone phantom to calculate the bone mineral density quotient (BMDQ). The outcome variable was the $B M D Q$. Other study variables were the radiation dose and surgery. Descriptive and univariate analyses were computed, and significance was set at $\mathrm{P} \leq 0.05$.

Results: In the left hemi-mandible, compared with the control group, a significant decrease in BMDQ was observed: 0.01 at $0 \mathrm{~Gy},-0.01$ at $25 \mathrm{~Gy},-0.06$ at $50 \mathrm{~Gy}$, and -0.11 at $70 \mathrm{~Gy}(\mathrm{P}=0.023)$. The right hemi-mandible compared with the control group also showed a significant decrease in BMDQ: -0.02 at $0 \mathrm{~Gy},-0.08$ at $25 \mathrm{~Gy},-0.09$ at $50 \mathrm{~Gy}$, and -0.11 at $70 \mathrm{~Gy}(\mathrm{P}=0.007)$.

Conclusions: The present study used a large animal model to simulate the tissue reactions induced by various radiation doses in the mandible. We found a significant decrease in the BMDQ after irradiation, but no significant correlation could be found between the irradiation dose and a decrease in the BMDQ. 


\section{INTRODUCTION}

Radiotherapy (RT) is one of the treatment options for malignancies of the head and neck region. However, RT results in side effects on healthy tissue. The most serious complication in the head and neck region is the development of osteoradionecrosis (ORN). ${ }^{1}$ The definition most widely used for ORN has been "irradiated bone, which becomes devitalized and is exposed through the overlying skin or mucosa and does not heal within a period of three months, without tumor recurrence" ${ }^{2}$ Patient factors that will increase the risk of the development of ORN include age, gender, health, nutritional status, and the use of tobacco and alcohol. ${ }^{3-5}$ The treatment of ORN is challenging, because it often involves major surgery and causes high morbidity.

RT-induced ORN of the mandible was described as early as $1930 .^{4}$ However, when modern photon therapy with megavolt energy levels is applied in RT, the high-energy doses are absorbed differently by the bone compared with the kilovolt energy doses used previously. Before modern technology, the incidence of ORN in the mandible was reported as high as $22 \%$. Current data have reported an incidence of mandibular ORN after RT of approximately 5\%, most likely because of more precise and refined irradiation protocols. ${ }^{1,3}$ Various hypotheses have been proposed about the pathophysiology of ORN. According to Marx and Marx and Johnson, ORN is caused by a combination of vascular and cellular changes in the mandibular bone. ${ }^{6,7}$ Histologic evaluation has shown acellularity and avascularity of the bone marrow and a nonviable periosteum. This condition develops in irradiated areas, leading to tissue hypoxia. Hypoxic conditions cause direct damage to the bone cells, the osteoclasts and osteoblasts. ${ }^{6,7}$ The cell damage is initiated by 2 different pathways: the direct effect of radiation on the bone and tissue cells and the effect of free radicals damaging the healthy cells. Tissue repair due to iatrogenic or non-iatrogenic trauma will no longer be possible. Tissue breakdown, however, can also be seen spontaneously, without any trauma. ${ }^{2}$ According to Delanian et al. and Delanian and Lefaix, 3 phases can be discerned in the tissue reaction to irradiation. ${ }^{5,8}$ This reaction has been termed "radiation-induced fibroatrophy" (RIF). The initial prefibrotic phase includes damage to the endothelial cells. The loss of the endothelial cells results in exposure of the connective tissue cells to normally foreign stimuli, which could trigger fibroblastic activation. After the initial phase, RIF induces tissue growth composed of active fibroblasts (myofibroblasts), senescent fibroblasts (fibrocytes), and extracellular matrix. The combined damage to the endothelial cells and the connective tissue cells, amplified by the action of cytokines, leads to a permanent state of RIF. Finally, remodeling of the extracellular matrix progressively increases the tissue density, leaving friable tissue with poor vascularization and cellularized fibroatrophy. These areas are prone to developing ORN. ${ }^{5,8}$

How these pathophysiologic processes affect bone quality and density is not clear. To date, studies have not agreed on the changes in bone mineral density (BMD) after RT. ${ }^{9-12}$ It is still unclear whether BMD measurements will aid in assessing the severity of bone damage. 
The purpose of the present study was twofold. First, we intended to introduce a large animal model to investigate the BMD changes in the mandibles of Göttingen

Minipigs irradiated with various radiation doses. Second, we wished to investigate a possible correlation between BMD changes and the radiation dose, with and without surgical intervention. Our hypothesis was, according to the pathophysiology and findings of Verdonck et al, that we would expect a small increase in the BMD quotient (BMDQ) after irradiation. ${ }^{9}$ The specific aim of our study was to develop a tool to measure the quality of the irradiated bone. We tried to more closely emulate the clinical human patient in the animal model by using a split mouth model to be able to compare both sides of the mandible, performing surgery on the left side only. The surgical intervention consisted of extractions and implant placement to simulate clinical reality. The bone density was measured adjacent to the implants to prove the clinical concept of implant-based prostheses in the animal model. Quantitative computed tomography (CT) was used to evaluate the BMD changes. ${ }^{9,13}$

\section{MATERIALS AND METHODS}

The experiment was performed in accordance with the European Community Guidelines for the Protection of (Laboratory) Animals. The local animal ethical committee approved the study.

\section{Study design}

The study design was an animal experiment. For the present study, 16 healthy adult 18month-old female Göttingen Minipigs were used. There were no additional inclusion or exclusion criteria.

\section{Study variables}

The predictor variable in the present study was the irradiation dose group. Four pigs were randomly assigned to each of the 4 research groups stratified by the radiation dose received $(0,25,50$, and 70 Gy, respectively). The primary outcome variable was the BMDQ. The other study variables were radiation dose and surgery (yes or no). The primary predictor variables were radiation dose and surgery. Other variables, such as age and gender, were the same for all the pigs. No other variables were related to the outcome. 


\section{Data collection methods}

For RT planning purposes, a preparatory planning CT scan was taken with the pigs under general anesthesia with endotracheal intubation. A thermoplastic immobilization mask was used for optimal fixation of the minipig's head. A Siemens Sensation Open CT scanner was used for radiologic documentation (Siemens, Erlangen, Germany). Axial slices of $1 \mathrm{~mm}$ were taken. The CT scans were performed with a calibrated bone phantom placed in the mouth of the minipig to calculate the relative BMDQ and absolute bone density.9,13 At 26 weeks after irradiation, another non-enhanced CT scan was taken. The procedure was the same as that before irradiation.

The minipigs underwent irradiation under general anesthesia with endotracheal intubation. They were positioned in an individualized thermoplastics mold for reproducible and steady positioning. Irradiation was delivered with a 6-megavolt linear accelerator with 2 opposing lateral beams (Siemens). The entire body of the mandible was irradiated in 2 fractions with an equivalent dose of $0,25,50$, or 70 Gy (2 fractions of $0,6.5,9.7$, and $11.8 \mathrm{~Gy}$ ). To calculate the equivalent dose, an a/b ratio for lateresponding tissues was used. We assumed that the $a / b$ ratio was 3 . The second fraction was given 48 hours after the first fraction. At 13 weeks after irradiation, surgery was performed with the minipigs under general anesthesia with endotracheal intubation and mechanical ventilation. Established protocols for animal care were used to guarantee maximum safety.

To simulate a translational clinical situation, the surgical procedure on the mandible involved extraction of all premolars and molars on the left side. Next, 4 submerged BioComp dental implants (diameter $4.0 \mathrm{~mm}$ and length $12 \mathrm{~mm}$ ) were placed in each pig (BioComp Industries BV, Vught, The Netherlands). The pigs received intramuscular antibiotics (amoxicillin) and intramuscular pain medication (buprenorphine), which were continued for 3 days postoperatively.

Using SimPlant 15 (Materialize, Leuven, Belgium), the data from the $2 \mathrm{CT}$ scans were analyzed. To measure the BMD, we used a region of interest (ROI) of 5 to $10 \mathrm{~mm} 2$. The $\mathrm{ROI}$ tool for measuring bone density was placed on 5 different predetermined locations of the mandibular cortex on both sides of the mandible (Fig 1). Cortical bone was deliberately chosen to eliminate measurement errors caused by dental or implant material. Because the cortical bone contains less collagenous tissue than bone marrow, its measurements of BMD have been considered to be more accurate. Two of us (L.P., G.B.) performed the measurements independently from one another. The calibrated bone phantom had a predetermined density given in $\mathrm{g} / \mathrm{mL}$. The BMDQ was calculated by dividing the measurement by the average BMD of the phantom (Fig 2). The BMDQ in the present study was used to quantitatively compare the changes in the BMD. The BMD values represented the degree of mineralization of bone. The BDM is known to be relatively low in immature newly formed bone compared with the mature bone matrix. 

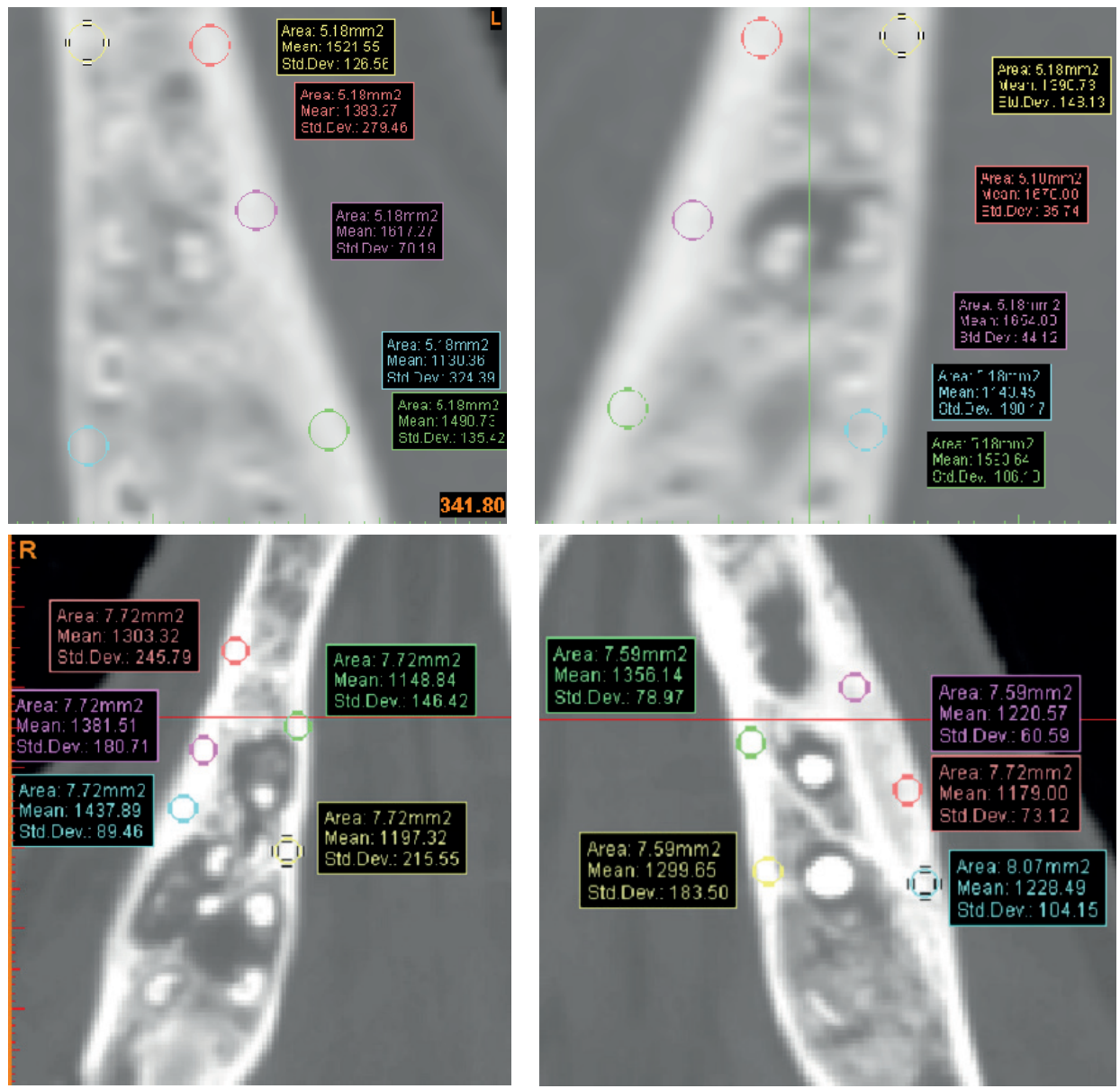

Figure 1. Location of the circular region of interest. A, Preirradiation right mandible, B, left mandible, C, postirradiation right mandible, and $D$, implanted left mandible (SimPlant 15, Materialise, Leuven, Belgium). Std. Dev = standard deviation.

\section{Statistical analysis}

The data were incrementally entered during the study period into an electronic sheet (Excel, Microsoft Office 2010, Microsoft, Redmond, WA) and then processed using IBM SPSS Statistics, version 20 (IBM, Armonk, NY). First, to test the reproducibility of the measurements of the 2 independent investigators, we calculated the agreement between the measurements using the Bland-Altman procedure. ${ }^{14}$ The statistical significance of the changes in BMD in relation to the radiation dose was determined using the Kruskal-Wallis test. The statistical significance of the changes in bone density per location was determined using the Wilcoxon signed rank test. The Kruskal-Wallis 
test for independent samples was chosen to evaluate the relationship between the radiation dose and the BMDQ in different locations. We also compared the BMDQ of all the locations before and after RT using the Wilcoxon signed rank test. Because of the small sample and corresponding small power, we used only univariate analyses and nonparametric tests, because the data could not be assumed to be normally distributed, and significance was set at $\mathrm{P} \leq .05$.

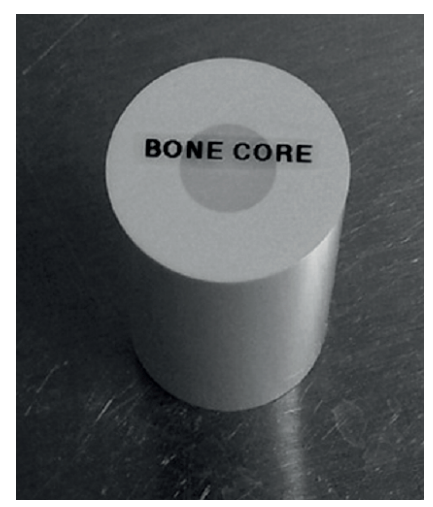

Figure 2. Photograph of the bone phantom used in this experiment

\section{RESULTS}

The BMDQ values of the non-irradiated and irradiated mandibles of the minipigs are listed in Table 1. These values represent the difference in the BMD of the mandible compared with a calibrated bone phantom. The absolute values should not be extrapolated to those of other BMD data from human patients. In our experiment, a significant decrease was found in the BMDQ after RT in the left (surgically altered) mandible, with a significant decrease in the right (non-surgically altered) mandibular side. Between the left and right side of the mandible, no significant differences $(P=.023$ for the left mandible, $P=.007$ for the right mandible, and $P=.564$ for the difference between the left and right mandibles) were found. In the non-irradiated mandible, no significant change was seen in the BMDQ between the first and second CT scan. Although no significant correlation was found between the radiation dose and the decrease in $\mathrm{BMDQ}$ on both sides (left side, $\mathrm{P}=.134$; right side, $\mathrm{P}=.599$ ), a progressive decrease in the BMDQ was visible on both sides. In Figure 3, the Bland-Altman graph is shown and demonstrated the agreement of the measurements of the 2 independent investigators. Nearly all the measurements lay within the calculated standard deviation (SD-2 ranges; ie, the so-called limits of agreement). 
Table 1. Bone mineral density quotient before and after irradiation

\begin{tabular}{|c|c|c|c|c|c|c|}
\hline & \multicolumn{2}{|c|}{ BMDQ before irradiation } & \multicolumn{4}{|c|}{ BMDQ after irradiation } \\
\hline & $\begin{array}{l}\text { Mandible left } \\
\text { (intervention) }\end{array}$ & Mandible right & $\begin{array}{l}\text { Mandible left } \delta \\
\text { (intervention) }\end{array}$ & (\%) & Mandible rig & $\begin{array}{l}\delta \\
(\%)\end{array}$ \\
\hline \multicolumn{7}{|l|}{ No irradiation } \\
\hline $\begin{array}{l}\text { Median } \\
\text { (min-max) }\end{array}$ & $\begin{array}{l}1,72 \\
(1,63-1,74)\end{array}$ & $\begin{array}{l}1,71 \\
(1,64-1,79)\end{array}$ & $\begin{array}{l}1,73 \\
(1,61-1,77)\end{array}$ & $\begin{array}{l}0,01 \\
(0,6 \%)\end{array}$ & $\begin{array}{l}1,69 \\
(1,55-1,74)\end{array}$ & $\begin{array}{l}-0,02 \\
(-1,2 \%)\end{array}$ \\
\hline \multicolumn{7}{|l|}{$25 \mathrm{~Gy}$} \\
\hline $\begin{array}{l}\text { Median } \\
\text { (min-max) }\end{array}$ & $\begin{array}{l}1,64 \\
(1,44-1,77)\end{array}$ & $\begin{array}{l}1,71 \\
(1,63-1,78)\end{array}$ & $\begin{array}{l}1,63 \\
(1,52-1,64)\end{array}$ & $\begin{array}{l}-0,01 \\
(-0,6 \%)\end{array}$ & $\begin{array}{l}1,63 \\
(1,52-1,74)\end{array}$ & $\begin{array}{l}-0,08 \\
(-4,7 \%)\end{array}$ \\
\hline \multicolumn{7}{|l|}{50 Gy } \\
\hline $\begin{array}{l}\text { Median } \\
\text { (min-max) }\end{array}$ & $\begin{array}{l}1,63 \\
(1,62-1,78)\end{array}$ & $\begin{array}{l}1,73 \\
(1,45-1,79)\end{array}$ & $\begin{array}{l}1,57 \\
(1,48-1,63)\end{array}$ & $\begin{array}{l}-0,06 \\
(-3.7 \%)\end{array}$ & $\begin{array}{l}1,64 \\
(1,34-1,72)\end{array}$ & $\begin{array}{l}-0,09 \\
(-5,2 \%)\end{array}$ \\
\hline \multicolumn{7}{|l|}{$70 \mathrm{~Gy}$} \\
\hline $\begin{array}{l}\text { Median } \\
\text { (min-max) }\end{array}$ & $\begin{array}{l}1,67 \\
(1,57-1,72)\end{array}$ & $\begin{array}{l}1,73 \\
(1,63-1,79)\end{array}$ & $\begin{array}{l}1,56 \\
(1,54-1,66)\end{array}$ & $\begin{array}{l}-0,11 \\
(-6,6 \%)\end{array}$ & $\begin{array}{l}1,62 \\
(1,40-1,64)\end{array}$ & $\begin{array}{l}-0,11 \\
(-6,4 \%)\end{array}$ \\
\hline
\end{tabular}

$P=.134$ and $P=.599$ for comparisons among the irradiation groups after irradiation for the left mandible and right mandible, respectively; $P=.023$ and $P=.007$ for comparisons between before and after irradiation for the left mandible and right mandible, respectively; differences were statistically significant at $\mathrm{P}<.05$.

Abbreviations: BMDQ, bone mineral density quotient (calculated using the average of a known bone phantom (measured Hounsfield unit value of the area divided by the known value of the phantom); d, difference between the BMDQ value before and after irradiation.

Thus, an adequate agreement was present between the measurements of the 2 investigators. Two pigs developed clinical ORN: 1 in the 50-Gy and 1 in the 70-Gy group. Both pigs developed bone sequestration on the surgically altered mandibular side. Despite this clinical finding, the BMDQ of these individual pigs did not differ significantly from the remaining pigs in their groups. 


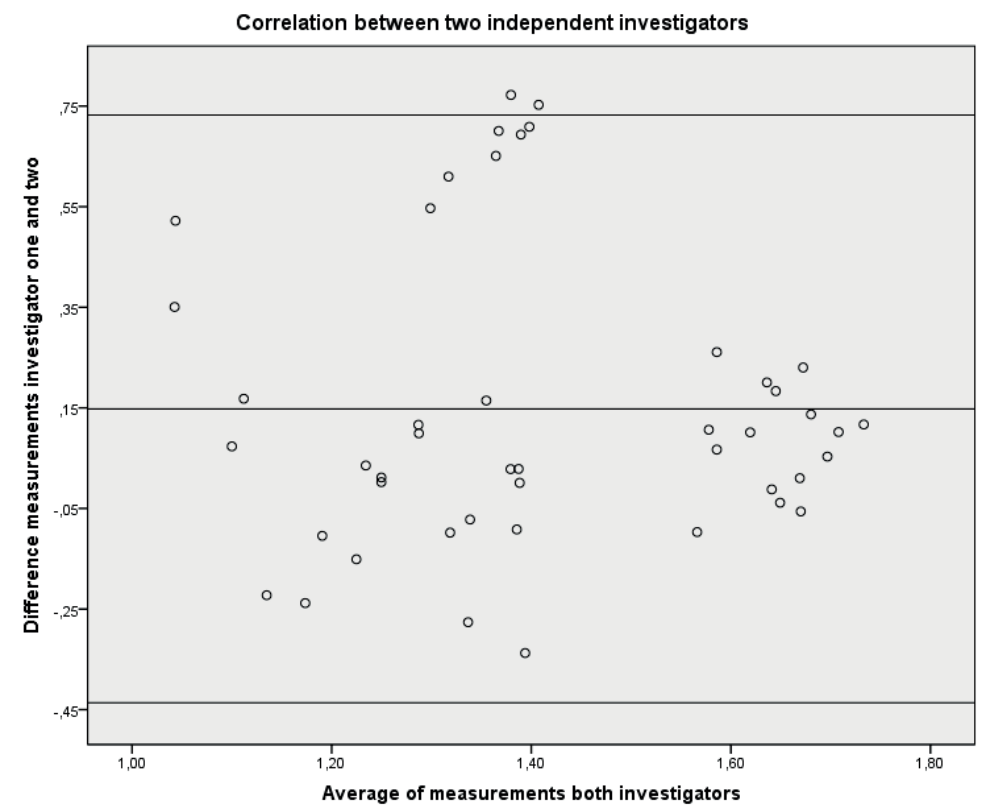

Figure 3. Bland-Altman limits of agreement plot showing the agreement between the measurements of the 2 investigators. The standard deviation +2 and -2 was calculated, and the differences between both measurements were plotted.

\section{DISCUSSION}

The purpose of the present study was to introduce a large animal model to investigate BMD changes in the mandibles of Göttingen Minipigs irradiated with various doses. Furthermore, our purpose was to investigate a possible correlation between the BMD changes and radiation dose, with and without surgical intervention. Our hypothesis was that we expected a small increase in the BMDQ after irradiation. The specific aim of the present study was to develop a tool to measure the quality of the irradiated bone. We succeeded in defining a large animal model to simulate the tissue reactions induced by various radiation doses in the mandible. At 6 months after irradiation, a significant decrease had occurred in the BMDQ compared with that in the non-irradiated animals, independent of surgical intervention. However, the BMDQ changes did not correlate with the individual radiation doses applied, making a translational correlation from the results of this experiment difficult. To the best of our knowledge, we report the first experimental animal study regarding the effects of various radiation doses on BMD. Our hypothesis stated that an increase in the BMD would occur because of the effects of irradiation on bone. ${ }^{9}$ However, in our experiment, an evident decrease was found, and 
we did not find a significant correlation between the radiation dose and the BMDQ. On both the surgically altered left side and the non-surgically altered right side of the mandible, the bone had a significant decrease in the BMDQ. Because no surgical intervention had occurred in the right mandibular side, these results prove a general loss of bone density after irradiation of the mandible, regardless of any additional surgical intervention. In contrast to our findings, Verdonck et al. found an insignificant increase in the BMDQ after irradiation. ${ }^{9}$ In their study of Göttingen Minipigs, all the molars and premolars were removed. The pigs were allowed to heal for 3 months before irradiation (Cobalt ${ }^{60}, 3$ weekly fractions of $8 \mathrm{~Gy}$ ). CT scans were performed 3 months after irradiation. We believe that in that study's duration, reparatory mechanisms could have caused the BMDQ to increase slightly. Their results can be interpreted as a mechanism seen, for instance, in fractures of the femoral head in which, in adjacent bone, the density will have increased 3 months after a fracture has been treated. After a certain period, the BMD will then return to normal. ${ }^{15}$ One must also accept that despite surgical intervention, animals never rest their mandibles. Reparation and wound healing always occur under physiologic load. This phenomenon could be another explanation for the findings of Verdonck et al. ${ }^{9}$ It is possible that the post-trauma remodelling was partially arrested by irradiation, causing the relatively mild increase in BMD. In accordance with our experimental results, Chen et al. found no changes in BMD in a clinical setting. ${ }^{16}$ Several years after therapeutic pelvic irradiation, they compared the BMD in irradiated $L 5$ vertebrae with the non-irradiated $L 2$ vertebrae of the same individuals using dual-energy x-ray absorptiometry. ${ }^{16}$ Several explanations are possible for the mechanism of this seemingly paradoxical maintenance or even increase in BMD after irradiation. Some investigators believe that a greater attenuation occurs in the bone resorbing osteoclasts compared with the osteoblasts, causing a net increase of bone apposition, which is interpreted as hypermineralization. ${ }^{17}$ Other investigators have described an inflammatory response to irradiation, causing a sclerotic effect on bone density despite the pathophysiologic proof of hypocellularity induced by RT. ${ }^{9,18}$ In their experiment on irradiation and distraction osteogenesis, Fregene et al. found a significant increase in low-mineralized, immature bone and a significant decrease in highly mineralized, mature bone in the irradiated bony callus. ${ }^{11}$ Overall, the densitometric values were unchanged. The irradiation induced a diminution in cell function, impairing optimal bone regeneration and resulting in growth of dens of unripe bone. Because of these mineralization pattern variations, it was difficult to detect impaired bone regeneration capacity using only BMD measurements using large bone volume test methods. ${ }^{11}$ The decrease in BMD found in our experiment compared with the insignificant increase found in the same animal species under similar experimental conditions used by Verdonck et al. requires an explanation. ${ }^{9}$ We believe that the differences in BMD measurements could have resulted from measuring different locations in the mandible and, possibly, the presence of some low mineralized immature bone as a reaction to the irradiation. Additionally, the variable timing of tooth extractions and implant placement can help to explain this difference. Finally, the different irradiation 
pattern of applying cobalt ${ }^{60}$ in 3 weekly fractions of 8 Gy might help to explain the difference. We believe that our irradiation schedule, based on a realistic high-dose photon irradiation protocol, resembled the clinical reality better than the scheme used by Verdonck et al.. ${ }^{9}$ The clinical reaction to irradiation of the test animals was close to the acute and chronic tissue reactions observed in irradiated patients with head and neck cancer. The formation of radiation-induced bone sequestration in 2 pigs supports our hypothesis that the experimental setting presented can be used as a large animal model for the evaluation of RT-induced tissue changes. The definition of RT-related risks for the development of ORN is impossible from the data we have presented, because a direct correlation between the radiation doses applied and the changes in BMD could not be proved. The number of pigs per group was relatively small, but the decrease in the BMDQ in general was still significant. The radiation doses given were clinically relevant, because 2 pigs developed ORN at dose levels known to cause ORN. ${ }^{1-3}$

Evaluating the measurements, we found a wide dispersion of the points. We interpreted this finding as inevitable variation, because it was impossible for the 2 independent investigators to measure exactly in the same location on the CT scans. In addition, the size of the measured area varied from 5 to $10 \mathrm{~mm}$. Despite these challenges, it is absolutely possible to perform reproducible measurements in this manner. The methods used in our experiment were reproducible and precise enough to detect significant changes in the BMDQ after irradiation. We did not observe scattering from the titanium implants. Because the gantry was oriented coronally instead of axially (such as it is for humans), the problem of scattering was greatly reduced (Fig 1). The major drawback was that we could not prove significant BMDQ changes in relation to the radiation dose applied. A possible significant relation between the radiation dose and BMDQ might be achieved with larger groups.

The changes in the BMD measured using the BMDQ mirrors the effect of the pathophysiologic reactions of the bone tissue to irradiation on the bone matrix mineral content. Because some studies have found an increase in BMD after irradiation and others have found a decrease in BMD, we are still not sure which part of these pathophysiologic reactions is responsible for BMD changes nor of the clinical consequences.

In conclusion, we have succeeded in defining a large animal model to simulate the tissue reactions induced by various radiation doses in the mandible. At 6 months after irradiation, a significant decrease in the BMDQ compared with the non-irradiated pigs was found, independent of surgical intervention. However, the BMDQ changes did not correlate with the individual radiation doses applied, making a translational correlation from the results of this experiment difficult. Future research should aim to investigate the pathophysiologic reactions of bone tissue after irradiation and imaging or measuring these reactions of the bone tissue, for instance, with magnetic resonance imaging of the bone marrow. 


\section{REFERENCES}

1. Teng MS, Futran ND: Osteoradionecrosis of the mandible. Curr Opin Otolaryngol Head Neck Surg 13:217, 2005

2. Jereczek-Fossa BA, Orecchia R: Radiotherapy-induced mandibular bone complications. Cancer Treat Rev 28:65, 2002

3. Lyons A, Ghazali N: Osteoradionecrosis of the jaws: Current understanding of its pathophysiology and treatment. Br J Oral Maxillofac Surg 46:653, 2008

4. Wildermuth O, Cantril ST: Radiation necrosis of the mandible. Radiology 61:771, 1953

5. Delanian S, Depondt J, Lefaix JL: Major healing of refractory mandible osteoradionecrosis after treatment combining pentoxifylline and tocopherol: A phase II trial. Head Neck 27:114, 2005

6. Marx RE: Osteoradionecrosis: A new concept of its pathophysiology. J Oral Maxillofac Surg 41:283, 1983

7. Marx RE, Johnson RP: Studies in the radiobiology of osteoradionecrosis and their clinical significance. Oral Surg Oral Med Oral Pathol 64:379, 1987

8. Delanian S, Lefaix JL: The radiation-induced fibroatrophic process: Therapeutic perspective via the antioxidant pathway. Radiother Oncol 72:119, 2004

9. Verdonck HW, Meijer GJ, Nieman FH, Stoll C, Riediger D and de Baat C: Quantitative computed tomography bone mineral density measurements in irradiated and non-irradiated minipig alveolar bone: An experimental study. Clin Oral Implants Res 19:465, 2008

10. Shao Z, Liu B, Liu Y, Liu W, Liu R, Peng Q, Liu L: Distraction osteogenesis in the irradiated rabbit mandible. J Plast Reconstr Aesthet Surg 59:181, 2006

11. Fregene $A$, Jing $X L$, Monson LA, Buchman SR: Alteration in volumetric bone mineralization density gradation patterns in mandibular distraction osteogenesis following radiation therapy. Plast Reconstr Surg 124:1237, 2009

12. O'Donovan DA, Yeung I, Zeman V, Neligan PC, Pang CY, Forrest CR: Radiation-induced craniofacial bone growth inhibition: Development of an animal model. J Craniofac Surg 12:533, 2001

13. Todisco M, Trisi P: Bone mineral density and bone histomorphometry are statistically related. Int J Oral Maxillofac Implants 20: 898, 2005

14. Bland JM, Altman DG: Statistical methods for assessing agreement between two methods of clinical measurement. Lancet 1:307, 1986

15. lida $\mathrm{Y}$, Kuroda $\mathrm{T}$, Kitano $\mathrm{T}$, Mizuno K: Dexa-measured bone density changes over time after intertrochanteric hip fractures. Kobe J Med Sci 46:1, 2000

16. Chen HH, Lee BF, Guo HR, Su WR, Chiu NT: Changes in bone mineral density of lumbar spine after pelvic radiotherapy. Radiother Oncol 62: 239, 2002

17. Margulies B, Morgan H, Allen M, Strauss J, Spadaro J, Damron T: Transiently increased bone density after irradiation and the radioprotectant drug amifostine in a rat model. Am J Clin Oncol 26:e106, 2003

18. Williams HJ, Davies AM: The effect of x-rays on bone: A pictorial review. Eur Radiol 16:619, 2006 


\section{CHAPTER 5}

Radiological changes with MR-imaging and CT after irradiating minipig mandibles. The role of T2-SPIR mixed signal intensities in the detection of osteoradionecrosis. 


\section{ABSTRACT}

Background Radiotherapy in the head and neck can induce several radiologically detectable changes in bone, osteoradionecrosis (ORN) among them. However, until now only a few studies on typical radiological changes exist.

Methods Sixteen Göttingen minipigs were irradiated with equivalent doses of 0, 25, 50 and $70 \mathrm{~Gy}$. Three months after irradiation, left mandibular teeth were removed and dental implants were placed. CT-scans and MR-imaging were made before irradiation and six months after. Alterations in the bony structures were recorded on CT-scan and MR-imaging.

Results Higher radiation dose were associated with increased signal changes on MRimaging. On CT-scans cortical destruction was found in three animals. Based on imaging, three animals were diagnosed with ORN.

Conclusion Whereas clinically only exposed bone can be appreciated in ORN, CT and MR-imaging facilitates visualisation of skelettal changes, thus adding to the accuracy and early detection of beginning or latent ORN. 


\section{INTRODUCTION}

Treatment of head and neck malignancies frequently includes radiotherapy (RT) which has been proven to have beneficial effects on locoregional recurrence and survival. ${ }^{1,2}$ However, despite improved radiotherapeutical techniques, inevitable irradiation effects on the surrounding healthy tissue are still a concern in terms of complications. ${ }^{3,4}$

Osteoradionecrosis (ORN) occurs especially in the mandible and is considered a serious complication of RT. ORN can be triggered by surgery or occur spontaneously. It can arise at any moment after RT, even after several years. If conservative treatment of ORN fails, radical resection and micro-vascular reconstruction is indicated, requiring a major surgical intervention. 5

The most commonly used definition of ORN is based on clinical presentation and observation; "irradiated bone becomes devitalized and is no longer covered by skin or mucosa without healing for 3 months, without recurrence of tumor". 7 The reported incidence of ORN in literature ranges between 2 and $22 \%$ for the mandible. ${ }^{8}$ Several factors that can increase the risk of developing ORN have been identified, and include for instance age, sex, general health, smoking, dose and volume of irrradiation. 8 Marx and Johnson discovered a double peak in occurrence at 1 year and approximately 4 years after irradiation. ${ }^{9}$ Several classifications for ORN exist, the most practical being the Notani classification: Class I ORN is confined to dentoalveolar bone, class II ORN is limited to dentoalveolar bone or mandible above the inferior dental canal, or both and class III ORN is characterized by affection of either the mandible below the inferior dental canal, or pathological fracture, or skin fistula. ${ }^{10}$

The susceptibility of the mandible to develop ORN is mainly the result of RT induced fibrosis of the bone marrow that causes obliteration of the inferior alveolar artery. As the facial artery is incapable to supply sufficient collateral blood supply, ORN occurs. ${ }^{11}$

\section{Imaging findings}

To the best of our knowledge there are only few studies on MR-imaging changes in the mandible after irradiation yet. ${ }^{12-14}$ Known MR-imaging changes of bone marrow in vertebrae and the femur head after irradiation consist of decreased signal intensity on T1-weighted (T1), and T2-weighted (T2) images may show mixed signal intensity corresponding to areas of bone marrow edema pattern (BMEP) and fibrosis after 6 weeks and onwards. With chronicity, focal areas of fat may be deposited, which on T1 images will appear as areas of increased signal intensity. Fibrosis and areas of sclerosis are characterized by hypointense signal on both T1 and T2 images. ${ }^{15,16}$

Bachman et al. described MR-imaging findings of the mandible after RT. They state that tooth extraction in or resection of irradiated mandibular bone causes localized edema 
of the alveoli or resection margins. However, periodontal disease in irradiated bone causes only minimal BMEP or no signal abnormalities at all on MR-imaging. ${ }^{12}$ Further, they conclude that MR-imaging allows for detection of pathologic changes of mandibular bone marrow in ORN. The inflammatory process is diffusely disseminated in the mandible, often not respecting the cortical boundaries and invading paraossal tissue. Signal characteristics of BMEP on MR-imaging consist of reduced signal intensity on T1 images and increased signal intensity on T2 images. ${ }^{12}$ Store et al. found that several years after RT, no difference in signal intensity between irradiated and nonirradiated, normal mandibular bone was visible anymore. ${ }^{13}$

Typical CT-scan findings of ORN in literature consist of cortical disruption with disorganization and loss of trabeculation of the spongiosa of the mandible. ${ }^{17}$ Cortical bone fragmentation is present in most cases. ${ }^{17}$ In more severe cases, pathologic fracture can occur. ${ }^{18}$ Cortical thickening may be present. ${ }^{16}$

These abnormalities are predominantly seen in the body of the mandible (premolar and molar region). In some cases even extension into the mandibular angle or retromolar triangle can be found. ${ }^{19}$

Purpose of this study: Since there is only limited literature available on RT induced structural changes in the mandible, the aim of our study was to describe imaging findings in mandibular bone after various doses of irradiation with and without surgery in order to identify typical imaging characteristics of post-irradiation changes and ORN.

\section{MATERIALS AND METHODS}

\section{Research design}

In this animal study 16 healthy adult 18 month-old female Göttingen minipigs were used (weight 35-45 kilogram). The study was performed in accordance with the Dutch and European Community guidelines for the protection of (laboratory) animals and partly described in our earlier publication. ${ }^{20}$ Permission was obtained from the local Animal Ethical Committee (DEC 2011-127).

\section{Procedures}

After initial baseline imaging, consisting of CT- scan and MR-imaging, RT as described below took place. 3 months later, surgery was performed. At 6 months after RT, imaging was repeated, using MR-imaging protocols identical with the baseline scans. 


\section{Imaging}

For irradiation planning purposes a CT-scan (Siemens Sensation Open, 120kV, 270mAs, $\mathrm{CDTI} /$ vol 29,32 mGy, 0,6mm slices, FOV 500mm, Matrix 512x512, Siemens, Erlangen, Germany) was performed under general anesthesia using an individualised thermoplastic immobilisation mask for optimal fixation of the animal, made immediately before scanning. The CT-scan was directly followed by a $1,5 \mathrm{~T}$ MR-scan $(1,5$ Tesla Intera, Phillips Healthcare, Best, the Netherlands), still under anesthesia but without the immobilisation mask. Six months after irradiation, imaging with CT (Siemens Somatom Definition Flash, Dual Scource, Dual Energy tube A Sn140kV, 225mAs, tube B $80 \mathrm{kV}$ - $450 \mathrm{mAs}, \mathrm{CDTI}$ vol 38,15 mGy , 0,6mm slices, FOV 300mm, Matrix 512x512, Siemens Erlangen, Germany) and MR-scans were repeated again under general anesthesia. Immediately after imaging the animals were sacrificed. The MR-imaging protocol consisted of coronal T1, T2 and T2-SPIR, transverse STIR images and coronal post-gadolinium T1 images of the mandible using a head-coil. (T1 sequence, TR/TE=530/11ms, T2 sequence, TR/TE=3600/80ms, T2-SPIR, TR/TE=3700/80ms, STIR, $\mathrm{TR} / \mathrm{TE}=3900 / 15 \mathrm{~ms}$, T1 sequence after gadolinium (Gadobutrol 1,0mmol/ml; 7,5ml i.v., $0,2 \mathrm{mmol} / \mathrm{kg}$ ), TR/TE 470/15ms, FOV 220-240mm and slice thickness 3.0mm).

\section{Irradiation}

Four animals each were assigned to one of the four groups receiving an equivalent dose (EQD) of 0, 25, 50 or 70 Gray (Gy) respectively. Irradiation took place under general anesthesia, one week after imaging. For optimal positioning, the animals were immobilized using an individualized thermoplastic mask for the treatment planning CTscan as well as the radiation fractions. For irradiation, a 6 megavolt linear accelerator (Linac Siemens, Erlangen, Germany) with two opposing lateral beams was used. The entire body of the mandible was irradiated in two fractions with an equivalent dose of $0,25,50$ or $70 \mathrm{~Gy}$ (two fractions of $0,6.5,9.7$, and $11.8 \mathrm{~Gy}$ ). In order to calculate the equivalent dose, an alpha/beta ratio of 3 was used for late responding tissues using the linear quadratic formula. ${ }^{21}$ The second fraction was given 48 hours after the first fraction.

\section{Surgical intervention}

Thirteen weeks after irradiation, surgery was performed under general anesthesia.

The surgical intervention on the mandible involved extraction of all premolars and molars on the left side only. In the same session four submerged dental implants, diameter 4,0 $\mathrm{mm}$ and length $12 \mathrm{~mm}$, were placed in the left mandible (BioComp Industries BV, Vught, The Netherlands). The animals received antibiotics (Amoxicillin, AST beheer BV Oudewater, the Netherlands) and pain medication (Temgesic, Reckitt 


\section{CHAPTER 5}

Benckiser Healthcare Hull, United Kingdom), all intramuscularly, which was continued for three days after surgery.

\section{Data analyses}

At the end of the experiment central and cortical destruction and location and presence of implants were described on CT-scan. MR-imaging signal intensities on all sequences were scored, as well as enhancement and cortical destruction (see table 1). Based on this data, presence of ORN was diagnosed or ruled out, respectively. All images were scored by two experienced head-neck radiologists (AP, AS) independently and in consensus, blinded for applied irradiation dose and clinical symptoms.

The gold standard for the diagnosis of ORN was clinical examination revealing persistent intra-oral exposed bone at the time of sacrifice. ${ }^{7}$

The data were transferred to an electronic sheet (Excel ${ }^{\mathrm{TM}}$, Microsoft Office 2010, Redmond, WA, USA), and then processed using SPSS 20 ${ }^{\mathrm{TM}}$ (Statistical Package for the Social Sciences, SPSS Inc., Chicago, IL, USA).

Table 1. Scoring system to define MR-imaging signal intensities.

\begin{tabular}{ll}
\hline Score & definition \\
\hline 4 & mixed signal intensity changes \\
3 & major increase in signal intensity \\
2 & moderate increase in signal intensity \\
1 & minor increase in signal intensity \\
0 & no abnormalities \\
-1 & minor decrease in signal intensity \\
-2 & moderate decrease in signal intensity \\
-3 & major decrease in signal intensity \\
\hline
\end{tabular}

\section{RESULTS}

\section{Clinical outcome}

Two animals had clinical signs of ORN at 6 months after irradiation; one animal in the 50 Gy EQD and one in the 70 Gy EQD group, both left-sided, corresponding to the side of surgical intervention. The area of exposed bone in the animal treated with $70 \mathrm{~Gy}$ measured approximately $5 \mathrm{~cm}$, elevated from the surrounding mucosa and adjacent to two dental implants which were loose, Notani class I (figure 1).

The animal treated with 50 Gy showed exposed bone which was not elevated and was approximately $2 \mathrm{~cm}$ long. Also, there was presence of a submandibular fistula on the left side, Notani class III. 


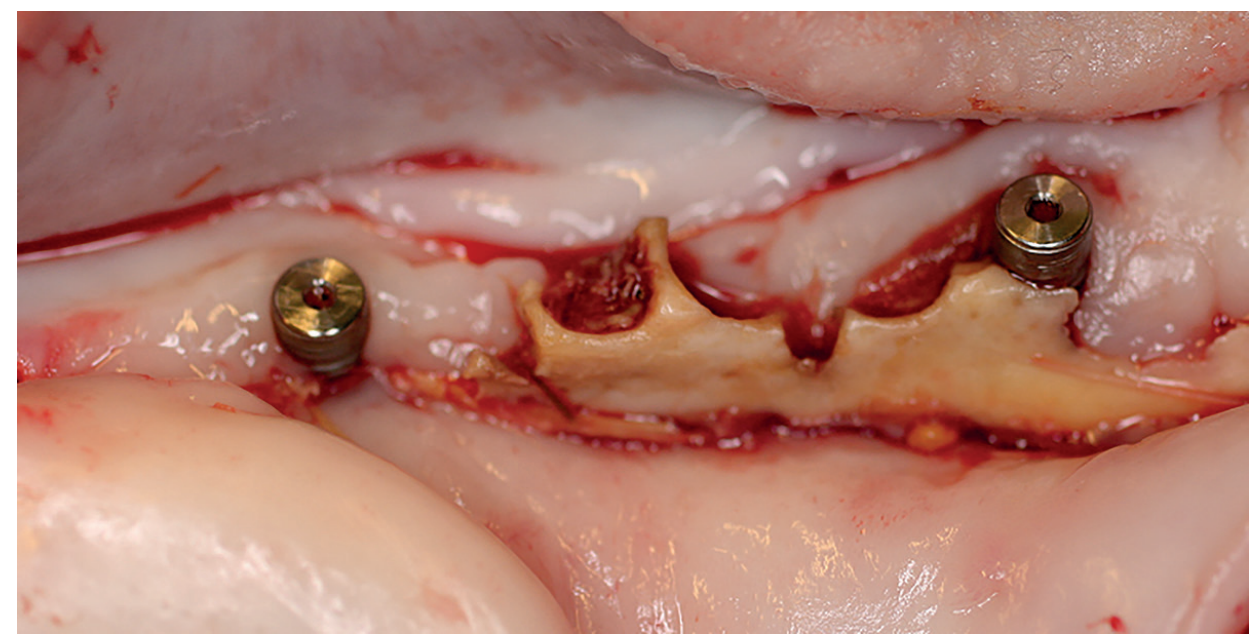

Figure 1. Clinical sign of osteoradionecrosis: exposed bone sequester and mobile dental implants (animal number 15 , dose group of 70 Gray)

\section{$M R I$ and radiation dose.}

At higher radiation doses, signal intensities changed at the operated (left) and nonoperated (right) side (table 2). With increased radiation dose, T1 images depicted a decrease in signal intensity, whereas the signal on STIR and T2-SPIR sequences became hyperintense. On the T1 sequence post gadolinium (CE-T1), the enhancement was more prominent in the animals receiving higher radiation doses. In general, in our study the signal changes were more pronounced with increase of the administered radiation dose, even though there was some overlap.

The animals with clinical ORN demonstrated mixed signal intensities on T2-SPIR on both the left and the right side. The T2 sequences at the right, not operated side demonstrated an iso-intense aspect of the bone marrow at lower radiation doses, changing to mixed signal intensities after irradiation with high radiation doses.

On the operated left side, the signal intensities on T2 images after irradiation were isointense, slightly hyper- or hypointense, at lower radiation doses, with a mixed aspect of signal at higher radiation dose. 


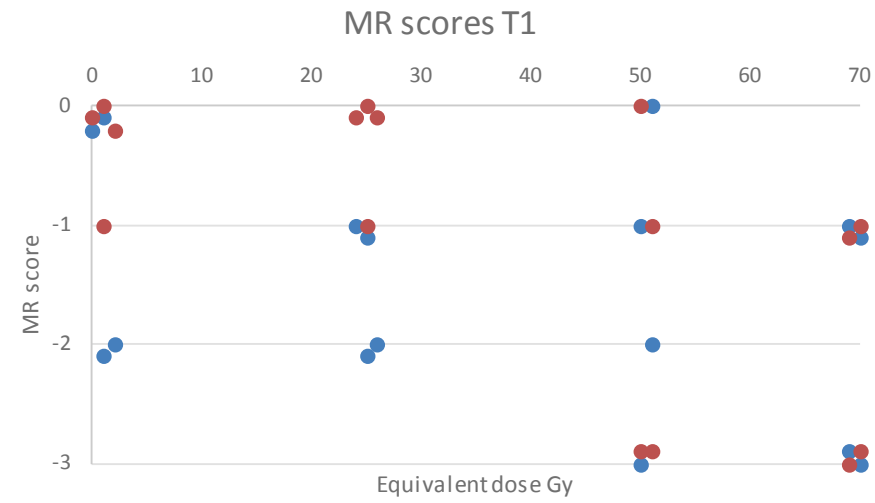

a.

MR scores T2-SPIR

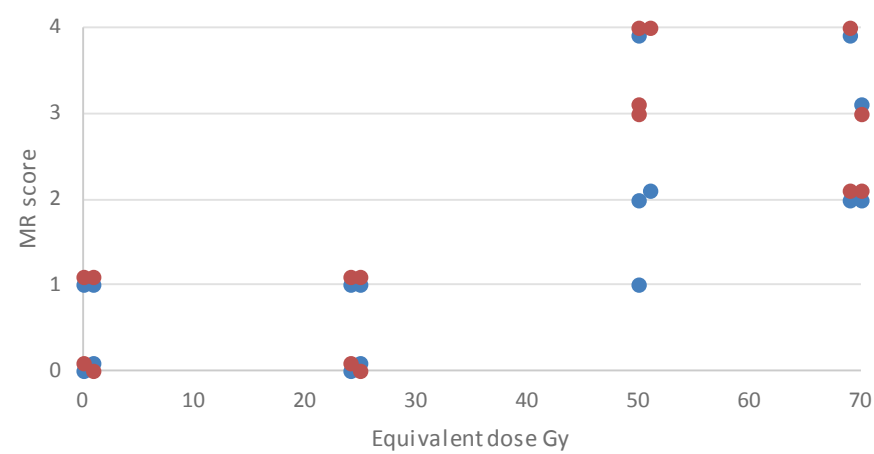

b.

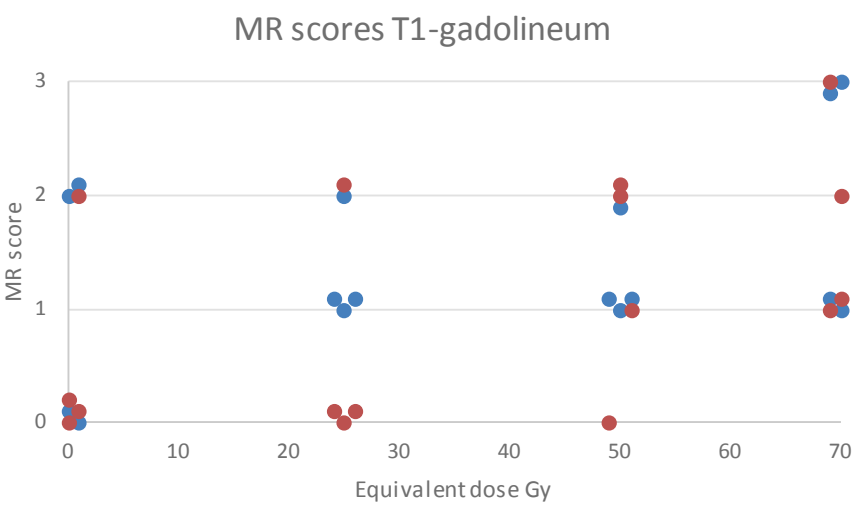

c.

Table 2a, b, and c. MR-imaging scores of the left (blue dots) and right (red dots) mandible, six months post-irradiation in T1-, T2-SPIR-, and T1-weighted sequences after gadolinium. Number of dots given corresponds with number of animals. Scores given in Table 1. 


\section{Cortical destruction}

On the CT-scans performed 6 months after irradiation, cortical bone defects with destruction were seen in three animals. The same three animals were found to have cortical interruption on MR-images after radiotherapy as well. Thus, there was no discrepancy between the two imaging modalities concerning cortical destruction was found. Rather subtle superficial cortical erosion was seen on CT in two other animals, not visible on MR-imaging. Viseversa, there were no cortical changes on MR-imaging which could not be found on CT. Two animals had a slight cortical thickening on CT due to dental infections. One animal in the 50 Gy group demonstrated a cortical defect, which was already present on the baseline images due to pre-existent abscess and sequestration.

\section{Imaging findings in clinical ORN}

In the two animals with clinical signs of ORN, the baseline CT-scans were normal.

On the scans at 6 months after irradiation, the EQD 50Gy ORN animal showed a minor bone sequester on the left side and bilateral cortical destruction with substantial thickening of the mandibular cortex after irradiation.

In the EQD 70Gy ORN animal, a large bone sequester and cortical destruction on the left mandible were seen (figure 2).

On the initial MR-images, the EQD 50Gy ORN animal was found to have moderately decreased bone marrow signal intensity on T1 images and moderate to major increased signal intensity on STIR and T2-SPIR. There was enhancement on the CE-T1. The followup imaging after irradiation and surgery showed no change in signal intensity on STIR and CE-T1 sequences. On T2 and T2-SPIR, however, the signal intensity of the bone marrow changed to mixed intensity bilaterally. A left-sided submandibular skin fistula also was present (figure 2).

In the EQD 70Gy ORN animal, on the baseline MR-imaging normal signal intensity of bone marrow was present bilaterally on al sequences. After irradiation and surgery, there was a significant decrease of signal on the T1 images, an increase on STIR with enhancement on CE-T1. As in the other case, signal intensity on T2 and T2-SPIR had changed into mixed intensity bilaterally (figure 2).

\section{Radiological versus clinical ORN}

Based on imaging, the radiologists diagnosed ORN in three different cases. In two cases, radiological findings paralleled the clinical diagnosis of ORN. In the third case, the radiological findings were consistent with ORN, whereas clinically no exposed bone was seen (figure 2). Histological evaluation, however, showed extensive radiation induced damage in this case as well. 


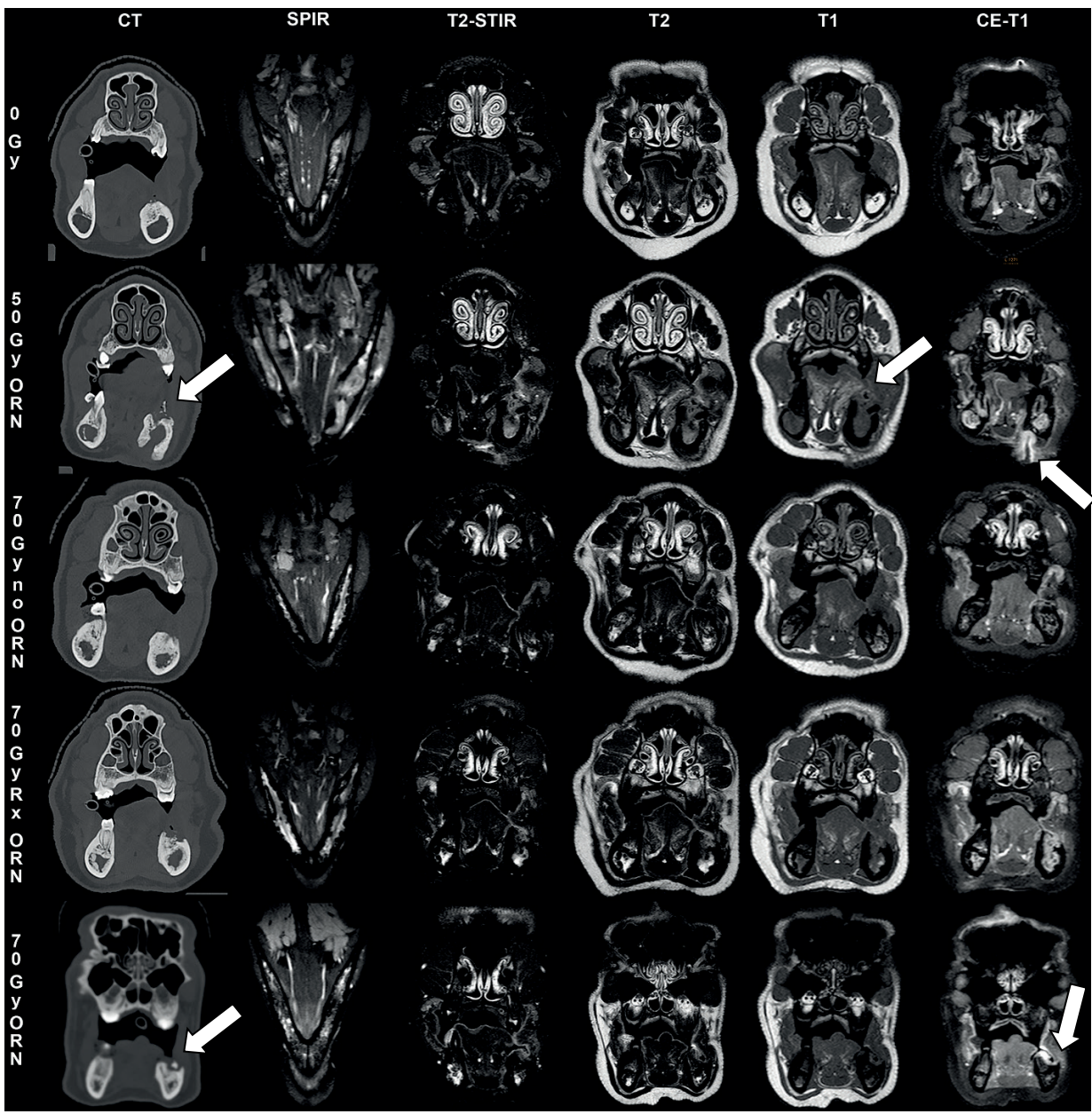

Figure 2. Overview of CT-scan and MR-imaging of the animals number $04,09,16,14$, and 15 . First column are CT-scan images of the animals, note the cortical destruction and sequester of animals 09 and 15 (arrows).

Second until sixth column are MR-images with the SPIR, T2-STIR, T2, T1, and CE-T1 sequences. The first row shows animal number 04, without irradiation; all marrow signal intensities are normal.

The second row shows animal number 09 with decreased signal intensity of the marrow on T1 sequence and sequester on the left side and increase signal in SPIR sequence, mixed signal intensity on T2 and T2-STIR sequences and enhancement on CE-T1 sequence. A fistula (arrow) is clearly visible (Notani grade III).

The third row shows MR-images of animal number 16 without clinical or radiological ORN. Note the minor decrease in signal intensity of the marrow on T1 sequence, minor enhancement after gadolinium, mixed signal intensities on T2 sequence and mixed, moderate to major increased signal intensities on STIR at the mandible right, T2-SPIR and STIR mandible left respectively.

The fourth row shows MR-images of animal number 14, radiological ORN without clinical signs. Note the decrease of signal intensity of the marrow on T1 sequence and increase signal in SPIR and T2 STIR sequences, with mixed signal intensity on T2-weighted sequence and enhancement on CE-T1 sequence.

The fifth row shows MR-images of ORN, animal number 15 . Note the decrease in signal intensity of the marrow on T1 sequence and increase signal on SPIR sequence, mixed signal intensity on T2 and T2-STIR sequences and enhancement on CE-T1 sequence. The sequester is marked with an arrow (Notani grade I). 


\section{DISCUSSION}

To our knowledge, this was the first large animal experiment simulating radiotherapy induced changes of bone in head and neck based on the current treatment regime of patients with head and neck malignancies.

Through the course of the experiment, two animals, i.e. 2 of 12 animals irradiated gives an incidence of $17 \%$, developed clinical ORN. As this is a serious complication of radiotherapy, we consider our study as a relevant animal model for RT induced changes in the head and neck region.

The CT-scan findings in ORN with cortical destruction, disorganization and loss of trabeculation of the mandibular spongiosa were the same in our animal model as in a human patient population. ${ }^{17}$ Whereas patients with ORN frequently present with a pathologic fracture of the mandible, we did not encounter fractures in our cohort, probably due to the relative short follow-up period. ${ }^{19}$ In one of the two animals presenting with clinical and radiological ORN next to a substantial reactive subperiostal bone apposition on $\mathrm{CT}$ there was presence of a skin fistula detected on MR-imaging.

In humans, evident periosteal reactions and soft tissue pathologies are distinctly uncommon in the uncomplicated case of ORN but may occur when complicated by infection or fracture. ${ }^{16}$ Since there was no fracture present in our animal study, we suppose this reactive subperiosteal bone apposition to be due to concomitant infection of damaged irradiated bone with reparative periosteal bone formation.

Interestingly there was no discrepancy between CT-scan and MR-imaging findings on cortical bone destruction. If no metal artefacts caused by dental fillings, prosthetic work or dental implants are present, MR-imaging has thus the potential of detection of cortical destruction without additional CT-scan. Unfortunately, these metal components are quite frequent in human population. Furthermore, erosions and trabecular destruction are depicted on CT in greater detail and maybe at an earlier state, whereas MR-imaging is superior in detecting even early bone marrow changes such as fibrosis and edema.

One animal in the EQD 70Gy and one in the EQD 50 Gy group were diagnosed clinically as well as radiologically with ORN. One more animal in the EQD 70Gy group met the radiological criteria for ORN but did not have exposed bone resulting in a discrepancy between the clinical and radiological findings. Histology, however, showed indeed extensive bone damage. As the bone might only get exposed at a later stage of ORN and consequently will be clinically diagnosed later, as such an underreporting of ORN might be the result. ${ }^{12}$ The late recognition of ORN may result in spontaneous or post-surgical complications. This emphasizes the need for early visualization of RT induced damage to the bone. 
This is supported by Bachman et al. who promotes the use of MR-imaging for early detection of bone marrow changes to enable the diagnosis of mandibular ORN before clinical symptoms occur. ${ }^{12}$ Store et al. described MR-imaging findings in patients with ORN with and without bone exposure. ${ }^{13}$ In their collective, the patients without bone exposure at the time of examination did have exposed bone earlier on, which healed with conservative treatment. They concluded that MR-imaging revealed the still active pathological process whilst the overlying tissues had already healed. So healing of the intraoral defect is not a sign of complete restitution.

Reconsidering the radiological and clinical data in our study, we have to conclude that there were indeed three animals with ORN, albeit in different stadia.

The animal in the EQD 50 Gy group diagnosed with ORN had bone marrow intensity changes on the pre-irradiation images possibly related to dental problems. This could have predisposed to the development of ORN. On the other hand another animal in the 50 Gy group had a dental abscess with cortical destruction pre-irradiation and did not develop ORN.

T2-SPIR sequences with mixed signal intensities after irradiation were exclusively found in the two cases of ORN with matching clinical and radiological diagnosis; however, the signal changes were seen bilaterally whereas clinical ORN was confined to the left operated-on side.

Inflammation is the initial reaction upon RT and causes edema of the irradiated bone, edema being hyperintense on T2-SPIR. As RT can also induces fibrous tissue formation, which is hypointense on T2-SPIR, in a later stage, the mixed signal intensity could represent the presence of already fibrotic areas within areas of edema. ${ }^{22}$ Interestingly, the animal with radiological, but no clinical signs of ORN did not show mixed signal intensities on T2-SPIR. ORN still in the initial phase - with edema diffusely throughout the bone marrow and almost no fibrous tissue yet - could be a possible explanation.

As matching radiological and clinical findings of ORN were only seen on the left mandibular side, a relation between surgical intervention and the development of ORN seems to be likely. ${ }^{7,22}$ However, on MR-images the same changed signal intensities were seen in the right, non-operated on side of the mandible.

Also, the other irradiated animals, showed with increase in irradiation dose a progressive decrease in bone marrow signal intensities on the T1 images, and signal increase on the T2 images, as well as enhancement on CE-T1 images. Signal increase on T2 images indicates edema. This edema might be caused by the prolonged process of regenerative repair of irradiated bone and surrounding tissues. A visible increase in edema was related to an increased irradiation dose. According to the literature this process will stabilize over time after completion of RT. Finally, one can expect the bone marrow signal will return to normal. ${ }^{13}$ The cortical destruction with or without 
sequestration seen in ORN might be the result of unrestrained or overactive resorption by osteoclasts in the regenerative phase after RT as described by Store et al. ${ }^{13}$ Possibly this osteoclastic "overactivity" is the underlying pathophysiological process in development of ORN. However, the reasons for insufficient regenerative repair escalating into ORN remain unclear, just as the question why not all the animals treated with higher doses developed radiological or clinical ORN.

This study suffers from several limitations. Firstly, due to ethical considerations, the number of animals was limited. Secondly, irradiation was administered in only two fractions for ethical and logistical reasons. In humans, fractionated irradiation is applied, according to evidence-based treatment protocols, in high fractionation or even hyperfractionation. ${ }^{1,2,4}$ However based on the linear quadratic formula we have attempted to administer doses similar to the fractionated schedules used in humans. Thirdly, the observation period after radiotherapy was only 6 months. It is known, however, that ORN can develop even years after RT. ${ }^{7,22}$ The restricted possibility of thorough clinical exploration and inspection is another limitation; due to anesthesia and the risks of sedation, a detailed clinical examination was facilitated only after the sacrifice of the animals. An exact evaluation of duration of clinical ORN was therefore not possible.

Establishing more precise methods to evaluate bony alterations, using MR- and CTimaging, following RT for early detection of ORN as well as possibly to identify patients prone to develop ORN even prior to radiotherapy are subject to future research.

\section{CONCLUSION}

Six months after RT, MR-imaging of irradiated mandibulae showed increasing abnormal signal changes of bone marrow with increasing radiation dose. This phenomenon could be detected as well on the non-operated as the operated side of the mandible. These signal changes can be interpreted as signs of a prolonged regenerative repair following bone damage induced by RT.

Mixed signal intensities on T2-SPIR after RT were exclusively found in the clinical ORN cases and visualize the pathophysiological process closely related to development of ORN. Therefore, T2-SPIR sequence should be included in any MR-imaging protocol for ORN. Whereas the clinical diagnose of ORN can only be made on grounds of macroscopic evidence of failed osseous regeneration after RT, CT-scans and MRimaging allow an early detection of pathologic changes consistent with ORN. Therefore, in our opinion an early application of both CT and MR-imaging will be beneficial in diagnosing even subtle signs of early ORN. 


\section{ACKNOWLEDGEMENTS}

We kindly thank BioComp Industries BV, Vught, the Netherlands, for partially funding this animal experiment.

We kindly thank all radiotherapy lab technicians and especially Mrs Granzier for the individualised irradiation planning and organisation. 


\section{REFERENCES}

1. Peters LJ, Goepfert H, Ang KK, Byers RM, Maor MH, Guillamondegui O, Morrison WH, Weber RS, Garden AS, Frankenthaler RA, and et al., Evaluation of the dose for postoperative radiation therapy of head and neck cancer: first report of a prospective randomized trial. Int J Radiat Oncol Biol Phys, 1993. 26(1): p. 3-11.

2. Bourhis J, Overgaard J, Audry H, Ang KK, Saunders M, Bernier J, Horiot JC, Le Maitre A, Pajak TF, Poulsen MG, O'Sullivan B, Dobrowsky W, Hliniak A, Skladowski K, Hay JH, Pinto LH, Fallai C, Fu KK, Sylvester R, and Pignon JP, Hyperfractionated or accelerated radiotherapy in head and neck cancer: a meta-analysis. Lancet, 2006. 368(9538): p. 843-54.

3. Lee J and Moon C, Current status of experimental therapeutics for head and neck cancer. Exp Biol Med (Maywood), 2011. 236(4): p. 375-89.

4. Thariat J, Bolle S, Demizu Y, Marcy PY, Hu Y, Santini J, Bourhis J and Pommier P, New techniques in radiation therapy for head and neck cancer: IMRT, Cyberknife, protons, and carbon ions, Improved effectiveness and safety? Impact on survival? Anticancer Drugs, 2011. 22: p. 596-606.

5. Jereczek-Fossa BA and Orecchia R, Radiotherapy-induced mandibular bone complications. Cancer Treat Rev, 2002. 28(1): p. 65-74.

6. Jacobson $\mathrm{AS}$, Buchbinder $\mathrm{D}, \mathrm{Hu} \mathrm{K}$ and Urken $\mathrm{ML}$, Paradigm shifts in the management of osteoradionecrosis of the mandible. Oral Oncol, 2010. 46(11): p. 795-801.

7. Lyons A and Ghazali N, Osteoradionecrosis of the jaws: current understanding of its pathophysiology and treatment. Br J Oral Maxillofac Surg, 2008. 46(8): p. 653-60.

8. Teng MS and Futran ND, Osteoradionecrosis of the mandible. Curr Opin Otolaryngol Head Neck Surg, 2005. 13(4): p. 217-21.

9. Marx RE and Johnson RP, Studies in the radiobiology of osteoradionecrosis and their clinical significance. Oral Surg Oral Med Oral Pathol, 1987. 64(4): p. 379-90.

10. Notani K, Yamazaki Y, Kitada H, Sakakibara N, Fukuda H, Omori K and Nakamura M, Management of mandibular osteoradionecrosis corresponding to the severity of osteoradionecrosis and the method of radiotherapy. Head Neck, 2003. 25(3): p. 181-6.

11. Zhuang $\mathrm{Q}$, Zhang $\mathrm{Z}$, Fu H, He J and He $\mathrm{Y}$, Does radiation-induced fibrosis have an important role in pathophysiology of the osteoradionecrosis of jaw? Med Hypotheses, 2011. 77(1): p. 63-5.

12. Bachmann G, Rossler R, Klett R, Rau WS and Bauer R, The role of magnetic resonance imaging and scintigraphy in the diagnosis of pathologic changes of the mandible after radiation therapy. Int J Oral Maxillofac Surg, 1996. 25(3): p. 189-95.

13. Store G, Smith HJ and Larheim TA, Dynamic MR imaging of mandibular osteoradionecrosis. Acta Radiol, 2000. 41(1): p. 31-7.

14. Deshpande SS, Thakur MH, Dholam K, Mahajan A, Arya S and Juvekar S, Osteoradionecrosis of the mandible: through a radiologist's eyes. Clin Radiol, 2015. 70(2): p. 197-205.

15. Stevens SK, Moore SG and Kaplan ID, Early and late bone-marrow changes after irradiation: MR evaluation. AJR Am J Roentgenol, 1990. 154(4): p. 745-50.

16. Tartaglino LM, Rao VM and Markiewicz DA, Imaging of radiation changes in the head and neck. Semin Roentgenol, 1994. 29(1): p. 81-91.

17. Hermans R, Fossion E, loannides C, Van den Bogaert W, Ghekiere J and Baert AL, CT findings in osteoradionecrosis of the mandible. Skeletal Radiol, 1996. 25(1): p. 31-6.

18. Fujita M, Harada K, Masaki N, Shimizutani K, Kim SW, Fujita N, Sakurai K, Fuchihata H, Inoue T and Kozuka T, [MR imaging of osteoradionecrosis of the mandibula following radiotherapy for head and neck cancers]. Nihon Igaku Hoshasen Gakkai Zasshi, 1991. 51(8): p. 892-900.

19. Chong J, Hinckley LK and Ginsberg LE, Masticator space abnormalities associated with mandibular osteoradionecrosis: MR and CT findings in five patients. AJNR Am J Neuroradiol, 2000. 21(1): p. 175-8.

20. Poort LJ, Bittermann BG, Böckmann RA, Hoebers FJ, Houben R, Postma AA, Kessler PAWH Is there a change in bone mineral density changes of the mandible in Göttingen Minipigs after irradiation in correlation with radiation dose and implant surgery? J Oral Maxillofac Surg, 2014. 72(11): p. 2149-2156. 
CHAPTER 5

21. Fowler JF, 21 years of biologically effective dose. Br J Radiol, 2010. 83(991): p. 554-68.

22. Marx RE, Osteoradionecrosis: a new concept of its pathophysiology. J Oral Maxillofac Surg, 1983. 41(5): p. 283-8. 


\section{CHAPTER 6}

Dual-Energy CT versus MR imaging for the detection of bone marrow edema in pig mandibles treated with radiotherapy and surgery

L.J. Poort, A.A.R. Stadler, J.H.B. Ludlage, F.J.P. Hoebers, P.A.W.H. Kessler, A.A.Postma Submitted to European Journal of Radiology 


\section{ABSTRACT}

Objective To investigate the accuracy of Dual Energy CT (DE-CT) compared to MR imaging for the detection of edema of the mandible after irradiation and surgery in an osteoradionecrosis animal model .

Materials and methods 15 adult Göttingen minipigs received irradiation to the mandible with an equivalent dose of $0,25,50$ or 70 Gy. At 3 months, tooth extraction and the placement of 4 implants was performed in the left mandible. 6 months after irradiation, all animals underwent DE-CT and MR imaging of the mandible. MR STIR was used for the grading of the bone marrow edema (0-3). Simultaneous imaging with DE-CT at 80 $\mathrm{kVp}$ and $140 \mathrm{kVp}$ was performed, and virtual non-calcium (VNCa) images were calculated. The HU values were measured in 15 pigs by 2 observers and compared to the MR imaging.

Results Increased signal intensity at STIR was found in the higher radiation groups. An increase of signal intensity in STIR imaging was accompanied by a significant increase in the $\mathrm{HU}$ value of the VNCa images of the bone marrow (STIR 0,1,2,3; mean HU -103, -90, $-76,-34$, respectively; $p<0.05$ for $0-3,1-2$ and $2-3) . R^{2}=0.388$.

Conclusion The VNCa images derived from DE-CT are able to demonstrate bone marrow edema in radiation-induced bone changes in the mandible. In addition to the ability of CT to depict cortical destruction and trabecular bone resorption, the DE-CT VNCa images can provide additional information on the presence of bone marrow edema. 


\section{INTRODUCTION}

Osteoradionecrosis (ORN) of the mandible is a late complication of radiotherapy after the treatment of head and neck cancer, with an incidence of $5 \%$ and can occur spontaneously or after surgical intervention. ${ }^{1}$ ORN is diagnosed clinically as the presence of persistent intra-oral exposed bone. ${ }^{2}$ The radiological diagnosis of ORN requires complementary $C T$ and $M R$ imaging. $C T$ depicts the effects on mineralized bone tissues by demonstrating cortical disruption and the loss of trabeculation of the spongiosa. ${ }^{3}$ Generally, the MR imaging findings of ORN of the mandible reflect the process of "inflammation" within the bone as a bone marrow edema pattern, demonstrated by an increased signal intensity in T2-weighted images. ${ }^{4}$

Dual Energy Computer Tomography (DE-CT) allows for the analysis of material composition, by using two image datasets of the same anatomic location with two different $x$-ray spectra. ${ }^{5-7}$ The differences in the mass-attenuation coefficients underlay the ability to characterize and separate tissues using DE-CT. The applications of DE-CT are still increasing and include material characterization as in bone removal and iodine detection and quantification; they are used eg. in CT-angiography and renal masses. ${ }^{8}$ Mono-energetic applications can be used for increased conspicuity of iodine by increasing CNR at lower $\mathrm{kV}$ or higher SNR at high $\mathrm{kV} .{ }^{9}$ More recently virtual non-Calcium imaging (VNCa) was introduced for the detection of (posttraumatic) bone marrow edema in large bones as are the vertebrae, femur and tibia. ${ }^{10-13}$

The application of this VNCa decomposition algorithm with DE-CT is until now not used in head and neck radiology.

We investigated in an existing animal model for ORN whether DE-CT derived VNCa were able to detect BME pattern in the operated and/or irradiated mandible.

\section{Objective}

To investigate the accuracy of DE-CT-derived VNCa images compared to MR imaging for the detection of edema of the mandible after irradiation and surgery in an ORN animal model.

\section{MATERIALS AND METHODS}

This study was part of a larger experimental animal study investigating irradiation effects on the mandible and the development of ORN. ${ }^{14}$ The study was performed in accordance with the Dutch and European Community guidelines for the protection of (laboratory) animals. Permission was obtained from local Animal Ethical Committee (DEC 2011-127). 
As ORN can occur spontaneously after radiotherapy or after radiotherapy by surgical intervention, we chose the following setup:

- A split mouth design was used to study the effect of irradiation with and without surgical intervention.

- The surgical intervention chosen was a typical oral surgical intervention, tooth extraction and implant placement, used for oral rehabilitation after treatment for head and neck cancer. The animals were irradiated with increasing irradiation doses to detect gradations in irradiation damage that could eventually lead to the development of ORN. Imaging was done at baseline with conventional CT- and MR-imaging followed by irradiation (described in ${ }^{14}$ ). 3 months later surgery was performed. At 6 months after radiation DE-CT and MR-imaging were performed. All steps were performed under general anesthesia.

For this study, 15 healthy, adult, 18-month-old female Göttingen minipigs were used (weight 35-45 kilogram). 4 animals each were assigned to 1 of the 3 groups receiving an equivalent dose (EQD) of 0, 25, $50 \mathrm{~Gy}$, and 3 animals received 70 Gy (irradiation was given in 2 fractions of $0,6.5,9.7$, and $11.8 \mathrm{~Gy}$ ). Irradiation was performed with a 6 megavolt linear accelerator (Linac Siemens, Erlangen, Germany). The linear quadratic model was used to calculate the equivalent dose, and an alpha/beta ratio of 3 was used for late responding tissues. ${ }^{15}$

3 months after irradiation, surgery of the mandible involved extraction of all premolars and molars on the left side only. In the same session, 4 submerged dental implants were placed in the left mandible.

\section{Imaging acquisition}

Post-irradiation imaging was performed using dual-source CT (Siemens Somatom Definition Flash, Siemens Erlangen, Germany. Parameters: tube A Sn140kVp, 225 mAs; tube B 80kVp - $450 \mathrm{mAs}, \mathrm{CDTI}_{\mathrm{vol}} 38.15 \mathrm{mGy}$, slice thickness $0.6 \mathrm{~mm}$, FOV $300 \mathrm{~mm}$, and Matrix 512x512). The STIR sequence was used for the detection of bone marrow edema of the mandible using a head-coil (1.5 Tesla Intera, Phillips Healthcare, Best, the Netherlands; STIR sequence: TR/TE=3900/15 ms, FOV $240 \mathrm{~mm}$ and slice thickness 3.0 $\mathrm{mm})$.

\section{Data analyses}

A 3-material decomposition algorithm (Syngo multimodality workspace, version VE40A, Siemens Erlangen, Germany) was used to calculate the VNCa images by preselecting the Liver virtual non-contrast setting with the $r$ set at 1.71 as described by Guggenberger et al. ${ }^{12}$ The images then were sent to the PACS system. 
Two observers (AP, LP) placed 4 ROI's in the VNCa images at the marrow space and 4 ROI's on the buccal cortex on both sides to measure the HU values.

The agreement of the HU measurements of both observers was investigated. For the designated areas, the mean value was calculated from the individual values of both observers per side and of both the marrow and cortex.

The STIR images were scored by two observers (AP, AS) for bone marrow edema on a scale of 0 to 3 (Table 1). The scores were compared to the mean VNCa HU value measurements. The MR images and the VNCa images of the left and right sides were analyzed together and also separately to investigate the effect of the intervention on the left side.

Table 1. The STIR scores of bone marrow signal intensity in the mandible.

$0=$ no abnormal increase in marrow signal intensity

$1=$ minor increase in marrow signal intensity

$2=$ moderate increase in marrow signal intensity

3 = major increase in marrow signal intensity

The data were transferred to an electronic sheet (Excel ${ }^{\mathrm{TM}}$, Microsoft Office 2010, Redmond, WA, USA) and then calculated using SPSS 20 ${ }^{\mathrm{TM}}$ (Statistical Package for the Social Sciences, SPSS Inc., Chicago, IL, USA).

To evaluate the agreement of $\mathrm{HU}$ measurements between the two observers, the intraclass correlation coefficient was calculated. The Independent Student's T test was used to compare the $\mathrm{HU}$ values of the marrow and the cortex on the left and the right sides. For comparison of HU values to the STIR scores, ANOVA was used and checked with the Hochberg test for significance. This was done for the left and right side together and separately. For the left and right side together, an $\mathrm{R}^{2}$ linear coefficient was calculated. The P-value was considered significant for $p<0.05$.

\section{RESULTS}

The MR-based marrow edema scores of the dose groups are shown in figure 1. The STIR images showed increased signal intensity in the higher radiation groups (figure 2).

The HU value measurements of both observers had an intra-class correlation coefficient of 0.896 for marrow measurements of the left and 0.903 for the right mandibles. The $\mathrm{HU}$ value measurements of the cortex of both observers showed an intra-class correlation coefficient of 0.367 for the left and 0.419 for the right mandibles (Table 2 ). 


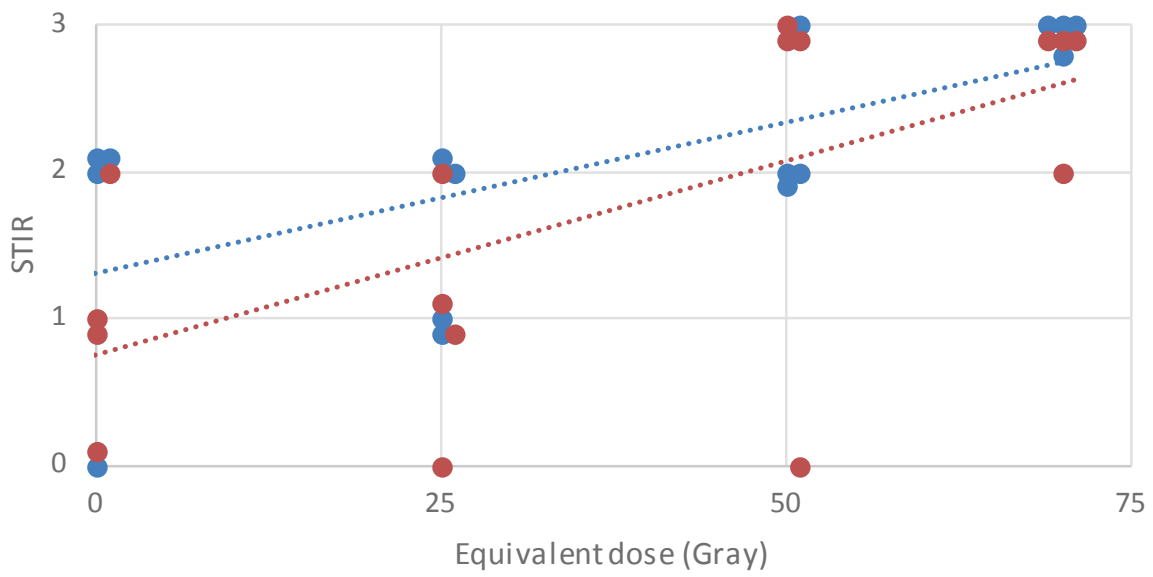

- left $\quad$ right $\ldots . . .$. . Linear (left) $\ldots . . .$. Linear (right)

Figure 1. MR imaging STIR-weighted sequences scores of the left and right mandibles 6 months after irradiation plotted against equivalent dose group (EQD). The number of dots given corresponds to the number of animals.

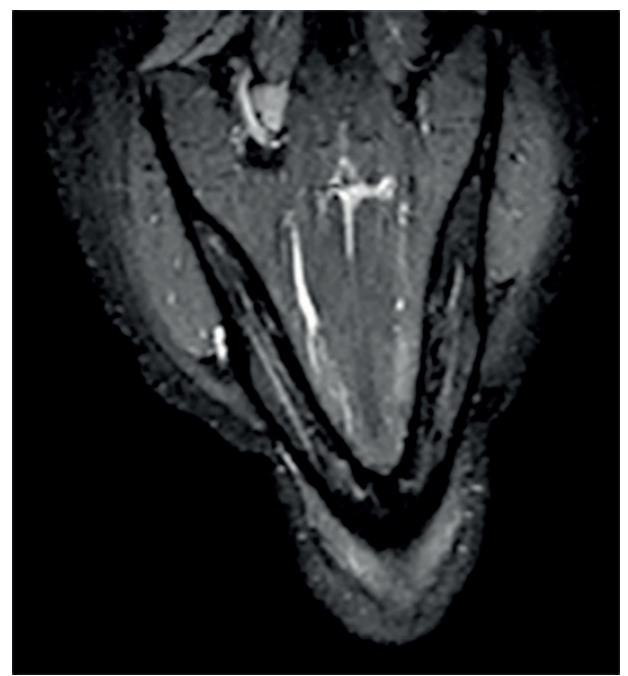

a.

Figure $2 \mathrm{a}$ and $\mathrm{b}$. Axial-orientated STIR images parallel to the corpus of the mandible. a: bilateral view of a normal mandible with a centrally located normal inferior alveolar nerve bundle, EQD OGy, no increased signal intensity is present. b: bilateral view of the mandible irradiated with EQD 70 Gy, demonstrating a major increase in signal intensity of the bone marrow, consistent with a bone marrow edema pattern.

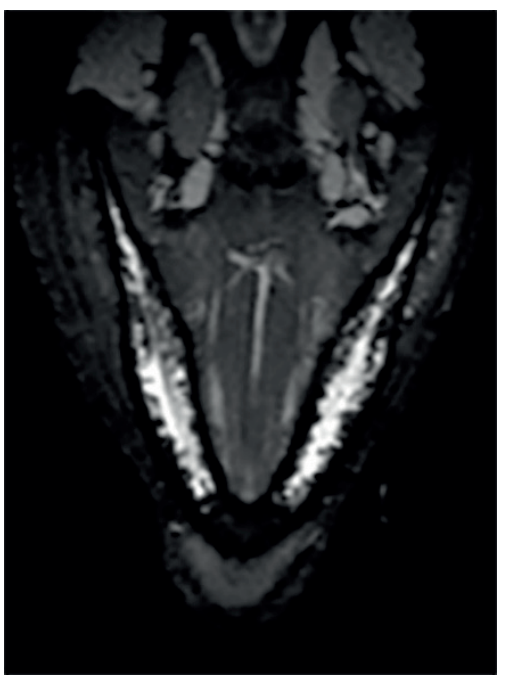

b. 
Table 2. The Intra-class Correlation Coefficient of the $\mathrm{HU}$ value measurements between the two observers

\begin{tabular}{llll}
\hline & Single measures & Significance & Average measures \\
\hline Cortex left & 0.225 & 0.038 & 0.367 \\
Marrow left & 0.812 & 0.000 & 0.896 \\
Cortex right & 0.265 & 0.020 & 0.419 \\
Marrow right & 0.824 & 0.000 & 0.903 \\
\hline
\end{tabular}

The mean HU values per STIR score for the left and right mandibles were calculated and shown in table 3.

Table 3. The mean HU values measured in the cortex and marrow on the left and the right sides. The mean $\mathrm{HU}$ values per STIR score and total are given (marrow: total left - right; $p>0.05$, cortex: total left - right; $p>0.05$ ).

\begin{tabular}{|c|c|c|c|c|c|c|}
\hline \multirow[b]{2}{*}{ STIR } & \multicolumn{3}{|c|}{ Cortex Left } & \multicolumn{3}{|c|}{ Marrow left } \\
\hline & $\mathrm{N}$ & Mean HU value & Standard Deviation & $\mathrm{N}$ & Mean HU value & Standard Deviation \\
\hline 0 & 1 & 64.51 & & 1 & -96.49 & \\
\hline 1 & 2 & 55.10 & 11.16 & 2 & -74.78 & 62.64 \\
\hline 2 & 8 & 60.35 & 2.95 & 8 & $-82.56\} *$ & 16.76 \\
\hline 3 & 4 & 52.39 & 21.82 & 4 & -23.05 & 29.05 \\
\hline \multirow[t]{2}{*}{ Total } & 15 & 57.80 & 11.47 & 15 & -66.58 & 36.91 \\
\hline & \multicolumn{3}{|c|}{ Cortex Right } & \multicolumn{3}{|c|}{ Marrow Right } \\
\hline STIR & $\mathrm{N}$ & Mean HU value & Standard Deviation & $\mathrm{N}$ & Mean HU value & Standard Deviation \\
\hline 0 & 3 & 66.73 & 1.85 & 3 & -105.24 & 27.80 \\
\hline 1 & 4 & 64.03 & 1.68 & 4 & -98.16 & 20.50 \\
\hline 2 & 3 & 63.19 & 0.67 & 3 & -58.56 & 32.65 \\
\hline 3 & 5 & 61.65 & 8.95 & 5 & -44.00 & 43.40 \\
\hline Total & 15 & 63.61 & 5.25 & 15 & -73.60 & 40.49 \\
\hline
\end{tabular}

* = significant $\mathrm{p}=0.015$

The total mean HU value of the VNCa images was higher on the left (operated and irradiated) than the right (irradiated) side, however this difference was not significant (marrow: L -66.6; R -73.6; $p>0.05$ ) (cortex: $L$ 63.6; $R$ 57.8; $p>0.05$ ). An increase of signal intensity at STIR imaging was accompanied by an increase in the mean $\mathrm{HU}$ value of the VNCa images of the bone marrow (Table 3).

Comparing the bone marrow edema scores based on STIR to the mean HU values of the left and right sides, we found a significant difference between the groups (ANOVA $p=0.002)$. The Hochberg test was significant between 0 and $3(p=0.005)$, between 1 and $3(p=0.01)$, and between 2 and $3(p=0.031)$. (Table 4). The $R^{2}$ linear coefficient was 0.388 (Figure 3). 


\section{CHAPTER 6}

On the right side, the STIR scores were compared with the mean HU values and were not significant between the groups (ANOVA $p=0.072$ ). This was confirmed by the Hochberg test which also demonstrated non-significant values (Table 3).

Table 4. Mean HU values measured in the mandibular bone marrow. Mean HU values per STIR-score and total are given.

$\begin{array}{llll}\hline \multirow{2}{*}{\text { STIR }} & \multicolumn{2}{l}{\text { Mean HU values marrow left and right }} \\
$\cline { 2 - 4 } & $\left.\mathrm{N} & \text { Mean HU value } & \text { Standard Deviation } \\
\hline 0 & 4 & -103.06 \\
1 & 6 & -90.37 \\
2 & 11 & -76.01 \\
3 & 9 & -34.69\end{array}\right\} *$\begin{tabular}{l}
34.39 \\
23.14 \\
37.15 \\
Total \\
\hline
\end{tabular}

*=significant

$2-3 p=0.031$

$1-3 p=0.01$

$0-3 p=0.005$

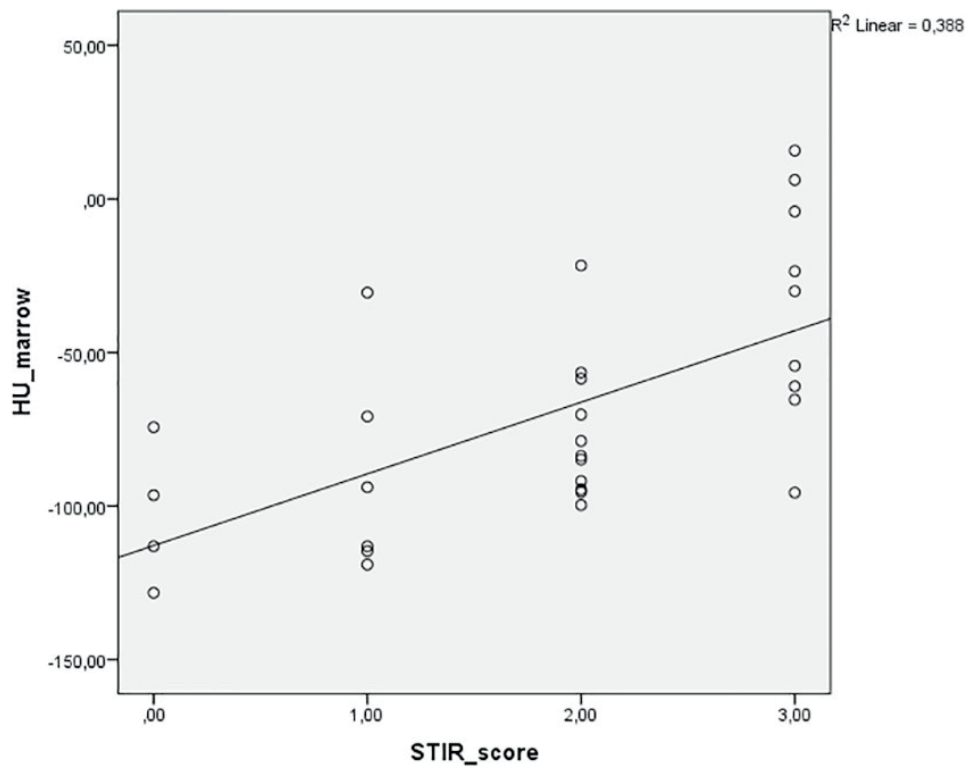

Figure 3. Scatterplot comparing the MR imaging STIR scores to the mean HU values of both sides of the mandibular bone marrow. $R^{2}=0.388$

On the intervention side, the STIR scores were compared with the mean HU values and this demonstrated a significant difference between the groups (ANOVA $p=0.015$ ). The Hochberg test was significant between 2 and $3(p=0.015)$ and non-significant between 1 
and 2 and between 1 and 3 . For the score 0 , only one mean was available, so this was not possible to test (Table 3).

\section{DISCUSSION}

CT and MR-imaging findings in ORN are complimentary. CT is better for cortical disruption and trabecular changes, whereas MR is superior in detection of increased water content as is bone marrow edema., 4,16

If $\mathrm{CT}$ is able to detect bone marrow edema next to cortical destruction it could, in theory, allow for a one stop shop for the detection of irradiation effects and ORN.

We found in our ORN animal model that DE-CT was able to detect edema of the mandible as compared to MR imaging. The mean HU values on the VNCa images were significantly correlated with the STIR-scores, which were used as the standard for the degree of bone marrow edema. The density measurements of the two investigators matched at that of the marrow of the mandible. However, at the cortex the measurements of the two investigators did not match. This could be due to the smaller $\mathrm{ROI}$, the slightly different positioning of the ROI tool, or the dense architecture of the cortex with only a small non-mineralized tissue content. As to be expected by the dense architecture and the low signal intensity at the cortex on MR, we were not able to demonstrate edema in the mandibular cortex.

An interesting finding is that although the surgical procedure was performed on the left side, we did not find a significant difference between the overall mean HU values of both sides. This was confirmed by the STIR scores, which did not differ much between both sides either. It is likely that the edema was caused by the surgery itself had resolved in most animals. Pathophysiologically, we think that the edema found was caused only by a reaction of the bone to the irradiation, and that the reaction was prolonged bone regeneration. ${ }^{16}$

Our results are in line with the small amount of studies that investigated bone marrow edema patterns using VNCa images. Pache et al. compared VNCa HU values with MR imaging and found that DE-CT could assess bone marrow involvement in posttraumatic bone bruises of the knee. ${ }^{11}$ They advocate the application of color-coded images for easier interpretation of the attenuation changes in the bone marrow. ${ }^{11}$ We did not use color-coded images for this study because we choose to evaluate the quantified density as are $\mathrm{HU}$ values, but their use could in theory increase the accuracy of visual interpretation and easily interpretation in clinical practice (Figure 4). Good correlations between the $\mathrm{HU}$ values from the VNCa and the MR imaging concerning post-traumatic bone marrow edema in the ankle ${ }^{12}$ and vertebra ${ }^{10}$ were published. All of these studies used adapted Liver VNC post-processing with an adjusted $r$. Different values of $r$ were mentioned in the literature. We choose an $r$ of 1.71 in line with Guggenberger because 
the ankle was the closest in scanned volume to the mandible. ${ }^{12}$ A specific choice of $r$ can influence the exact measurements of the $\mathrm{HU}$ values, but the optimal $r$ value has not yet been identified.

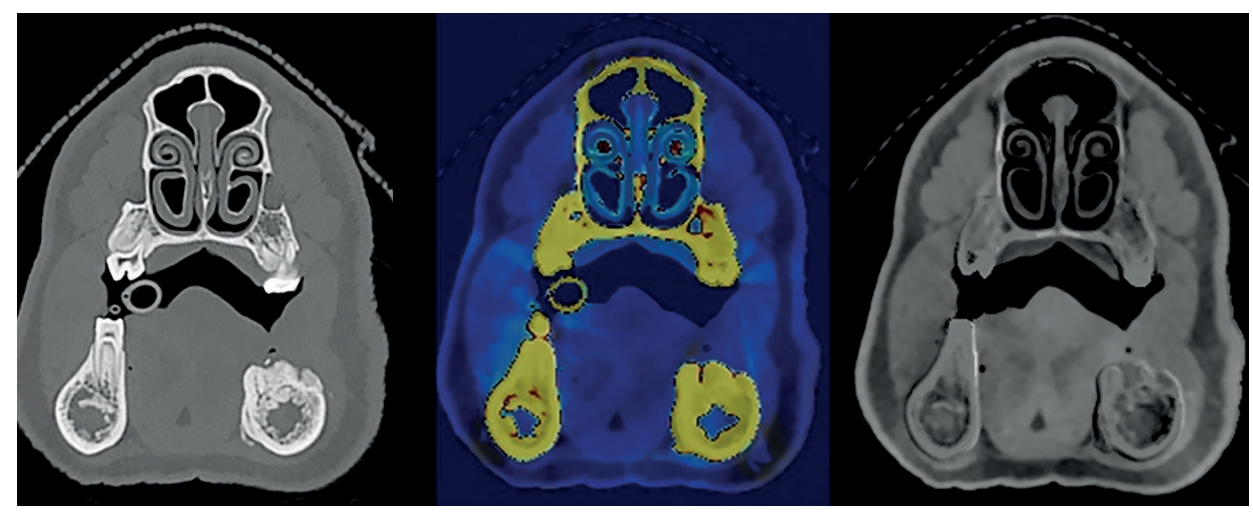

Figure 4. DE-CT images: mixed image (comparable with conventional $120 \mathrm{kV}_{\mathrm{p}} \mathrm{CT}$ image), fusion image, and VNCa image.

Other authors ${ }^{10,12,17}$ used T1-weighted and T2-weighted sequences for the detection of bone marrow edema on MR, whereas we choose STIR because it is ideally used for the detection of bone marrow edema. ${ }^{18}$

The studies mentioned above were based upon relatively homogenously composed bony structures originating from the epiphysis and the diaphysis. The mandible has a distinct, different embryological origin from vertebra, knee and ankle, and there is a clear difference between cortex and marrow. Despite this, we were still able to confirm the relationship between the $\mathrm{HU}$ value and the bone marrow edema.

In our ORN animal model, surgery with tooth extraction and implementation of implants was carried out as this is often the case in head and neck cancer patient groups.

The implants used can cause some scattering on CT imaging due to their almost pure titanium composition. because of the prone position of the animal in the gantry, the images of the mandible had a coronal orientation, the scattering only occurred locally at the mandible. We believe these artifacts did not influence the measurements, and the observers made sure not to measure anywhere near the implants or in the scatter artifacts. The signal intensities on STIR were not significantly influenced by the titanium content of the implants.

In case of metallic implants in the area of investigation, DE-CT can allow for metallic artifact reduction via mono-energetic reconstructions. ${ }^{5,19,20}$ At this time, the VNCa scoring would still be hindered by the presence of metal-induced streak artifacts, 
perhaps further improvements in the decomposition algorithms could decrease this interference.

The limitation of the study was the small number of animals. Despite the number being limited due to ethical considerations, we were able to demonstrate a correlation between the $\mathrm{HU}$ values of the VNCa images compared to the edema found with MR imaging.

Our results raise the possibility of single-modality imaging with DE-CT for the simultaneous detection of structural bone changes, as there are cortical disruptions and trabecular changes together with bone marrow edema. Before clinical implementation, this should be verified in a human population. Future research should aim at the clinical validation and implementation of DE-CT as a single modality in the detection and diagnosis of radiation induced changes and ORN.

In conclusion next to the ability of CT to depict cortical destruction and trabecular bone resorption, VNCa images derived from DE-CT are able to demonstrate bone marrow edema in radiation-induced bone changes in the mini-pigs' mandible. This opens up the possibility of one-stop-shop imaging of radiation induced changes and ORN of the mandible 


\section{REFERENCES}

1. Lyons A and Ghazali N, Osteoradionecrosis of the jaws: current understanding of its pathophysiology and treatment. Br J Oral Maxillofac Surg, 2008. 46(8): p. 653-60.

2. Teng MS and Futran ND, Osteoradionecrosis of the mandible. Curr Opin Otolaryngol Head Neck Surg, 2005. 13(4): p. 217-21.

3. Hermans R, Fossion E, loannides C, Van den Bogaert W, Ghekiere J and Baert AL, CT findings in osteoradionecrosis of the mandible. Skeletal Radiol, 1996. 25(1): p. 31-6.

4. Bachmann G, Rossler R, Klett R, Rau WS and Bauer R, The role of magnetic resonance imaging and scintigraphy in the diagnosis of pathologic changes of the mandible after radiation therapy. Int J Oral Maxillofac Surg, 1996. 25(3): p. 189-95.

5. Postma AA, Das M, Stadler AA and Wildberger JE, Dual-Energy CT: What the Neuroradiologist Should Know. Curr Radiol Rep, 2015. 3(5): p. 16.

6. Johnson TR, Dual-energy CT: general principles. AJR Am J Roentgenol, 2012. 199(5 Suppl): p. S3-8.

7. Johnson TR, Krauss B, Sedlmair M, Grasruck M, Bruder H, Morhard D, Fink C, Weckbach S, Lenhard M, Schmidt B, Flohr T, Reiser MF, and Becker CR, Material differentiation by dual energy CT: initial experience. Eur Radiol, 2007. 17(6): p. 1510-7.

8. Mileto A, Marin D, Ramirez-Giraldo JC, Scribano E, Krauss B, Mazziotti S and Ascenti G, Accuracy of contrast-enhanced dual-energy MDCT for the assessment of iodine uptake in renal lesions. AJR Am J Roentgenol, 2014. 202(5): p. W466-74.

9. $\mathrm{Yu} \mathrm{L}$, Leng $\mathrm{S}$ and $\mathrm{McCollough} \mathrm{CH}$, Dual-energy CT-based monochromatic imaging. AJR Am J Roentgenol, 2012. 199(5 Suppl): p. S9-S15.

10. Wang CK, Tsai JM, Chuang MT, Wang MT, Huang KY and Lin RM, Bone marrow edema in vertebral compression fractures: detection with dual-energy CT. Radiology, 2013. 269(2): p. 525-33.

11. Pache G, Krauss B, Strohm P, Saueressig U, Blanke P, Bulla S, Schafer O, Helwig P, Kotter E, Langer M, and Baumann T, Dual-energy CT virtual noncalcium technique: detecting posttraumatic bone marrow lesions--feasibility study. Radiology, 2010. 256(2): p. 617-24.

12. Guggenberger R, Gnannt R, Hodler J, Krauss B, Wanner GA, Csuka E, Payne B, Frauenfelder T, Andreisek $\mathrm{G}$ and Alkadhi $\mathrm{H}$, Diagnostic performance of dual-energy CT for the detection of traumatic bone marrow lesions in the ankle: comparison with MR imaging. Radiology, 2012. 264(1): p. 164-73.

13. Thomas C, Schabel C, Krauss B, Weisel K, Bongers M, Claussen CD and Horger M, Dual-energy CT: virtual calcium subtraction for assessment of bone marrow involvement of the spine in multiple myeloma. AJR Am J Roentgenol, 2015. 204(3): p. W324-31.

14. Poort $\amalg$ BG, Böckmann RA, Hoebers FJ, Houben R, Postma AA, Kessler PAWH Is there a change in bone mineral density changes of the mandible in Göttingen

Minipigs after irradiation in correlation with radiation dose and implant surgery? J Oral Maxillofac Surg, 2014. 72(11): p. 2149-2156.

15. Fowler JF, Tome WA, Fenwick JD and Mehta MP, A challenge to traditional radiation oncology. Int J Radiat Oncol Biol Phys, 2004. 60(4): p. 1241-56.

16. Store G, Smith HJ and Larheim TA, Dynamic MR imaging of mandibular osteoradionecrosis. Acta Radiol, 2000. 41(1): p. 31-7.

17. Pache G, Bulla S, Baumann T, Bayer J, Reising K, Strohm P, Langer M and Blanke P, Dose reduction does not affect detection of bone marrow lesions with dual-energy CT virtual noncalcium technique. Acad Radiol, 2012. 19(12): p. 1539-45.

18. Vanhoenacker FM and Snoeckx A, Bone marrow edema in sports: general concepts. Eur J Radiol, 2007. 62(1): p. 6-15.

19. Takrouri HS, Alnassar MM, Amirabadi A, Babyn PS, Moineddin R, Padfield NL, BenDavid G and Doria AS, Metal Artifact Reduction: Added Value of Rapid-Kilovoltage-Switching Dual-Energy CT in Relation to Single-Energy CT in a Piglet Animal Model. AJR Am J Roentgenol, 2015. 205(3): p. W352-9. 
20. Srinivasan A, Hoeffner E, Ibrahim M, Shah GV, LaMarca F and Mukherji SK, Utility of dual-energy CT virtual keV monochromatic series for the assessment of spinal transpedicular hardware-bone interface. AJR Am J Roentgenol, 2013. 201(4): p. 878-83. 



\section{CHAPTER 7}

The histological and histomorphometric changes in the mandible after radiotherapy. An animal model.

L.J. Poort, J.H.B. Ludlage, N. Lie, R.A. Böckmann, J.C.E. Odekerken, F.J. Hoebers, P.A.W.H. Kessler

Submitted to Radiation Research Journal 


\section{ABSTRACT}

Background and purpose Approximately $5 \%$ of irradiated head and neck cancer patients develop osteoradionecrosis of the mandible. The current non-surgical treatment options for osteoradionecrosis have limited effects and are based on a small number of studies. Therefore, we aimed to enhance the understanding of the pathophysiology of osteoradionecrosis by investigating changes induced by external irradiation in mini-pigs.

Materials and methods Sixteen Göttingen minipigs were divided into 4 groups for the application of equivalent radiation dosages of 0 Gy, 25 Gy, 50 Gy and 70 Gy. Thirteen weeks after irradiation, the left lateral teeth the mandible were removed and implants were placed. The pigs were sacrificed 26 weeks after irradiation, and the bone samples were stained with Masson's trichrome.

Results The amount of fibrosis, resorption lacunae, necrosis, and the ratio of woven/lamellar bone were increased after higher radiation dosages and surgery. The inferior alveolar artery's diameter was reduced depending on the irradiation dosages. In general, the rate of bone remodeling decreased after irradiation.

Conclusion Both surgery and increasing irradiation dosages cause architectural bone changes and damage the vascularization. This might result in a chronic hypoxic state of the mandibular bone. In general, the bone formation rate was markedly decreased after radiotherapy. 


\section{INTRODUCTION}

Osteoradionecrosis (ORN) of the mandible is a severe complication after radiotherapy (RT) and develops in approximately $2.6 \%-15 \%$ of head and neck cancer patients. ${ }^{1}$ The most accepted definition for ORN is: 'irradiated bone becomes devitalized and is exposed through the overlying skin or mucosa, without tumor recurrence, and does not heal within 3 months'. ${ }^{2}$ The current non-surgical treatment options for ORN, like hyperbaric oxygen therapy and medical treatment, are based on controversial theories and on a limited number of studies. ${ }^{3,4}$

The most accepted theory on the pathophysiology is the development of a combination of hypo-cellularity and hypo-vascularity in a hypoxic environment. ${ }^{5}$ Radiation obviously damages the vascular system, which causes a decreased oxygen supply to the irradiated tissues, in this case, the head and neck region. Time and local conditions seem to play an important role in the development and risk assessment of ORN. ${ }^{5,6}$

Recently, Delanian et al. proposed another theory based on RT-induced fibro-atrophic tissue changes. They concluded that osteoclastic giant cells are damaged more by irradiation than the vascular system. This process is associated with increased levels of myofibroblasts and low levels of proliferative fibroblasts. The deregulation and activation of the fibro-osseous activity will result in a constant fibrotic reorganization of the tissues. The final path resembles the theory described by Marx et al., resulting in a hypoxic environment with the risk of complete tissue breakdown. ${ }^{7}$

Some models for the development of ORN on cellular level found that ORN hypoxia induces profound deregulation of angiogenic, chemokine, and inflammatory factors. ${ }^{8}$ The limited number of histological studies shows discrepancies with regard to the results and justifies the development of a large-animal model to translationally demonstrate the clinical and histomorphological effects of RT in varying doses in the head and neck on the development of ORN.

The goal of this study was to establish an appropriate and efficient mini-pig model to study ORN in the head and neck. We hypothesized that an increasing irradiation dose will result in a progressive decrease in bone remodeling activity and vascularization and increasing levels of fibrosis, leading to clinical and morphological signs of ORN.

\section{METHODS}

The experiment was conducted in accordance with the European Community Guidelines for the Protection of (Laboratory) Animals. This study was part of a larger experimental animal study investigating irradiation effects on the mandible. ${ }^{9}$ The local animal ethical committee approved the study (DEC 2011-127). 


\section{Study design}

Sixteen healthy adult female Göttingen minipigs were used, aged 18 months at start of experiment and with a mean weight of $40 \mathrm{~kg}$.

\section{Study Variables}

Four pigs were randomly divided into one of the 4 research groups stratified by the respective radiation dose received, comprising the control, $25 \mathrm{~Gy}, 50 \mathrm{~Gy}$ or $70 \mathrm{~Gy}$ irradiated groups. The primary outcomes were the bone vitality, bone formation rate and vascularization. The two study variables were radiation dose and surgery. No other variables were related to the outcome of this study.

\section{Procedures}

A 6-megavolt linear accelerator (Linac Siemens, Erlangen, Germany) with two opposing lateral beams was used to irradiate the entire mandible. Two fractions with dosages of 6.5 Gy, 9.7 Gy and $11.8 \mathrm{~Gy}$, equivalent to $25 \mathrm{~Gy}, 50 \mathrm{~Gy}$ or $70 \mathrm{~Gy}$, were used with a time interval of 48 hours. This biological effective dose was calculated with a linear quadratic model with the assumption of $\alpha / \beta$ ratio of $3 .^{10}$

Thirteen weeks after irradiation, teeth were extracted and dental implants were placed under general anesthesia to simulate a typical surgical trauma in the jaws. All molars and premolars on the left side of the mandible were extracted, and subsequently four Biocomp dental implants (diameter $4 \mathrm{~mm}$ and length $12 \mathrm{~mm}$ ) were placed in each pig (Bio-Comp Industries BV, Vught, The Netherlands). Postoperatively, intramuscular pain medication (buprenorphine, Reckitt Benckiser Healthcare Hull, United Kingdom) and intramuscular antibiotics (amoxicillin, AST beheer BV Oudewater, the Netherlands) were administered for three days. The fluorochrome markers calcein green, xylenol orange and calcein blue were subcutaneously administered 2, 14 and 24 weeks after radiotherapy, respectively (Fluka, Sigma-Aldrich, Seelze, Germany).

After sacrifice, at 26 weeks after irradiation, the bone samples were fixated in $4 \%$ formaldehyde and subsequently embedded in polymethyl methacrylate (PMMA; Technovit 9100, Heraeus Kulzer, Hanau, Germany) and sectioned at a thickness of 78 $\mu \mathrm{m}$ with a saw microtome (Leica SP1600, Wetzlar, Germany). This was done to specifically analyze both implant and the surrounding bone. The samples were stained with Masson's trichrome using a Masson-Goldners trichrome staining kit (Carl Roth, Karlsruhe, Germany). Four samples per side were taken and were subsequently analyzed under a light microscope (Carl Zeiss AG 1600, Oberkochen, Germany) and pictures were made on five different locations of the cross-sections with magnifications of $25 x$ and 50x. These five locations were evenly distributed over the entire circumference of the mandibular bone. 
The specimens were analyzed for bone vitality and vascularization. The bone vitality was measured by evaluating the amount/size of resorption lacunae, the ratio of woven/lamellar bone and the levels of necrosis and fibrosis. This was assessed with a semi-quantitative scoring method and expressed as not present $(-)$, mild $(+)$, moderate $(++)$ and severe $(+++)$. The samples were analyzed by four independent observers (LP, $\mathrm{HL}, \mathrm{NL}$, and RB), blinded for individual treatment given with 1-50x magnification. Based on these results, the final scores were determined by two observers. This scoring method was based on the scoring method of Fenner et al and Aitasalo et al. ${ }^{11,12}$

The inferior alveolar artery (IAA) provides the main blood supply for the mandibular body. The IAA was measured according to the following parameters: thickness of the tunica intima, intima-media thickness and the diameter of the vascular lumen at a magnification of 50x. The above-mentioned parameters were measured with Image J software (Image processing and analysis in Java, NIH, Bethesda, Maryland, USA).

The bone formation rate was determined by measuring the distance between fluorochrome tracings in the exocortical bone of the lateral mandibular cortex. At five different locations, the images were analyzed under a fluorescence microscope with a magnification of 100x (Leica Microsystems, Leitz DMRB, Wetzlar, Germany). For every location, three images were taken at the exact same location with different excitation wavelengths. Photoshop (Adobe Photoshop CS6, Adobe Systems Software, San Jose, CA, USA) was used to process these images, and the distances between the fluorochrome tracings were measured with the Image J program. Period 1 was defined as the distance between calcein green and xylenol (marking week 2 and 14 after irradiation), and period 2 was defined as the distance between xylenol orange and calcein blue (marking week 14 and 24 after irradiation).

\section{Statistical analysis}

The data were incrementally entered into electronic sheets (Excel, Microsoft Office 2010, Microsoft, Redmond, WA, USA) during the study period and processed with IBM SPSS version 22 (IBM, Somers, New York, USA). The differences between the groups were measured with the Mann-Whitney $U$ test. Because of the small study population and corresponding small power, we used non-parametric tests, as a normal distribution could not be assumed.

\section{RESULTS}

Two animals were diagnosed with clinical ORN with exposed bone centrally in the irradiated area, one pig from the $50 \mathrm{~Gy}$ group and one from the $70 \mathrm{~Gy}$ group. The ORN animals displaying clinical ORN were histologically compared to the other animals of 
their groups. No differences could be found regarding the diameter of the vascular lumen, media intima thickness, and bone formation rate during period 1.

However, significant differences were found with regard to the tunica intima thickness and bone formation rate during period 2. The tunica intima thickness was increased in the clinical ORN pigs, and the bone formation rate during period 2 was decreased.

In general, the diameter of the vascular lumen decreased after irradiation in a dosedependent manner on both sides of the mandible, the operated and non-operated side. There were no significant differences (see Figure $1 \mathrm{~A}$ ). Figure $1 \mathrm{~A}-\mathrm{C}$ depicts the results of varying radiation doses on the vascular structure of the IAA. The operated and nonoperated sides of the mandible are compared with each other.

The bone vitality was assessed using a semi-quantitative method of measurement, and the values are listed in Table 1. In our study, the level of fibrosis, the number and size of resorption lacunae and the ratio of woven/lamellar bone increased in a manner dependent on radiation dosages and surgery. On the surgical side, there was an increased level of woven bone present and signs of necrosis. Table 1 describes the results in detail. Supplementary images depicts images of IAA, mandibular bone architecture and lamellar to woven bone increase.

Bone remodeling was markedly decreased after irradiation during both periods on the operated and non-operated sides (see Figure 2). During period 1, there were no significant differences in bone remodeling rates in between the varying irradiation groups. During period 2, however, the bone remodeling rates were significantly decreased with increasing radiation doses on the non-operated side. The bone remodeling rates of all groups were significantly increased during period 2 compared to period 1. Figure 3 depicts composed fluorochrome images.

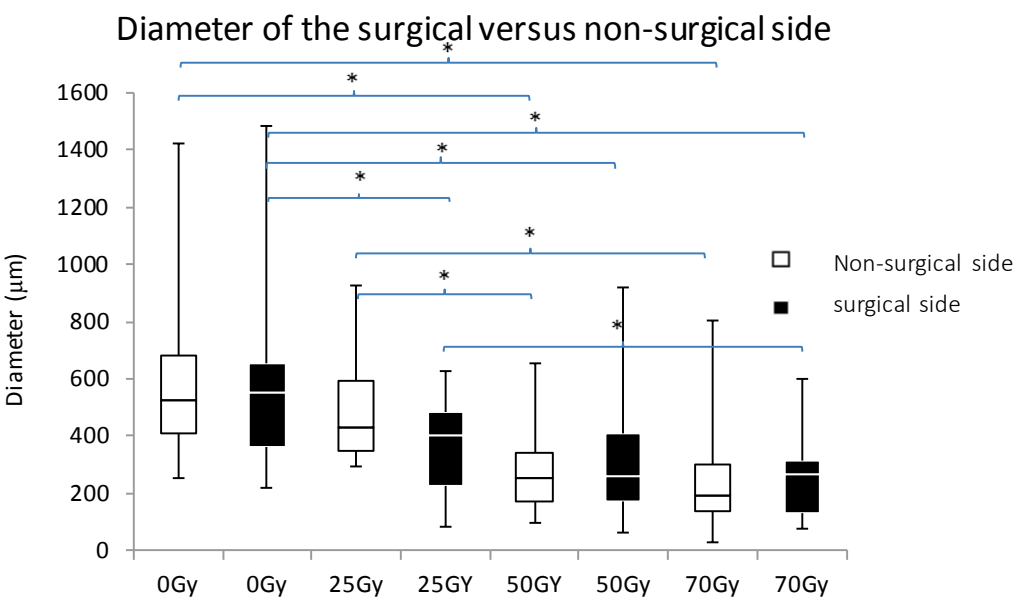

a. 
Tunica Intima thickness of the surgical versus non-

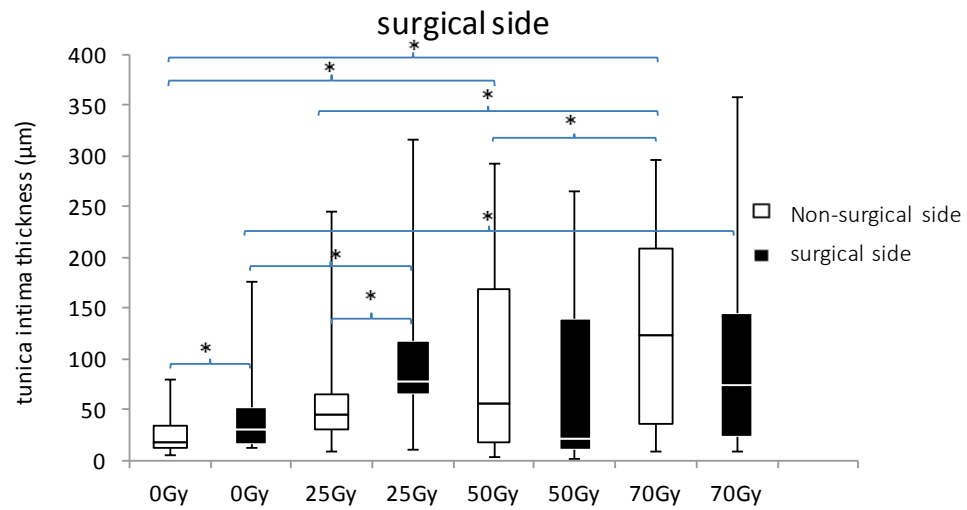

b.

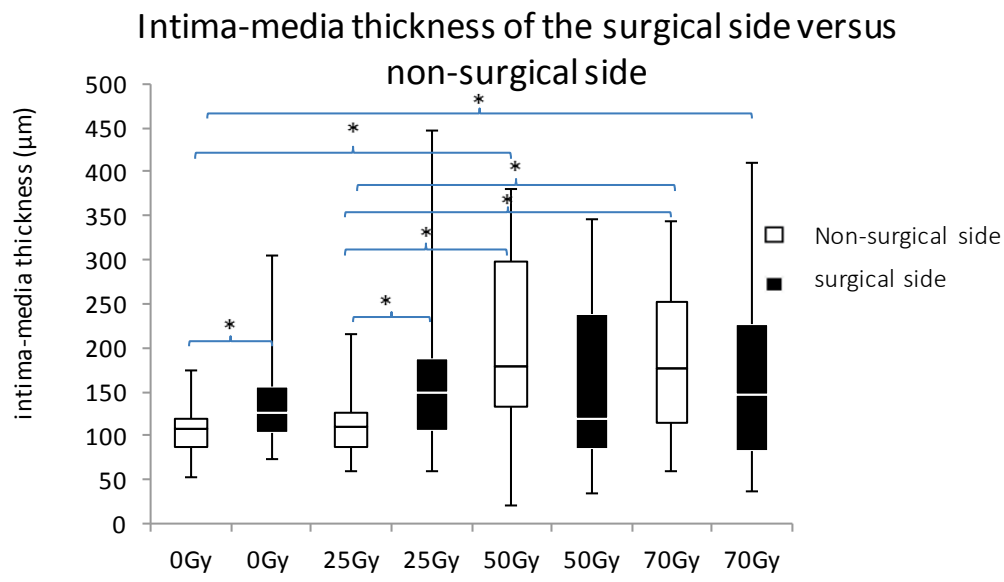

c.

Figure 1. Vascular changes of the in IAA depending on radiation doses and surgical trauma. Figure 1A: Effects on the diameter of the vascular lumen. Figure 1B: Effects on the thickness of the tunica intima Figure 1C: Effects on the intima-media thickness. Significant differences are marked with an asterisk * 


\section{CHAPTER 7}

Table 1. Results of semi-quantitative histological scoring. The amount of fibrosis, necrosis, lacunae forming and the ratio woven/lamellar bone dependent on radiation dose. " 0 " = not present, "+" =mildly present, " ++" $=$ moderately present and "+++" = highly present.

\begin{tabular}{|c|c|c|c|c|c|}
\hline & Fibrosis & Necrosis & Lacunae & Woven bone & Lamellar bone \\
\hline \multicolumn{6}{|l|}{ Control group } \\
\hline Non-surgical side & 0 & 0 & + & + & +++ \\
\hline Surgical side & 0 & 0 & + & ++ & +++ \\
\hline \multicolumn{6}{|l|}{ 25Gy group } \\
\hline Non-surgical side & 0 & 0 & + & + & +++ \\
\hline Surgical side & + & + & + & ++ & ++ \\
\hline \multicolumn{6}{|l|}{ 50Gy group } \\
\hline Non-surgical side & + & 0 & ++ & + & +++ \\
\hline Surgical side & + & + & ++ & ++ & ++ \\
\hline \multicolumn{6}{|l|}{ 70Gy group } \\
\hline Non-surgical side & + & 0 & ++ & ++ & +++ \\
\hline Surgical side & ++ & + & +++ & ++ & ++ \\
\hline
\end{tabular}

\section{Bone remodeling rate during period 1}
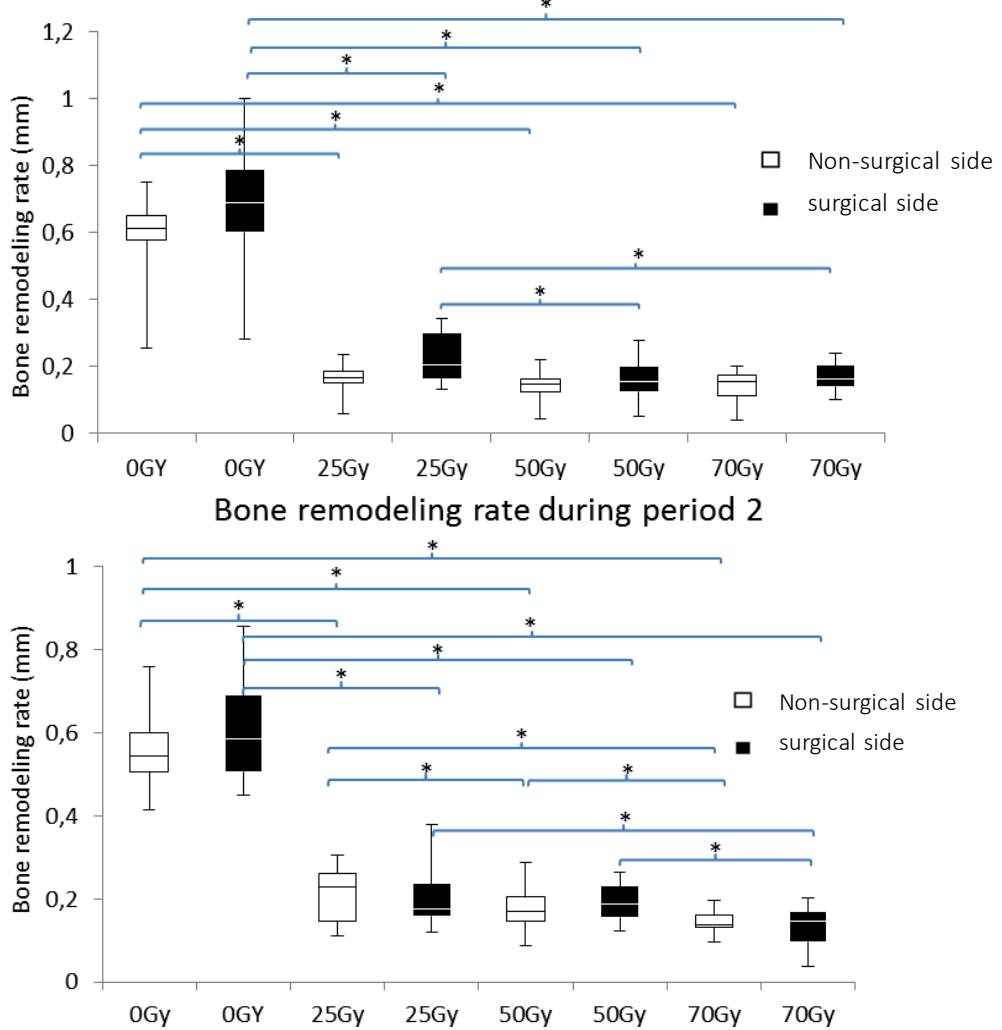

Figure 2. The bone formation rates over time during period 1 and period 2 dependent on radiation doses. 


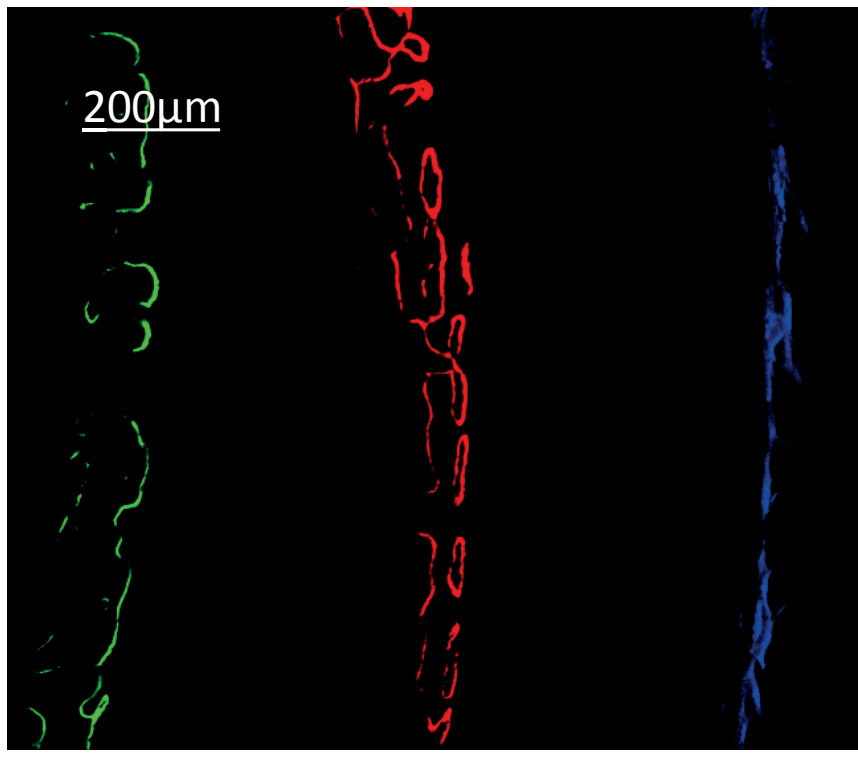

a.

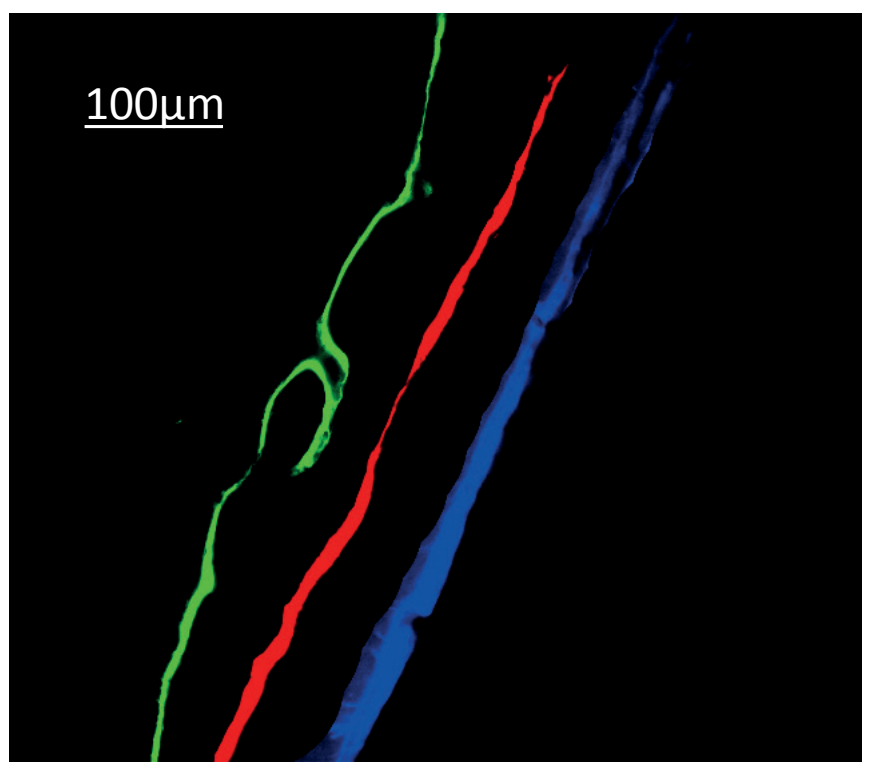

b.

Figure 3. Fluorescence microscopy images composed of an overlay image the fluorescence signaling in cortical bone, markers shown are calcein green, xylenol orange, calcein blue respectively. A 0Gy and B 70Gy animal. 


\section{DISCUSSION}

The IAA was studied intensively in this experiment because it is the main artery of the mandible. ${ }^{13}$ The diameter of the lumen was decreased after irradiation on the operated and non-operated sides. A decreased diameter could indicate a decreased blood flow to the tissue, and the nutrient supply could therefore be reduced. This decline in blood supply could be the main cause for the reduction of the healing capacity in the mandible. On the other side, the risk of vulnerability of irradiated tissues might be enhanced. ${ }^{14}$ In a study on the effects of RT on the aorta, Gillette et al. found that the diameter of the lumen was not decreased after irradiation. ${ }^{15}$ The difference in outcomes was probably caused by histological variations and the differences in the location and size in arteries studied. Larger arteries, such as the aorta, suffer less from the effects of irradiation compared to smaller arteries. ${ }^{16}$

The vascular changes of the artery were measured by the thickness of the tunica intima and the media-intima thickness. We found a dramatically thickened tunica intima after mandibular irradiation, with a wide spread in the effects of irradiation on the vascular changes. The individual variety of radiation effects could help to explain the unpredictability of the risk of ORN in humans. This variation in incidence was also found in our study, where one pig in the 50 Gy group and one in the 70 Gy group developed clinical ORN. Both of these pigs had an outspoken thickened tunica intima.

These findings are in line with the findings of Bras et al., where even in the larger arteries, such as the aorta, a thickening of the tunica intima was found. ${ }^{13,17}$ The thickening of the tunica intima is associated with increased fibrotic tissue formation, lipid deposition, and a subclinical change, which predicts the risk for vascular events. ${ }^{18}$ Surgical trauma did not have any significant influence on vascular changes. Only in the 25 Gy group did the tunica intima of the operated animals show an obvious increase in thickness compared to the control group.

Under normal conditions, the IAA is the main artery to the entire mandible. If the artery is blocked, angiogenesis or arteriogenesis can only partially restore the vascularization of the mandible. The angiogenesis is induced by hypoxia and inflammation. ${ }^{19}$ The facial artery and other collateral vessels will substitute the perfusion of the periosteum, mainly in the lateral aspects of the mandible and the symphyseal region. ${ }^{20,21}$ However, whether this revascularization of the mandibular body can restore the vascularization completely is unknown. The irradiation of the mandible causes vascular compromise, which might lead to ORN this also applies to the minipigs. ${ }^{22}$

To the best of our knowledge, this is the first study to investigate the effects of different irradiation dosages on bone vitality in a large animal model. Twenty-six weeks after radiotherapy, we found an increasing amount of fibrosis, resorption lacunae and an increased ratio of woven/lamellar bone that was dependent on irradiation dosages and surgery. The levels of necrosis were only increased after surgery in combination with irradiation. We only found microscopic changes indicating necrosis in irradiated and 
operated mandibular sides. In our study, we found that RT has effects on all tissues in the irradiated field.

Fibrotic changes were also seen in the surrounding tissues, such as the bone marrow, and in the operated region. Similar results were found in other studies, which found increasing levels of TGF- $\beta$ after irradiation and an association of TGF- $\beta$ with the development of fibrotic tissue. ${ }^{23,24}$ In normal wound healing, the fibrotic phenotype is a temporary phenotype and disappears after wound healing. However, after radiotherapy, the myofibroblasts remain excessively persistent in the irradiated area, resulting in a chronic fibrotic state of the tissue according to Delanian et al. ${ }^{7}$

The ratio of woven/lamellar bone was shifted towards a more woven bone-like phenotype after irradiation. Increased levels of woven bone indicate compromised bone, as woven bone is mechanically weaker. ${ }^{25}$ The levels of necrosis were increased after radiotherapy in combination with surgery. Necrosis normally predominates in the later stages of tissue after radiotherapy. ${ }^{7}$

The bone remodeling rates of the exocortical bone were remarkably decreased after irradiation. In the literature, contradicting results were found about the influence of radiotherapy on the bone remodeling rate. ${ }^{26,27}$ Three major cell types are involved in this process: osteoclasts, osteoblasts and osteocytes. The coupling between those cells is essential for balanced bone remodeling. ${ }^{28}$ This coupling is disturbed after radiotherapy, as described by Alwood et al. and others. ${ }^{27}$ They hypothesize that TNF- $\alpha$ has a central role in osteoclastogenesis. In response to RT, there is an increase in the levels of TNF- $\alpha$, thereby triggering the inflammatory response and stimulating the further release of several cytokines. The levels of RANK-L and M-SCF are increased by TNF- $\alpha$, and they are both essential for the differentiation, activation and survival of osteoclasts. $^{8,27}$ In the physiological situation, the effects of RANK-L are partially blocked by OPG, but after radiotherapy, this balance is disturbed by increased levels of RANK$\mathrm{L}^{27}$ This results in increased numbers and increased activation of osteoclasts, which will result in lower bone formation rates and increased bone resorption rates. ${ }^{27}$ This was also found in our study. The bone formation rate of ORN pigs was also reduced, indicating that $O R N$ reduces the bone formation rate.

Based on the results of fluorochrome staining, we found that the bone formation rates decreased after irradiation. The results of other animal studies in literature contradict these findings. ${ }^{8,26}$ The differences found might be based on the type of experimental animal used. We are convinced that the results related to bone regeneration provided by large-animal experiments with Göttingen mini-pigs will deliver more representative results, as the bone biology of the Göttingen mini-pig resembles that of humans much better than that of small-animal research models. ${ }^{8,26}$ The bone formation rates in our study were increased only immediately after surgery and decreased again in the later stages. Similar results were found by Berglundh et al. and Brasseur et al., who found that minor surgery can increase bone formation. ${ }^{29,30}$ 


\section{CHAPTER 7}

As in all animal-based studies, we had to accept some shortcomings in our study. In our model, the irradiation was given in two large fractions only, whereas in humans, RT is normally given in daily small fractions or is even hyper-fractionated. However, we found pathophysiological reactions similar to those in humans. Therefore, our model seems to be appropriate for the examination of side effects of irradiation in the head and neck. The histological sections used in this study for histomorphometric analysis were relatively thick. Therefore, it was not possible to examine the specimens at a larger magnification, which made it impossible to evaluate cellular processes and changes. Nevertheless, the parameters measured were of high quality.

Because cytokines and their receptors seem to play an essential role in the pathophysiological processes leading to ORN, future studies should evaluate RT-induced effects on the cellular level. Despite the limited observation time, two animals were observed to have clinical ORN, albeit early ORN.

In summary, we found typical RT-related tissue changes in all irradiated animals. In terms of surgical interventions, trauma to the irradiated mandible resulted in a higher risk of developing ORN. We found changes of the arterial wall of the IAA in all animals. The degree of vascular change was dose-dependent. A radiation dose-dependent imbalance between bone repair and osteoclastic activity was also seen.

After irradiation of the mandible in Göttingen mini-pigs, we found vascular damage, an increase in fibrotic tissue and high osteoclastic activity without a clear dose-dependent threshold. We believe that the vascular changes induced by RT are key to development of ORN.

\section{ACKNOWLEDGEMENTS}

We kindly thank BioComp Industries BV, Vught, the Netherlands, and the BOOA foundation of the NVMKA for partial funding of this animal experiment. 


\section{REFERENCES}

1. Lambade PN, Lambade D and Goel M, Osteoradionecrosis of the mandible: a review. Oral Maxillofac Surg, 2013. 17(4): p. 243-9.

2. Lyons A and Ghazali N, Osteoradionecrosis of the jaws: current understanding of its pathophysiology and treatment. Br J Oral Maxillofac Surg, 2008. 46(8): p. 653-60.

3. Delanian S, Depondt J and Lefaix JL, Major healing of refractory mandible osteoradionecrosis after treatment combining pentoxifylline and tocopherol: a phase II trial. Head Neck, 2005. 27(2): p. 114-23.

4. Shaw RJ and Dhanda J, Hyperbaric oxygen in the management of late radiation injury to the head and neck. Part I: treatment. Br J Oral Maxillofac Surg, 2011. 49(1): p. 2-8.

5. Marx RE, Osteoradionecrosis: a new concept of its pathophysiology. J Oral Maxillofac Surg, 1983. 41(5): p. 283-8.

6. Marx RE and Tursun R, Suppurative osteomyelitis, bisphosphonate induced osteonecrosis, osteoradionecrosis: a blinded histopathologic comparison and its implications for the mechanism of each disease. Int J Oral Maxillofac Surg, 2012. 41(3): p. 283-9.

7. Delanian $\mathrm{S}$ and Lefaix $\mathrm{JL}$, The radiation-induced fibroatrophic process: therapeutic perspective via the antioxidant pathway. Radiother Oncol, 2004. 73(2): p. 119-31.

8. Kitaura H, Kimura K, Ishida M, Kohara H, Yoshimatsu M and Takano-Yamamoto T, Immunological reaction in TNF-alpha-mediated osteoclast formation and bone resorption in vitro and in vivo. Clin Dev Immunol, 2013. 2013: p. 181849.

9. Poort $L \mathrm{BG}$, Böckmann RA, Hoebers FJ, Houben R, Postma AA, Kessler PAWH Is there a change in bone mineral density changes of the mandible in Göttingen

Minipigs after irradiation in correlation with radiation dose and implant surgery? J Oral Maxillofac Surg, 2014. 72(11): p. 2149-2156.

10. Fowler JF, Tome WA, Fenwick JD and Mehta MP, A challenge to traditional radiation oncology. Int J Radiat Oncol Biol Phys, 2004. 60(4): p. 1241-56.

11. Fenner M, Park J, Schulz N, Amann K, Grabenbauer GG, Fahrig A, Karg J, Wiltfang J, Neukam FW and Nkenke $E$, Validation of histologic changes induced by external irradiation in mandibular bone. An experimental animal model. J Craniomaxillofac Surg, 2010. 38(1): p. 47-53.

12. Aitasalo K, Bone tissue response to irradiation and treatment model of mandibular irradiation injury. An experimental and clinical study. Acta Otolaryngol Suppl, 1986. 428: p. 1-54.

13. Bras J, de Jonge HK and van Merkesteyn JP, Osteoradionecrosis of the mandible: pathogenesis. Am J Otolaryngol, 1990. 11(4): p. 244-50.

14. White JJ, Ram SJ, Jones SA, Schwab SJ and Paulson WD, Influence of luminal diameters on flow surveillance of hemodialysis grafts: insights from a mathematical model. Clin J Am Soc Nephrol, 2006. 1(5): p. 972-8.

15. Gillette EL, Powers BE, McChesney SL, Park RD and Withrow SJ, Response of aorta and branch arteries to experimental intraoperative irradiation. Int J Radiat Oncol Biol Phys, 1989. 17(6): p. 1247-55.

16. Fajardo LF, Is the pathology of radiation injury different in small vs large blood vessels? Cardiovasc Radiat Med, 1999. 1(1): p. 108-10.

17. Stewart JR, Fajardo LF, Gillette SM and Constine LS, Radiation injury to the heart. Int J Radiat Oncol Biol Phys, 1995. 31(5): p. 1205-11.

18. Osika W, Dangardt F, Gronros J, Lundstam U, Myredal A, Johansson M, Volkmann R, Gustavsson T, Gan LM and Friberg $P$, Increasing peripheral artery intima thickness from childhood to seniority. Arterioscler Thromb Vasc Biol, 2007. 27(3): p. 671-6.

19. Carmeliet P and Jain RK, Molecular mechanisms and clinical applications of angiogenesis. Nature, 2011. 473(7347): p. 298-307.

20. Hellem S and Ostrup LT, Normal and retrograde blood supply to the body of the mandible in the dog. II. The role played by periosteo-medullary and symphyseal anastomoses. Int J Oral Surg, 1981. 10(1): p. 3142. 


\section{CHAPTER 7}

21. Store $\mathrm{G}$ and Granstrom G, Osteoradionecrosis of the mandible: a microradiographic study of cortical bone. Scand J Plast Reconstr Surg Hand Surg, 1999. 33(3): p. 307-14.

22. Saka B, Wree A, Anders $L$ and Gundlach KK, Experimental and comparative study of the blood supply to the mandibular cortex in Gottingen minipigs and in man. J Craniomaxillofac Surg, 2002. 30(4): p. 219-25.

23. Andarawewa KL, Paupert J, Pal A and Barcellos-Hoff MH, New rationales for using TGFbeta inhibitors in radiotherapy. Int J Radiat Biol, 2007. 83(11-12): p. 803-11.

24. Anscher MS, Kong FM, Murase T and Jirtle RL, Short communication: normal tissue injury after cancer therapy is a local response exacerbated by an endocrine effect of TGF beta. Br J Radiol, 1995. 68(807): p. $331-3$

25. Currey JD, The structure of Bone tissue., in Bones: Structure and Machanics. 2006, Princeton University Press: Princeton. p. 12-4.

26. King MA, Casarett GW and Weber DA, A study of irradiated bone: I. histopathologic and physiologic changes. J Nucl Med, 1979. 20(11): p. 1142-9.

27. Alwood JS, Shahnazari M, Chicana B, Schreurs AS, Kumar A, Bartolini A, Shirazi-Fard Y and Globus RK, Ionizing Radiation Stimulates Expression of Pro-Osteoclastogenic Genes in Marrow and Skeletal Tissue. J Interferon Cytokine Res, 2015. 35(6): p. 480-7.

28. Robling $A G$, Castillo $A B$ and Turner $\mathrm{CH}$, Biomechanical and molecular regulation of bone remodeling. Annu Rev Biomed Eng, 2006. 8: p. 455-98.

29. Berglundh T, Abrahamsson I, Lang NP and Lindhe J, De novo alveolar bone formation adjacent to endosseous implants. Clin Oral Implants Res, 2003. 14(3): p. 251-62.

30. Brasseur M, Brogniez V, Gregoire V, Reychler H, Lengele B, D'Hoore W and Nyssen-Behets C, Effects of irradiation on bone remodelling around mandibular implants: an experimental study in dogs. Int J Oral Maxillofac Surg, 2006. 35(9): p. 850-5. 
THE HISTOLOGICAL CHANGES AFTER IRRADIATION

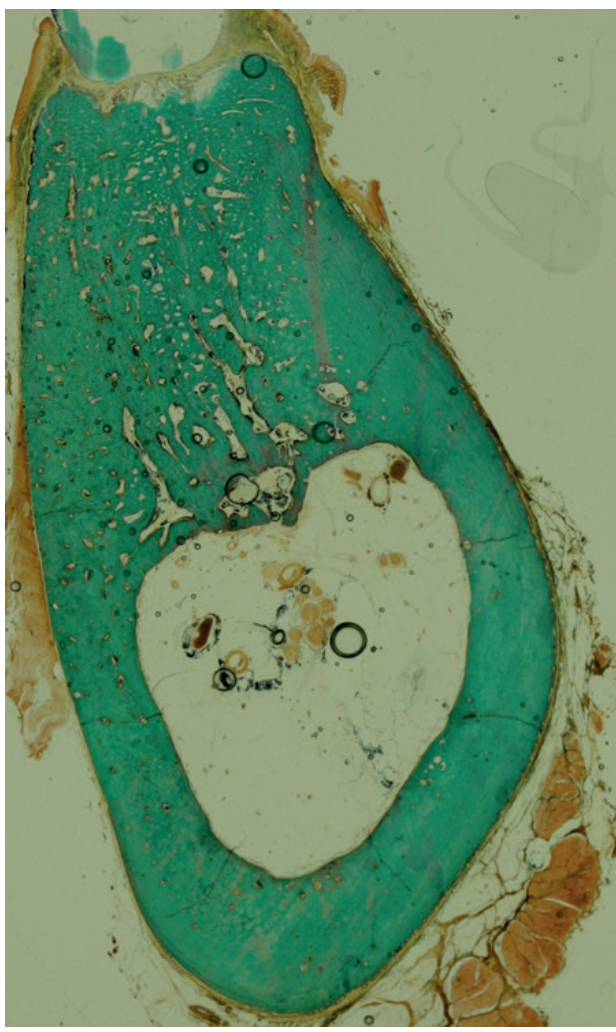

a

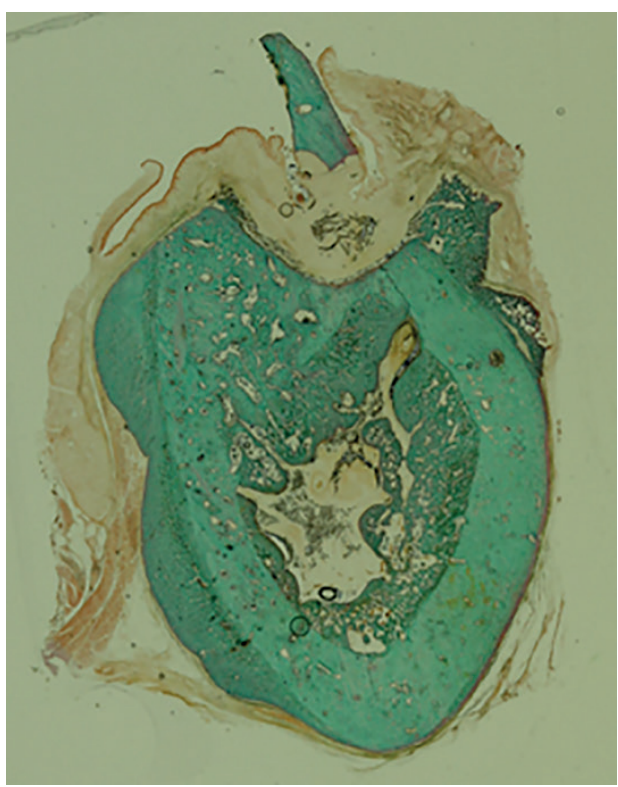

c.

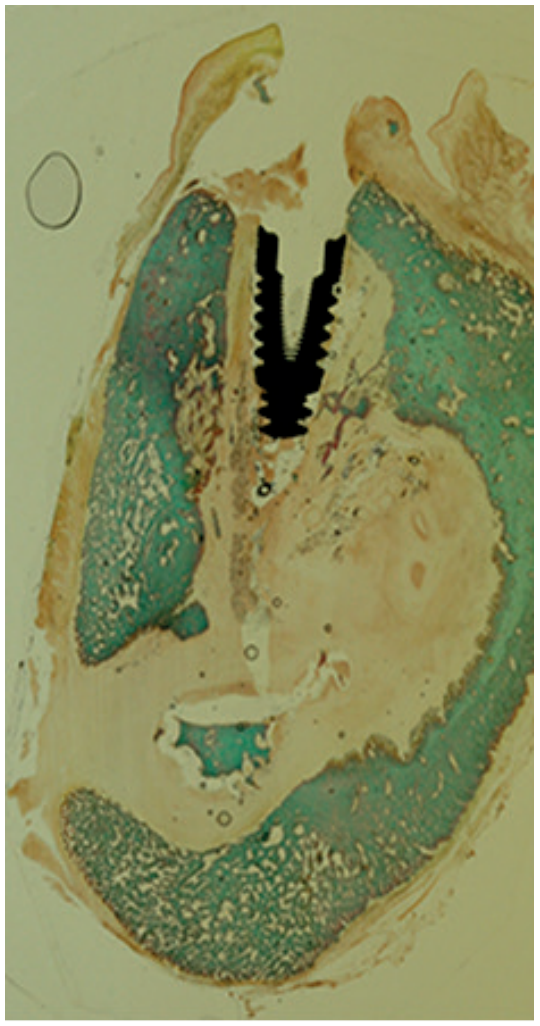

b. 


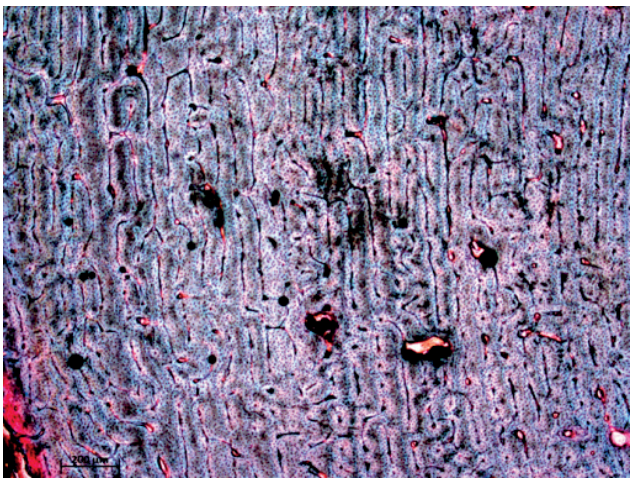

d.

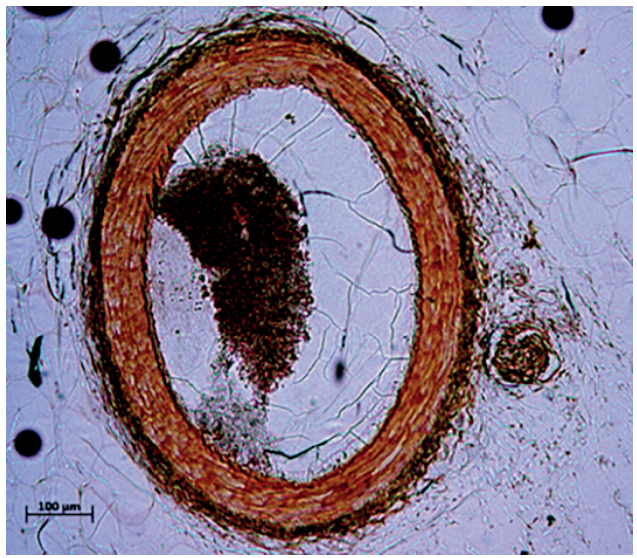

f.

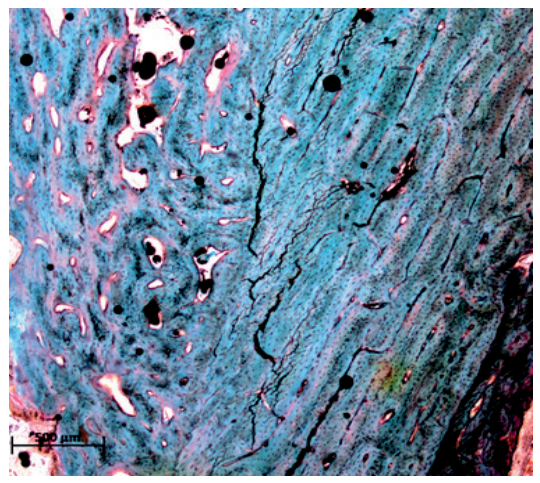

e.

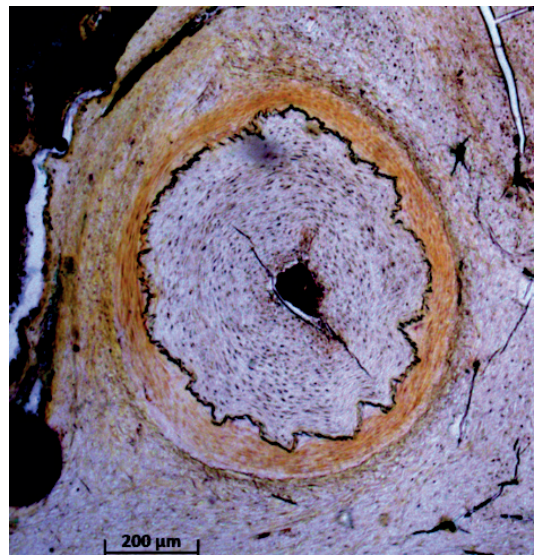

g.

Supplementary images. Mandibular bone. Figures A,B show differences in histological architecture of the mandible. A non-irradiated, $B$ and $C$ irradiated with $70 \mathrm{~Gy}$. Lacunae forming and destruction is visible. Figure $D$ (70Gy) show increased amounts of resorption lacunae, with decreased lamellar bone and increased woven bone compared to the control group (Figure E). Original magnification 25x; staining: Masson trichrome. Figure $\mathrm{G}$ (70Gy) show a decreased lumen of the IAA with a fibrotic tunica intima compared to the control group (Figure F). 


\section{CHAPTER 8}

General Discussion and Future Perspectives 



\section{GENERAL DISCUSSION}

Treatment of head and neck cancer often requires external radiation therapy (RT). Perhaps the most vexing and exigent problem confronting head and neck cancer patients is overcoming the impediments of collateral damage imposed by RT. In fact, radiation affects all the surrounding normal tissues, with different degrees of sensitivity, and may produce severe wound healing complications, such as mandibular osteoradionecrosis (ORN). ${ }^{1}$ ORN can have a severe impact on the quality of life of patients. Intraosseous vascularization is known to be decreased after irradiation, but its impact on total bone biology is still controversial. ${ }^{2-4}$

The variability of onset after RT, clinical presentation, extent and variability in progression make ORN difficult to study. In addition to the difficulties in diagnosing ORN the choice of an appropriate treatment regimen is difficult to define and may range from non-invasive measures to multiple flap micro-surgical reconstructions. ${ }^{5-10}$

The incidence of mandibular ORN in head and neck cancer patients managed with primary or adjuvant RT, varies widely in the literature from $2 \%$ to $22 \%{ }^{1}$ The interpretation of data derived from particular retrospective clinical series are difficult due to biases in patient selection, the different scoring methods and classification systems used for the evaluation of post-radiation bone damage. Although ORN occurs typically in the first three years after RT, patients probably remain at risk indefinitely. ${ }^{5,7,11}$

The consequences of RT on bone vasculature, long known to be affected by radiation, have been poorly understood. ${ }^{2,4,12}$ Therefore an appropriate animal model is needed to study both pathophysiological processes and diagnostic tools. In general, pathophysiology of ORN is caused by ongoing fibrosis, vascular compromise, and unopposed osteoclastic activity. Still it is unclear why only certain patients and not all patients develop ORN.

We performed a literature review in chapter 2 on irradiation effects on facial bones in animal experiments. The results and details in the selected studies were informative, but due to the variability of the test animals, test setup, irradiation technique and dose, and possible surgical interventions it was impossible to draw general conclusions on irradiation side effects. Recommendations on future animal research on irradiation were included in our animal model. Specific information on a semi-quantitative radiohistological scoring was found, which was used for histological evaluation, described in chapter 7. 


\section{Animal model}

ORN can occur either spontaneously or in relation with a surgical trauma. An animal model should ideally encompass both mechanisms. The animal model we developed was based on a split mouth design in which the left mandibular side was operated on in a typical oral surgical intervention, whereas the right side was left intact, and both sides were irradiated. Patients suffering from cancer in the head and neck frequently have to undergo surgical tooth sanitation or other oral surgical interventions, often followed by placements of implants. Therefore the combination of extraction and implant placement was chosen as typical intervention in our animal model. The impact of different radiation doses was studied.

The Göttingen Minipig was chosen purposely for two major reasons. First the bone regeneration rate resembles the bone regeneration of humans better than those of small test animals often used in animal experiments, such as rats or mice. The bone regeneration rate in the Göttingen Minipig is about 1.5 times higher than in humans and therefore judged as comparable. ${ }^{13}$ Secondly, the size of the minipig's mandible is ideal for experiments based on teeth extractions in combination with implant placement. $^{14}$

ORN is a complication of the bone, being a late responding tissue. ${ }^{15}$ The time needed for the total remodelling cycle of the bone for minipigs is about three months. ${ }^{16,17}$ Therefore a six month time interval for evaluation of RT effects on the bone and a three months' time interval for surgery was chosen.

The applied doses were in only two fractions due to practical limitations, and the linear quadratic model was used to calculate the equivalent dose, using an alpha beta ratio of three. ${ }^{15}$ Despite the fact that for low fractionation the exact dose effects were unsure, no animal died after irradiation and the animals reacted to irradiation as expected: two animals in the groups receiving higher irradiation doses developed typical clinical signs of ORN. We can conclude that the chosen test animal, the radiation doses applied and the RT fractionation were suitable for an animal experiment on ORN.

\section{Laser doppler flowmetry}

As described in chapter 3 our animal model enabled us to directly monitor local blood flow in irradiated and non-irradiated bone. By irradiating not only the mandible, but also the frontal bone we intended to study the blood flow in two distinctly different anatomical regions of different embryological origin.

In general there was a trend towards reduction of local tissue blood flow indirectly proportional with the increase in irradiation dose. This decrease in perfusion was more pronounced in the mandible compared to the frontal bone. The blood supply of the mandible mainly relies on the inferior alveolar artery, whereas the vasculature of the frontal bone consists of an intricate vascular web of anastomoses. ${ }^{18}$ The vascular 
architecture can partially explain the distinct vulnerability of the mandible to develop ORN, compared to the frontal bone. These findings were supported by detecting progressive fibrosis of the tunica intima of the inferior alveolar artery and a decrease in its diameter at histopathological evaluation. The relative small changes in laser doppler flowmetry measurements might be a result of the fact that the recordings were made directly after extractions causing vascular disturbances. This is in contrast with the literature, especially the results of Verdonck et al. ${ }^{19,20}$

Careful evaluation of laser doppler flowmetry measurements in the animals with clinical ORN, however, did not reveal significant differences compared to the non-ORN animals. The predictive value of flowmetry on the risk of development or even presence of ORN is limited and makes laser doppler flowmetry not suitable for detection of ORN or detection of early stages of ORN.

\section{Bone mineral density}

The bone mineral density is indirectly affected by reparative processes. Changes in bone mineral density could be an excellent non-invasive parameter for monitoring structural changes of bone after irradiation or other physico-chemical stimuli. However, the effects of irradiation on bone mineral density are not fully understood and various observations exist. Verdonck et al. found a non-significant increase in bone mineral density after irradiation of mandibular bone. ${ }^{21}$ This was confirmed by Margulies et al. and described as hypermineralisation after irradiation. ${ }^{22}$ In contrast, Fregene et al. found a decrease in bone mineral density. ${ }^{23}$ In chapter 4 we studied bone mineral density in irradiated minipig mandibles, aiming to discover a dose dependent tissue reaction. We found that bone mineral density was equally reduced in all irradiated animals and not decreased in the non-irradiated animals. The changes in bone mineral density we observed were not dose dependent. Verdonck et al found an insignificant increase in the bone mineral density after irradiation in their study of Göttingen Minipigs. All teeth were removed and the bone was allowed to heal for three months before irradiation. CT scans were performed three months after irradiation. ${ }^{21}$ Reparatory mechanisms could have caused the bone mineral density to increase slightly. Their results can be interpreted as a mechanism known from fracture healing in the long bones, especially the femoral head. There the bone density in the surrounding bony tissues increases during fracture healing. After a certain period, the bone mineral density will then return to normal. ${ }^{24}$ This final reduction of bone mineral density might have been stopped by irradiation, causing the increase compared to the non-irradiated animals.

In our study histopathological evaluation of the mandible revealed that in cortical bone there was an increase in the woven bone to lamellar bone ratio in all irradiated animals on both sides. The newly formed bone consisted of immature bone with a relatively low mineralisation grade. The observation that bone mineral density was equally decreasing 
in all irradiated animals without significant differences for those animals which revealed clinical ORN incapacitates bone mineral density measurements as a tool for quantitative analysis of bone quality after irradiation.

\section{Imaging}

The chances of radiological diagnosis of ORN by early detection of the impact of radiation on bone and vasculature received little attention in literature. In daily practice the diagnosis of ORN is based on CT and MR-imaging. CT is used for the visualisation of bony changes whereas MR-imaging can depict active bone marrow involvement in absence of tumour recurrence. ${ }^{25-27}$ Radiology, however, may enable us to study the pathophysiological process of the development of ORN in humans in vivo. ${ }^{25,28}$

In chapter 5 we based the radiological diagnosis in our animal experiment on the same non-invasive radiological methods as in humans to render translational results. Both, MR- and CT-imaging, were applied to evaluate the effects of irradiation on bone.

The two animals which met the criteria of clinical ORN, were diagnosed as ORN with MR- and CT- imaging. In addition a third animal with subclinical or healed ORN was detected by radiological imaging and confirmed by histopathologic evaluation. Because of the relative short observation time this was more probably ORN in progress than healing although no clinical observations were done during the 3 months after surgery. The ORN animals showed cortical destruction of the mandible visible on CT and bone marrow involvement with an increase in water signal at all sequences at MR-imaging resembling bone marrow edema patterns. The T2-SPIR sequence however demonstrated a mixed pattern in the clinical ORN cases. This advocates for inclusion of this sequence in the routine clinical diagnostic work-up for ORN. The mixed bone marrow edema pattern reflects the active pathophysiological process of ORN, with both fields of inflammation and fibrosis. Since this process is responsible for the development of clinical ORN, definition and detection of pre-ORN marrow conditions could aid early medical intervention, possibly preventing ORN or reducing the severity of ORN. At higher irradiation dose, an increase in water content was noted on T1, T2, STIR and post Gadolineum sequences. Histopathological evaluation showed increased presence of fibrosis and cortical resorption lacunae in higher irradiation doses parallel to findings of altered signal intensities on MR-imaging.

Dual energy CT (DE-CT) allows for filtering calcium containing tissues enabling the study of the soft tissues of the bone. DE-CT derived virtual non-calcium images were studied as an alternative method to detect bone marrow edema in vertebrae, the ankle and knee, ${ }^{29-31}$ but it has not yet been tested in a more complex anatomical structure such as the mandible. In chapter 6 we used this diagnostic tool to explore the degree of bone marrow edema, as we believe that the cause of the development of ORN is based on a preceding edema and demineralisation process. Edema of the bone marrow is found 
after irradiation ${ }^{25,26}$ and might be caused by a prolonged regeneration process after irradiation. The virtual non-calcium images compared to the edema found on MRimaging demonstrated a good correlation between HU-values and STIR signal intensity, indicating edema at higher values and hyperintensity at STIR-imaging. Virtual nonCalcium images offer the opportunity to be used for the detection of bone marrow edema of the mandible and more preferably for early detection of ORN and pre-ORN conditions.

\section{Histopathological evaluation}

In chapter 7 we studied histological and histomorphometric alterations in healthy bone in our large animal experiment on Göttingen minipigs. Irradiation causes a compromised vascularisation, due to fibrosis of the vascular walls, especially the tunica intima of the arteries. This pathophysiological process leads to hypoperfusion and demineralisation. The regenerative capacity of irradiated tissues resulted in insufficient bone regeneration with an increased amount of newly formed disorganised bone at a reduced remodelling speed. The woven bone/lamellar bone ratio increased depending on the irradiation dose. The mandibular bone specimens of all irradiated animals showed these changes in a varying degree. Three animals developed ORN histopathologically, revealing bone sequesters in two and extensive destruction of cortex in all three. The deterioration of bone quality and a compromised vascularisation on itself does not necessarily lead to the clinical diagnosis of ORN. We think the key to develop ORN lies in the unopposed osteoclastic activation also found by Störe et al. ${ }^{4}$ possibly triggered by local hypoxia. ${ }^{4,32}$ We did find increase in osteoclastic activity with increased irradiation dose seen as an increase in resorption lacunae, especially in association with surgical interventions. In humans there is a specific genetic allotype for TGF- $\beta 1$ receptor resulting in an increased risk for ORN found by Lyons et al. ${ }^{33}$ This receptor change causes further increase in fibrosis and possibly explains why not all patients and not all animals develop ORN.

The limitation of this experimental model is the fact that an animal model had to be chosen to describe side effects of irradiation in the head and neck field. However close the animal's bone biology resembles the situation in humans it will always be somewhat different. We were able to analyse histology, radiology and experimental methods to evaluate the effects of various irradiation doses on healthy bone with controlled variables, increasing the strength of the thesis. As described by Marx et al. ${ }^{2}$ ORN has an early and late form. Since the observation time was limited due to logistic reasons the irradiation effects observed must be regarded as early or acute effects of irradiation. The animal model as defined in this study seems to be ideal for further research on ORN. 


\section{Conclusion}

The results of this experimental research added to the knowledge on the pathophysiology and development of ORN in the head and neck, as well as to the diagnostic necessities to detect ORN in preclinical stages.

MR-, conventional CT- and DE-CT-imaging are useful for the diagnosis and early detection of ORN. Cortical destruction was noted on CT- and on MR-imaging, specifically T2-SPIR displayed a specific signal intensity pattern in the clinical ORN cases. In general increased signal intensity changes at T2-weighted sequences were seen with increased irradiation doses, resembling bone marrow edema. Mixed signal intensities on T2-SPIR after RT were exclusively found in the clinical ORN cases and visualize the pathophysiological process related to the development of ORN. DE-CT derived virtual non-Calcium images correlated to MR-imaging edema patterns and therefore DE-CT can be regarded as a feasible imaging tool to replace MR-imaging in the future. The diagnostic tools mentioned above offer an opportunity to create a new gradation system for the amount of radiation damage and loss of bone quality.

The development of ORN requires three premises: firstly vascular fibrosis leading to decreased blood flow, secondly decreased bone remodelling and disorganised bone repair and thirdly increased osteoclastic activity. On the cellular level unopposed osteoclastic activity possibly triggered by hypoxia may be the fundamental biological process. 


\section{FUTURE PERSPECTIVES}

RT is without doubt beneficial in the treatment of head and neck cancer patients. In view of the possible side effects careful patient selection and protection from these undesirable effects remain an absolute necessity. Tailor made treatment planning in head and neck cancer includes refinement of RT equipment, protocols and treatment planning. Apart from this, further research is needed to identify patient specific genetic risk factors for developing ORN. ${ }^{33}$ Intensified follow-up of RT-side effects on bone quality, should prevent ORN and warrant patients' quality of life after treatment of head and neck malignancies for the rest of their lives.

Further research regarding drug treatment of ORN is necessary. Since the animal model used in our study proved highly comparable to the human situation, this study model offers ideal opportunities to examine the effects of drugs on ORN. Based on the possible mechanism of unopposed osteoclastic activity special interest should be directed to investigate the benefits of Clodronate, since it inhibits osteoclasts and also stimulates osteoblast activity.

Future clinical studies should aim at further improvement and clinical validation of Dual Energy CT derived virtual non-calcium images for the early detection of bone marrow edema in preclinical ORN, as an integral tool in the regular follow-up program of irradiated head and neck cancer patients. Dual energy CT could replace the combination of conventional CT- and MR-imaging for the diagnosis of ORN and help to avoid development of clinical ORN. Mixed images from dual energy CT could lead to patient specific evaluation of bone quality, thus improving personalized treatment in this highrisk patient group. 


\section{REFERENCES}

1. Teng MS and Futran ND, Osteoradionecrosis of the mandible. Curr Opin Otolaryngol Head Neck Surg, 2005. 13(4): p. 217-21.

2. Marx RE and Johnson RP, Studies in the radiobiology of osteoradionecrosis and their clinical significance. Oral Surg Oral Med Oral Pathol, 1987. 64(4): p. 379-90.

3. Delanian $\mathrm{S}$ and Lefaix $\mathrm{JL}$, Complete healing of severe osteoradionecrosis with treatment combining pentoxifylline, tocopherol and clodronate. Br J Radiol, 2002. 75(893): p. 467-9.

4. Store $G$ and Granstrom G, Osteoradionecrosis of the mandible: a microradiographic study of cortical bone. Scand J Plast Reconstr Surg Hand Surg, 1999. 33(3): p. 307-14.

5. Notani K, Yamazaki Y, Kitada H, Sakakibara N, Fukuda H, Omori K and Nakamura M, Management of mandibular osteoradionecrosis corresponding to the severity of osteoradionecrosis and the method of radiotherapy. Head Neck, 2003. 25(3): p. 181-6.

6. Annane D, Depondt J, Aubert P, Villart M, Gehanno P, Gajdos P and Chevret S, Hyperbaric oxygen therapy for radionecrosis of the jaw: a randomized, placebo-controlled, double-blind trial from the ORN96 study group. J Clin Oncol, 2004. 22(24): p. 4893-900.

7. Gevorgyan A, Wong K, Poon I, Blanas N, Enepekides DJ and Higgins KM, Osteoradionecrosis of the mandible: a case series at a single institution. J Otolaryngol Head Neck Surg, 2013. 42: p. 46.

8. Robard L, Louis MY, Blanchard D, Babin E and Delanian S, Medical treatment of osteoradionecrosis of the mandible by PENTOCLO: Preliminary results. Eur Ann Otorhinolaryngol Head Neck Dis, 2014.

9. Delanian S, Depondt J and Lefaix JL, Major healing of refractory mandible osteoradionecrosis after treatment combining pentoxifylline and tocopherol: a phase II trial. Head Neck, 2005. 27(2): p. 114-23.

10. Jacobson AS, Buchbinder $\mathrm{D}, \mathrm{Hu} \mathrm{K}$ and Urken ML, Paradigm shifts in the management of osteoradionecrosis of the mandible. Oral Oncol, 2010. 46(11): p. 795-801.

11. Epstein JB, Wong FL and Stevenson-Moore P, Osteoradionecrosis: clinical experience and a proposal for classification. J Oral Maxillofac Surg, 1987. 45(2): p. 104-10.

12. Delanian $\mathrm{S}$ and Lefaix JL, The radiation-induced fibroatrophic process: therapeutic perspective via the antioxidant pathway. Radiother Oncol, 2004. 73(2): p. 119-31.

13. Pearce Al, Richards RG, Milz S, Schneider E and Pearce SG, Animal models for implant biomaterial research in bone: a review. Eur Cell Mater, 2007. 13: p. 1-10.

14. Saka B, Wree A, Anders L and Gundlach KK, Experimental and comparative study of the blood supply to the mandibular cortex in Gottingen minipigs and in man. J Craniomaxillofac Surg, 2002. 30(4): p. 219-25.

15. Fowler JF, 21 years of biologically effective dose. Br J Radiol, 2010. 83(991): p. 554-68.

16. Clarke B, Normal bone anatomy and physiology. Clin J Am Soc Nephrol, 2008. 3 Suppl 3: p. S131-9.

17. Martinez-Gonzalez JM, Cano-Sanchez J, Campo-Trapero J, Gonzalo-Lafuente JC, Diaz-Reganon J and Vazquez-Pineiro MT, Evaluation of minipigs as an animal model for alveolar distraction. Oral Surg Oral Med Oral Pathol Oral Radiol Endod, 2005. 99(1): p. 11-6.

18. Carlson B, Embryology \& Developmental Biology. Mosby., 1999: p. 166-170.

19. Verdonck HW, Meijer GJ, Laurin T, Nieman FH, Stoll C, Riediger D, Stoelinga PJ and de Baat C, Assessment of vascularity in irradiated and nonirradiated maxillary and mandibular minipig alveolar bone using laser doppler flowmetry. Int J Oral Maxillofac Implants, 2007. 22(5): p. 774-8.

20. Verdonck HW, Meijer GJ, Kessler P, Nieman FH, de Baat C and Stoelinga PJ, Assessment of bone vascularity in the anterior mandible using laser Doppler flowmetry. Clin Oral Implants Res, 2009. 20(2): p. 140-4.

21. Verdonck HW, Meijer GJ, Nieman FH, Stoll C, Riediger D and de Baat C, Quantitative computed tomography bone mineral density measurements in irradiated and non-irradiated minipig alveolar bone: an experimental study. Clin Oral Implants Res, 2008. 19(5): p. 465-8.

22. Margulies B, Morgan H, Allen M, Strauss J, Spadaro J and Damron T, Transiently increased bone density after irradiation and the radioprotectant drug amifostine in a rat model. Am J Clin Oncol, 2003. 26(4): p. e106-14. 
23. Fregene $A$, Jing $X L$, Monson LA and Buchman SR, Alteration in volumetric bone mineralization density gradation patterns in mandibular distraction osteogenesis following radiation therapy. Plast Reconstr Surg, 2009. 124(4): p. 1237-44.

24. lida $Y$, Kuroda $T$, Kitano $T$ and Mizuno $K$, Dexa-measured bone density changes over time after intertrochanteric hip fractures. Kobe J Med Sci, 2000. 46(1-2): p. 1-12.

25. Bachmann G, Rossler R, Klett R, Rau WS and Bauer R, The role of magnetic resonance imaging and scintigraphy in the diagnosis of pathologic changes of the mandible after radiation therapy. Int J Oral Maxillofac Surg, 1996. 25(3): p. 189-95.

26. Tartaglino LM, Rao VM and Markiewicz DA, Imaging of radiation changes in the head and neck. Semin Roentgenol, 1994. 29(1): p. 81-91.

27. Hermans R, Fossion E, loannides C, Van den Bogaert W, Ghekiere J and Baert AL, CT findings in osteoradionecrosis of the mandible. Skeletal Radiol, 1996. 25(1): p. 31-6.

28. Store G, Smith HJ and Larheim TA, Dynamic MR imaging of mandibular osteoradionecrosis. Acta Radiol, 2000. 41(1): p. 31-7.

29. Wang CK, Tsai JM, Chuang MT, Wang MT, Huang KY and Lin RM, Bone marrow edema in vertebral compression fractures: detection with dual-energy CT. Radiology, 2013. 269(2): p. 525-33.

30. Pache G, Krauss B, Strohm P, Saueressig U, Blanke P, Bulla S, Schafer O, Helwig P, Kotter E, Langer M, and Baumann T, Dual-energy CT virtual noncalcium technique: detecting posttraumatic bone marrow lesions--feasibility study. Radiology, 2010. 256(2): p. 617-24.

31. Guggenberger R, Gnannt R, Hodler J, Krauss B, Wanner GA, Csuka E, Payne B, Frauenfelder T, Andreisek $\mathrm{G}$ and Alkadhi $\mathrm{H}$, Diagnostic performance of dual-energy $\mathrm{CT}$ for the detection of traumatic bone marrow lesions in the ankle: comparison with MR imaging. Radiology, 2012. 264(1): p. 164-73.

32. Carmeliet $P$ and Jain RK, Molecular mechanisms and clinical applications of angiogenesis. Nature, 2011. 473(7347): p. 298-307.

33. Lyons AJ, West CM, Risk JM, Slevin NJ, Chan C, Crichton S, Rinck G, Howell D and Shaw RJ, Osteoradionecrosis in head-and-neck cancer has a distinct genotype-dependent cause. Int J Radiat Oncol Biol Phys, 2012. 82(4): p. 1479-84. 

Valorisation addendum 


\section{INTRODUCTION}

In the western world, head and neck cancer constitutes approximately $5 \%$ of all cancers. In the Netherlands, head and neck cancers account for 2500 new cancer cases annually. For head and neck malignancies, current treatment regimens can include radiotherapy (RT). RT has known beneficial effects both as a primary and as a secondary treatment, i.e., postoperative RT after surgical resection. However, despite improving and refining radio-therapeutic techniques, such as intensity-modulated radiation therapy (IMRT), irradiation effects on the surrounding healthy tissue continue to cause complications.

One of the known complications of RT is osteoradionecrosis (ORN), which can affect all bony structures in the field of irradiation, but is most commonly seen in the mandible at a frequency of $2-22 \%$. The diagnosis is generally based on a clinical presentation. ORN is defined as "irradiated bone, which becomes devitalised and is exposed through the overlying skin or mucosa and does not heal within a period of three months, without tumour recurrence". Several factors have been identified that increase the risk of developing ORN including treatment-related factors, such as the radiation dose and the volume of irradiation. ORN can be triggered by surgical intervention or pressure sores from dentures and can occur spontaneously. It can arise at any point after irradiation therapy even several years later.

Treatment of ORN ranges from conservative management with or without surgical debridement to wide resections in severe cases. Due to the compromised local tissue condition, reconstruction with micro-surgical free-flap transfer is the standard treatment. This is a major surgical intervention in patients with severe comorbidities. Functional and aesthetic limitations are inevitable and dramatically reduce the quality of life of the affected patients.

Between 2007 and 2013, our head and neck team at the MUMC+ treated approximately 1200 new oncologic cases of which an estimated 300 cases received partial irradiation of the mandible. We recorded 15 cases of ORN, which gives an estimated frequency of $5 \%$. This figure is comparable to the incidences mentioned in the literature.

Due to the variability in onset and presentation as well as the variable progression of mandibular ORN, clinical studies are difficult to perform. The exact pathophysiological mechanisms leading to the development of ORN are not yet fully understood, and therefore, a targeted treatment strategy is lacking.

To study the pathophysiological conditions leading to ORN, we developed a large animal model. With regard to bone physiology, the Göttingen minipig has anatomical and biological characteristics highly comparable to human beings. This enabled us to study the pathophysiological processes that are similar to the human situation under controlled conditions. Increasing irradiation doses were applied with and without surgical interventions. Moreover, imaging to assess mandibular ORN has received little attention in the literature. The added value of the visualisation of the pathophysiological processes, however, is evident. 


\section{RESULTS}

The animal model and experiments described in this thesis are realistic, because three of the twelve irradiated animals developed ORN using a radiation pattern with two fractions. Another research group irradiated minipigs with only one fraction, and all animals developed ORN. However, in our study, only one in four animals developed ORN. Therefore, the radiation pattern based on only two fractions resulted in clinically comparable outcomes with respect to ORN.

Using laser Doppler flowmetry, we found a non-significant progressive decrease in the local blood flow. This was confirmed histologically, where a decreased vascular diameter due to intima fibrosis could be demonstrated. Using conventional CT imaging, we found a non-progressive decrease in bone mineral density after irradiation, which was likely caused by prolonged and insufficient remodelling after irradiation.

All animals underwent both MR- and CT-imaging. In the ORN animals, cortical destruction and marrow involvement were present. With an increase in the irradiation dose, MR-imaging revealed progressive bone marrow oedema due to the irradiation on both the surgical and non-surgical side. Furthermore, there was a considerable variance between the animals from the same irradiation groups. One animal had no clinical signs of ORN, but had radiological ORN and histological changes; these variable results emphasise the need for imaging.

Additional imaging was performed using dual energy CT (DE-CT). With this technique the evaluation of the bone marrow is made possible using CT. We compared the density of the bone marrow on virtual non-calcium images to the signal intensity of the bone marrow from the STIR images. We found that the DE-CT images were comparable to the MR-imaging for the detection of the marrow oedema pattern. This is potentially a new application for the detection of ORN in patients after clinical validation.

Histological evaluation revealed progressive fibrosis in general and especially in the vascular wall of the inferior alveolar artery. Necrosis and bone destruction were clearly visible in the ORN cases. The bone remodelling was markedly decreased with an increase in the irradiation dose.

Based on the findings of our animal model, we can conclude that the pathophysiological process leading to ORN is a variable process; therefore not all animals developed ORN.

ORN appears to be caused by vascular compromise combined with reduced and insufficient bone regeneration.

\section{CLINICAL IMPLICATIONS}

The specific groups that will benefit from this study are head and neck cancer patients receiving $\mathrm{RT}$ and the multidisciplinary team involved in the treatment of head and neck cancer. With renewed attention to the problematic complication of ORN and the need 
for careful planning of the RT, early diagnosis and treatment and intensified follow-up after RT are necessary. We expect that such an approach can improve the patient quality of life after radiotherapy for head and neck cancer. Hopefully early detection of preclinical ORN using CT- and MR-imaging can prevent the progression of the disease using medical treatment, such as treatment with pentoxyphilin, tocopherol and clodronate.

\section{SHARING KNOWLEDGE}

The knowledge gained by this study will be shared both by means of scientific publications in peer-reviewed journals and in presentations at national and international congresses. Furthermore, the intensified collaboration with the departments of radiology and radiotherapy will increase the multidisciplinary awareness of ORN. The results will be shared to the entire multidisciplinary head and neck oncology team; therefore, patients will benefit from these findings.

\section{FUTURE PERSPECTIVES}

To prevent the development of ORN, investigations should focus on further improvements and refinements in radiotherapy for head and neck cancer. Second, to detect preclinical ORN and to initiate immediate medical treatment, regular and intensified follow-up of irradiated patients is necessary. This preclinical detection would prevent the worsening of ORN and the deterioration of the patient's quality of life after radiotherapy.

In addition to improvements in clinical detection, future studies should aim for clinical validation and implementation of the dual energy CT technique for detecting ORN in human subjects. The use of this technology would make the diagnostic work-up for the early detection of ORN easier and faster.

Dual energy CT might also be a promising tool for assessing bone quality after irradiation prior to surgical procedures in the mandible or during follow-up. 
SUMMARY 
Chapter 1 introduction, describes the application of radiotherapy (RT) for head and neck malignancies and its beneficial effects on the time to recurrence and survival. However, a serious and late complication of RT is osteoradionecrosis (ORN), most commonly seen in the mandible. ORN can range from a mild to severe entity, the latter needs a microsurgical reconstruction. Pathophysiology of ORN involves vascular compromise, and dysregulated fibroblastic activity. However, the exact pathophysiological mechanism for the development of ORN remains unclear. Radiological diagnosis of ORN constitutes of MR- and CT-imaging, which until now it received little attention in literature. Bone changes and vascular effects after irradiation have been described but general consensus on these effects is lacking.

The aim of this thesis was to study the pathophysiological conditions leading to ORN in a large animal model.

In Chapter 2 a literature search on the experimental irradiation of facial bones in animals was conducted. The search was performed on publications between January 1992 and January 2012, with the search terms: "irradiation facial bone" and "irradiation osteoradionecrosis". A total of 36 publications were included. The irradiation sources were Cobalt 60, orthovoltage, 4 - 6 Megavolt photons, and brachytherapy. The total dose varied between 8 - $60 \mathrm{~Gy}$ in single or multiple fractions. The included studies presented a broad range of animal studies that differed in terms of the in vivo model, irradiation, observation period, and evaluation of results. This diversity in methods and end-points hampers the interpretation of results. This led to the conclusion that development and implementation of a detailed and standardized description of the methodology and results would facilitate the comparability of future studies.

In Chapter 3 the effects of various doses of irradiation on blood flow in the mandibular bone were compared to the os frontale. In 16 female Göttingen minipigs the mandibles and os frontale were irradiated with equivalent doses of 0, 25, 50 and 70 Gray. 3 months after irradiation, mandibular left premolars and molars were removed and dental implants were placed. 3 months after irradiation laser doppler flowmetry (LDF) was used to record local blood flow on the left mandible and in the irradiated area of the os frontale. At 6 months after irradiation, LDF measurements were repeated. Local blood flow measurements in the left hemi-mandible, were significantly higher compared to the os frontale. At 3 and 6 months, values of both os frontale and mandible showed no significant differences in the different dose groups. However a non-significant decrease in LDF values with an increase in irradiation dose in the mandible after both 3 and 6 months was visible.

In Chapter 4 changes in mandibular bone mineral density after irradiation in various doses with and without surgery were investigated. CT-scans were made before and 6 months after irradiation. The measured bone density was related to a bone phantom to 
calculate the bone mineral density quotient (BMDQ). We found a significant decrease on both sides in the irradiated animals, but no significant correlation could be found between the irradiation dose and a decrease in BMDQ.

Chapter 5 describes radiological changes in mandibular bone after irradiation with various doses with and without surgery in order to determine imaging characteristics of radiotherapy induced changes and ORN in our animal model. 16 Göttingen minipigs had CT- and MR-imaging made before irradiation and 6 months after irradiation. The MRimaging protocol consisted of T1, T2-TSE and T2-SPIR, STIR-images and post-gadolinium T1-weighted images. Alterations in the bony structures were recorded on CT- and MRimaging and scored by 2 head-neck radiologists.

2 animals developed ORN clinically. Radiologically mixed signal intensities on T2-SPIR were seen. On CT-scans cortical destruction was found in 3 animals. Based on imaging, 3 animals were diagnosed with ORN. Additionally, we found that higher radiation dose at the mandible was associated with an increased signal intensity at T2-weighted and STIR images, consistent with bone marrow edema pattern.

Imaging with CT- and MR-imaging showed signal and structural changes that can be interpreted as prolonged and insufficient repair of radiation induced bone damage. Whereas clinically only exposed bone can be appreciated in ORN, CT- and MR-imaging facilitates visualisation of submucosal changes, thus adding to the accuracy and early detection of beginning or latent ORN.

In Chapter 6 the accuracy of Dual Energy CT (DE-CT) for detection of edema of the mandible after irradiation and surgery was compared to MR-imaging. In the same experimental setup, 15 of the animals underwent DE-CT and MR-imaging of the mandible 6 months after radiation. MR STIR was used for grading of bone marrow edema, on a scale of 0 to 3. DE-CT with simultaneous imaging at $80 \mathrm{kVp}$ and $140 \mathrm{kVp}$ was performed and virtual non-calcium (VNCa) images were calculated. HU-values were measured by 2 observers and compared to MR-imaging. STIR demonstrated increased signal intensity in the higher radiation groups. An increase of signal intensity at STIR imaging was accompanied by a significant increase in HU-value at VNCa images in the bone marrow.

Thus, VNCa images derived from DE-CT were able to demonstrate bone marrow edema in radiation induced bone changes in the mandible. So in addition to the ability of CT to depict cortical destruction and trabecular bone resorption, DE-CT VNCa images can provide additional information on the presence of bone marrow edema in one stop.

Chapter 7 reports on the histological and histomorphometric changes induced by external irradiation in our animal experiment. In this experiment, to study bone apposition, fluorochrome markers were subcutaneously administered at 2, 14, and 24 weeks after irradiation. The pigs were sacrificed at 26 weeks after irradiation, and the 
mandibular bone samples were stained with Masson's trichrome and semiquantitatively analyzed.

The amount of fibrosis, necrosis, resorption lacunae and the ratio woven/lamellar bone were increased after higher radiation dosages and surgery. The inferior alveolar artery was affected by radiotherapy. The vascular diameter was reduced, the tunica intima, and the tunica media-intima thickness were increased depending on the irradiation dosages. In general, the bone remodeling rate was decreased after irradiation. Surgical trauma could increase bone remodeling rates in all groups locally. Both surgery and increasing irradiation dosages cause architectural bone changes and damage the vascularization. This might result in a chronic hypoxic state of the mandibular bone. This state could be prerequisite to the development on ORN

In Chapter 8 above findings were discussed. A decrease of vascularity and bone mineral density with increased irradiation dose, were noted at histopathological evaluation. This was however not appreciated with the experimental diagnostic tools as were bone mineral density and laser doppler flowmetry measurements. In conclusion bone mineral density measurements and laser Doppler flowmetry were not found to be valuable diagnostic tools for the detection of bone quality changes after irradiation and ORN.

Imaging plays an important role in the diagnostics of ORN. Cortical disruption is best noted with $\mathrm{CT}$, while bone marrow changes can best be appreciated with MR-imaging. CT- and MR-imaging were able to detect the clinical ORN cases, as well as a preclinical case of ORN, as was confirmed histologically. The mixed signal intensity at T2-SPIR imaging was solely appreciated in clinical ORN cases, making this sequence a prerequisite in the diagnostic MR armentarium.

At higher radiation dose an increase in bone marrow edema pattern was noted at MRimaging. This increase in bone marrow edema pattern could be visualized with DE-CT derived virtual non-calcium images and compared to MR-imaging. This offers the propensity for one stop shop imaging of ORN and radiotherapy induced changes of the mandible with DE-CT.

The pathophysiology of irradiation damage of the mandible leading to ORN consists of three components: firstly, vascular fibrosis leading to decreased blood flow, secondly decreased bone remodelling and disorganized architecture and thirdly unopposed osteoclastic activity. We believe that when the fragile balance of these processes is lost, the critical threshold of hypoxia is passed and ORN can develop.

\section{Valorisation}

Due to the variability in onset and presentation, as well as the variable progression of mandibular ORN, the clinical study of ORN in the human population is challenging. The 
ambiguous predictability of treatment outcome and the difficulties studying the effect of treatment of ORN is complicated by the variability of ORN.

In order to study the pathophysiological conditions leading to ORN we developed a large animal model to study pathophysiological processed comparable to the human situation under controlled conditions with increasing irradiation doses. The application of various diagnostic modalities, make the findings of our study of major interest and gives a clear translation of our findings to the human situation.

Future studies should aim at identifying patient specific risk factors for development of ORN and possible drug treatment of ORN. Furthermore, the use of modern imaging techniques like DE-CT should aim at refinement of the protocols and clinical validation in patients at risk for ORN. 

SAMENVATTING 
Hoofdstuk 1, de inleiding, beschrijft de toepassing van radiotherapie (RT) voor de behandeling van hoofd- en hals tumoren en de gunstige effecten op de recidief kans en overleving. Echter een ernstige en late complicatie van de RT is osteoradionecrose (ORN), dit treft meestal de mandibula. De ernst van ORN varieert van een milde tot een complexe vorm, deze laatste wordt behandeld met resectie en micro-chirurgische reconstructie.

De hypothese van de pathophysiologie van ORN omvat een verminderde doorbloeding en ontregeling van de fibroblasten activiteit, maar de precieze oorzaak van het ontwikkelen van ORN blijft onduidelijk.

De radiologische diagnose van ORN wordt gesteld middels MR- en CT scans, maar kreeg maar weinig aandacht in de recente literatuur. Ook pathophysiologische veranderingen van botweefsel en vasculaire structuren door bestraling zijn beschreven maar er mist een algemene consensus over deze effecten.

Het doel van dit proefschrift is om de pathophysiologische processen welke leiden tot het ontstaan van ORN te bestuderen in een groot dier model.

In hoofdstuk 2 werd een literatuuronderzoek gedaan naar experimenten van bestraling van aangezichtsbeenderen in dieren. De periode januari 1992 tot en met januari 2012 werd doorzocht met de zoektermen: "irradiation facial bone" en "irradiation osteoradionecrosis". In totaal werden 36 publicaties geïncludeerd. De bestralingsbronnen waren Cobalt 60, orthovoltage, 4 - 6 megavolt fotonen, en brachytherapie. De totale dosis varieerde van 8 - $60 \mathrm{~Gy}$ in enkele of meerdere fracties. De geïncludeerde studies presenteerden een breed scala aan dierstudies die verschilden in model, bestraling, observatieperiode en evaluatie van de uitkomsten. De verschillende gepubliceerde diermodellen lieten veel vragen onbeantwoord en waren lastig vergelijkbaar. Een gedetailleerde en gestandaardiseerde manier van uitvoeren en beschrijven van methoden en resultaten in toekomstige studies zal in theorie het vergelijken van dergelijke studies vergemakkelijken.

In hoofdstuk 3 werden de effecten van oplopende bestralingsdoses op de lokale doorbloeding in de mandibula vergeleken met het os frontale. Van 16 vrouwelijke Göttingen minivarkens werden de mandibula en het os frontale bestraald, met de equivalent doses van 0, 25, 50 en 70 Gy. 3 maanden na de bestraling werden de linker premolaren en molaren van de mandibula verwijderd en werden dentale implantaten geplaatst. Drie maanden na de bestraling werd ook met laser doppler flowmetry (LDF) de lokale doorbloeding gemeten in de mandibula en het os frontale. Zes maanden na de bestraling werden de LDF metingen herhaald. De doorbloedingsmetingen in de linker mandibula waren significant hoger dan inhet os frontale. Zowel bij 3 als bij 6 maanden waren er zowel van de mandibula als het os frontale geen significante verschillen tussen de verschillende dosis groepen. Er was echter wel een niet significante daling in de LDF- 
waarden bij een toename van de bestralingsdosis van de mandibula, zowel 3 als 6 maanden na de bestraling.

In hoofdstuk 4 werden de veranderingen in de botdichtheid van de mandibula na bestraling met verschillende doses en met en zonder chirurgische interventie onderzocht. Er werden CT scans gemaakt vóór en 6 maanden na de bestraling. De meting van de botdichtheid werd gedeeld door de meting van de dichtheid van een bot fantoom, welke de botdichtheidsquotiënt (BMDQ) gaf. Er was een significante daling van BMDQ aan beide zijden van de mandibula in de bestraalde dieren, er was echter geen significante correlatie tussen deze daling en de bestralingsdosis.

Hoofdstuk 5 beschrijft radiologische veranderingen van het bot van de mandibula na bestraling met verschillende doses met en zonder chirurgische interventie. Dit om karakteristieke veranderingen na bestraling en ORN vast te stellen in ons diermodel. Dezelfde 16 Göttingen minivarkens als boven beschreven kregen een MR- en CT scan voor de bestraling en 6 maanden na de bestraling. Het MRI protocol bestond uit de volgende sequenties: T1-gewogen, T2-TSE en T2-SPIR, STIR en T1 post-gadolinium. Veranderingen in de benige structuren werden vastgelegd en gescoord door 2 hoofdhals radiologen.

2 dieren ontwikkelde klinisch ORN. Radiologisch was bij deze dieren gemengde signaalintensiteit te zien op de T2-SPIR beelden. Op de CT beelden was er in 3 dieren botdestructie zichtbaar. Gebaseerd op de beeldvorming werd bij 3 dieren de diagnose ORN vastgesteld. In het algemeen zagen we bij de dieren uit de hogere bestralingsdoses groepen geassocieerde sterke beenmerg signaal afwijkingen.

Beeldvorming met MR- en CT-scans liet zowel signaal als ook structurele afwijkingen zien welke geïnterpreteerd kunnen worden als vertraagd en onvolledig herstel van de door de bestraling veroorzaakte botdestructie. Waar klinisch alleen de diagnose gesteld kan worden op bloot liggend bot, kunnen CT- en MR-scans de submucosale veranderingen in beeld brengen. Dit helpt bij het accuraat en vroegtijdig detecteren van beginnende en latente ORN.

In hoofdstuk 6 wordt de bruikbaarheid van Dual Energy CT (DE-CT) voor de detectie van oedeem van de mandibula na bestraling en chirurgische interventie bepaald door te vergelijken met MR-beelden. In dezelfde experimentele setup als boven beschreven werd 6 maanden na bestraling bij 15 dieren naast de MR-scan ook een DE-CT-scan verricht van de mandibula. Van de MR-scan werd de STIR sequentie gebruikt voor gradering van het oedeem van het beenmerg van de mandibula op een schaal van 0 tot 3.

Met postprocessing werden virtuele non-Calcium (VNCa) beelden berekend uit de DECT data. De HU-waardes in het beenmerg en de cortex, werden vergeleken met de scores van de MR-scans. De STIR-sequentie toonde een verhoogde signaal intensiteit in 
de hogere bestralingsgroepen. De toename in signaal intensiteitsscore op MR-scan ging gepaard met een significante verhoging van de HU-waarde gemeten op de VNCa beelden ter plaatse van het beenmerg. Hierop werd geconcludeerd dat de VNCa beelden afgeleid van DE-CT-scan beenmerg oedeem kunnen aantonen na bestraling van de mandibula. Naast het afbeelden van benige afwijkingen als corticale destructie en resorptie van het trabeculair bot, kunnen DE-CT afgeleide VNCa beelden ook informatie geven over de aanwezigheid van oedeem in één enkele scan sessie.

Hoofdstuk 7 beschrijft de histologische en histomorfometrische veranderingen na bestraling in ons diermodel. De bot appositie werd gemarkeerd met fluorochroom markers welke subcutaan werden toegediend 2, 14 en 24 weken na de bestraling. $\mathrm{Na}$ het opofferen van de dieren 26 weken na de bestraling, werden de bot coupes gekleurd met Masson's trichroom en semi-kwantitatief geanalyseerd.

De hoeveelheid van fibrose, necrose, resorptie lacunae en de ratio "woven/lamellar" bot waren toegenomen bij de hogere bestralingsdoses en aan de zijde met de chirurgische interventie. Ook de wand van de arteria alveolaris inferior was veranderd door de bestraling. De diameter van de arteria alveolaris inferior was afgenomen, en de tunica intima en de tunica media-intima dikte waren toegenomen afhankelijk van de bestralingsdosis. De botombouwsnelheid nam af bij toenemende bestralingsdosis. Chirurgische interventie verhoogde de lokale botombouwsnelheid. Geconcludeerd kan worden dat zowel de bestraling alsook de chirurgische interventie veranderingen van de botstructuur en vaatwand geven. Dit kan leiden tot een chronische hypoxie in het mandibulair bot en daarmee bijdragen aan het ontstaan van ORN.

In Hoofdstuk 8 worden de bevindingen van deze studies nader bediscussieerd. Een afname van doorbloeding en botmineraaldichtheid bij een toenemende bestralingsdosis werden zowel histologisch als met experimentele methoden vastgesteld. Echter is de botmineraaldichtheidsmetingen en de doorbloedingsmetingen met LDF niet geschikt als diagnosticum voor bot veranderingen na bestraling of voor ORN.

Beeldvorming speelt een belangrijke rol in het stellen van de diagnose ORN. Corticale veranderingen en destructie zijn het best zichtbaar met CT-scan, veranderingen van het beenmerg daarentegen zijn het beste zichtbaar met MR-scan. CT- en MR-scans diagnosticeerden zowel de klinische ORN dieren alsook een preklinische ORN welke histologisch bevestigd werd. De sequentie T2-SPIR gaf alleen gemengde signaalintensiteit bij de klinische ORN gevallen, dit benadrukt het nut van het gebruik van deze sequentie bij de diagnostiek van ORN.

$\mathrm{Bij}$ toenemende bestralingsdoses was er een toenemende mate van beenmerg oedeem zichtbaar met MR-scan. Deze toename van beenmerg oedeem kon ook met DE-CT afgeleide VNCa beelden worden zichtbaar gemaakt. Dit biedt de mogelijkheid om met 
één radiologische modaliteit beeldvorming voor de diagnostiek van ORN en bestralingsveranderingen van de mandibula te verrichten.

De pathofysiologie van botschade na bestraling van mandibula welke leidt tot ORN bestaat uit drie componenten: allereerst vasculaire fibrosering welke leidt tot een verminderde doorbloeding, ten tweede een afname van de botombouwsnelheid en verstoorde botarchitectuur en ten derde een ontregelde osteoclasten activiteit. Wij denken dat als de fragiele balans van deze processen verloren gaat en hypoxie daarbij onder een bepaalde grens zakt, ORN zal ontstaan.

De term osteoradionecrose zou derhalve in bestralingsgeïnduceerde osteomyelitis moeten worden gewijzigd, omdat dit beter het onderliggende pathophysiologische proces beschrijft.

\section{VALORISATIE}

Door de variabiliteit in het ontstaan en presentatie alsook de variabiliteit in het spontane beloop van ORN van de mandibula, is het bestuderen van klinische ORN zeer moeizaam. Het bestuderen van de effectiviteit van de behandeling van ORN wordt ook gehinderd door de wisselende uitkomsten van de behandeling van ORN, gecombineerd met de variabiliteit van ORN.

Om de pathophysiologische omstandigheden, welke leiden tot het ontstaan van ORN te bestuderen, hebben we een groot diermodel ontwikkeld. In dit model konden we onder gecontroleerde omstandigheden met toenemende bestralingsdoses de pathophysiologische processen vergelijkbaar met de klinische situatie bestuderen. Door de toepassing van verschillende radiologische modaliteiten maakt de bevindingen in ons diermodel van grote waarde en maakt ook de vertaling van de resultaten naar de klinische situatie mogelijk.

Toekomstige studies zouden patiënt specifieke risicofactoren op het ontwikkelen van ORN moeten bestuderen en de mogelijkheden van de medicamenteuze therapie van ORN moeten exploreren. Ook het verbeteren en klinisch valideren van de toepassing van DE-CT afgeleide beelden voor de detectie van beenmerg oedeem bij vroege of preklinische ORN is een onderwerp voor toekomstige studies. 



\section{Other publications}


Survival after curative surgical treatment for primary oral squamous cell carcinoma. Bloebaum M, Poort L, Böckmann R, Kessler P. J Craniomaxillofac Surg. 2014; 42(8): 1572-6.

Definition of quality indicators in microsurgery in head and neck reconstruction based on a 5-year follow-up without a loss. Kessler P, Poort L, Böckmann R, Lethaus B. J Craniomaxillofac Surg. 2013; 41(1):2-6.

Qualitative bone $\mathrm{CT}$ as a tool to assess vascularization in irradiated bone: an animal study. Lethaus B, Poort L, Yamauchi K, Kloss-Brandstätter A, Böckmann R, Kessler P. Clin Oral Implants Res. 2013; 24(7): 746-9.

Additive manufacturing for microvascular reconstruction of the mandible in 20 patients. Lethaus B, Poort L, Böckman R, Smeets R, Tolba R, Kessler P. J Craniomaxillofac Surg. 2012; 40(1): 43-6.

Reconstruction of a maxillary defect with a fibula graft and titanium mesh using CAD/CAM techniques. Lethaus B, Kessler P, Böckman R, Poort LJ, Tolba R. Head \& face medicine 2010; 19(6):16.

Sensory testing of inferior alveolar nerve injuries: a review of methods used in prospective studies. L.J. Poort, J.W. van Neck, K.G.H. van der Wal. J Oral Maxillofac Surg 2009; 67(2): 292-300.

Open-wond behandeling ter preventie van wondinfectie na abdominale chirurgie in Nederland. L.J. Poort, R.W. Kleinnagelvoort, M. van der Elst, L.P. Stassen. Ned Tijdschr v Heelkd 2006; 15(6): 177-183.

Surgically assisted rapid maxillary expansion (SARME): a review of the literature. M.J. Koudstaal, L.J. Poort, K.G.H. van der Wal, E.B. Wolvius, B. Prahl-Andersen and A.J.M. Schulten. Int J Oral Maxillofac Surg 2005; 34(7):709-14.

Evaluatie van supra-umbilicale versus rechterbovenbuiksincisie bij pyloromyotomie vanwege pylorushypertrofie. E. van den Berg, L.J. Poort, J.W.B. Peeters, R.R. Postema. Ned Tijdschr v Heelkd 2004; 13(5): 126-129. 


\section{Curriculum Vitae}

Lucas Juliën Poort was born on October $22^{\text {nd }} 1975$ in Rotterdam, the Netherlands. He attended high school (VWO) at the Comenius College in Capelle aan den IJssel and graduated in 2001. He studied dentistry at the Free University of Brussels and graduated in 2007. From 2008 until 2013 he specialised in Maxillofacial Surgery at the department of Cranio- and Maxillofacial Surgery at the Maastricht University Medical Centre training under supervision of Professor Kessler. He is presently working as a Maxillofacial surgeon in the Zuyderland Medical Centre in Heerlen and Sittard, and as researcher in the department of Cranio- and Maxillofacial Surgery at the Maastricht University Medical Centre.

Lucas Juliën Poort is geboren op 22 oktober 1975 te Rotterdam. Hij behaalde het VWO diploma aan het Comenius College in Capelle aan den IJssel in 1994. Hij studeerde medicijnen aan de Erasmus Universiteit Rotterdam en behaalde in 2001 het artsexamen. Hij studeerde tandheelkunde aan de Vrije Universiteit van Brussel en ronde deze studie in 2007 af. Van 2008 tot 2013 specialiseerde hij zich in de Mondziekten, kaak- en aangezichtschirurgie aan de gelijknamige afdeling van het Maastricht Universitair Medisch Centrum onder leiding van Professor Kessler. Momenteel werkt hij als specialist Mondziekten, kaak- en aangezichtschirurgie in de maatschap van het Zuyderland ziekenhuis in Heerlen en Sittard. Daarnaast is hij in deeltijd verbonden als onderzoeker aan de afdeling Mondziekten, kaak- en aangezichtschirurgie van het Maastricht Universitair Medisch Centrum. 

Dankwoord 
Dit proefschrift was niet tot stand gekomen zonder de hulp van veel mensen.

Mijn dank aan professor Kessler voor de grote mate van vrijheid om dit onderwerp verder uit te werken en invulling te geven aan de dierproef en het uitwerken van de data. U gaf mij vrijheid maar ook adviezen en steun in de verschillende stadia van het onderzoek.

Mijn co-promotoren, Linda en Frank, hebben een grote en essentiële bijdrage geleverd aan de totstandkoming van dit proefschrift. leder met expertise welke onmisbaar was bij de interpretatie van de uitkomsten van dit onderzoek. Jullie beider snelle reacties op de gestuurde stukken hebben er voor gezorgd dat het proefschrift met deze snelheid is gemaakt.

Afdeling radiotherapie, velen van jullie hebben tot ver na kantooruren gewerkt: Marlies, Rene, Huub, Hans, Kim, Jeroen, Nick, Hilde, Patrick, Jos de Jong en professor Lambin.

Afdeling radiologie, ook steeds bereid tot vervaardigen van scans op late tijdstippen: Jeroen, Ralph, Henk, Roland, Irene, Thea.

CPV: Joyce, Petra, Ivo, Sanne en andere verzorgenden. Ik bewonder jullie toewijding voor het werk.

Afdeling Fysiologie voor het gebruik van de transport module.

Afdeling Orthopedie, Tim, Pieter, Jim, Chris, Marjolein, Alex, Don, Andy voor hulp bij opstellen van de proef, bereiden van de fluorescentie, vervaardigen van de coupes.

Ruud voor de zeer waardevolle ondersteuning en statistische bewerkingen.

WESP studenten Monique, Lisette, Koen, Charlotte, en Hein. Zonder jullie hulp en inzet was het niet gelukt.

Special thanks to Ken for your support of my project.

Afdeling Anatomie voor het gebruik van de microscoop.

Speciale dank voor Henk Verdonck voor al het werk wat jij vooraf gedaan hebt voor jouw proefschrift, wat ten grondslag ligt aan dit werk. Door de bijzondere zoektocht door jou werkkamer kwam ik stap voor stap dichter bij de antwoorden op tal van mijn vragen.

Frans de Beer voor het gebruik van de motoren en advies ter aanvulling op het werk van Henk Verdonck. 
Alle medewerkers van de afdeling Mondziekten, Kaak- en Aangezichtschirurgie Maastricht die mij in veel opzichten gesteund hebben tijdens de proef.

De maatschap Mondziekten, Kaak- en Aangezichtschirurgie van het Elkerliek ziekenhuis voor de mogelijkheid om ook tijdens mijn perifere stage aan mijn onderzoek te werken.

De maatschap Mondziekten, Kaak- en Aangezichtschirurgie van het Zuyderland ziekenhuis: zonder jullie steun had ik het niet zo snel kunnen afronden.

Thea, Pieter en Clements van BioComp voor de financiële en materiële ondersteuning van de proef.

Dank voor de geweldige steun van Mariël, van begin tot het eind, zonder haar was het zeker niet gelukt en was ik er ook niet aan begonnen.

Alle mensen die ik onverhoopt niet heb genoemd: veel dank! 


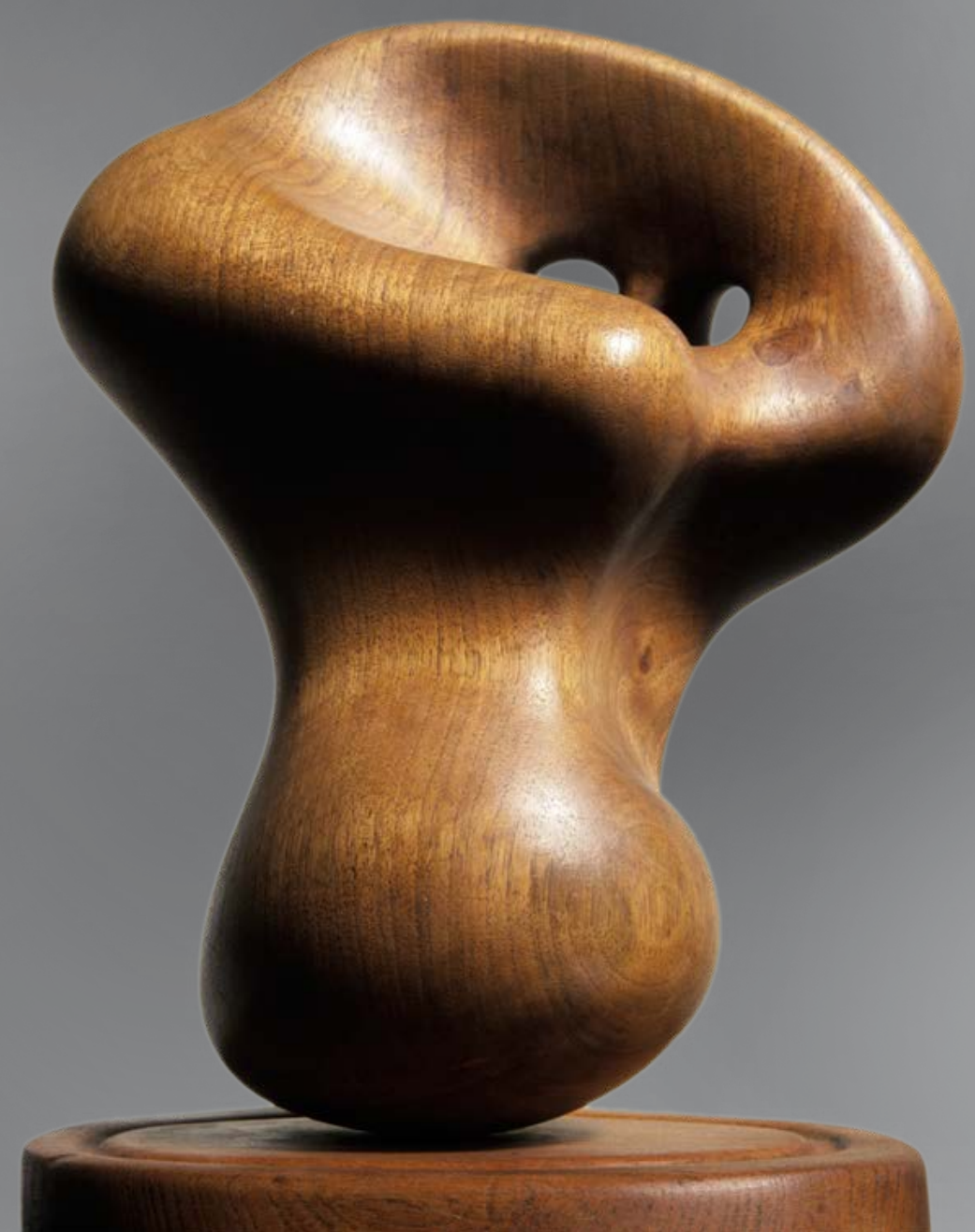

